\title{
Coloring ordinals by reals
}

\author{
by \\ Jörg Brendle (Kobe) and Sakaé Fuchino (Kasugai)
}

\begin{abstract}
We study combinatorial principles we call the Homogeneity Principle $\operatorname{HP}(\kappa)$ and the Injectivity Principle $\operatorname{IP}(\kappa, \lambda)$ for regular $\kappa>\aleph_{1}$ and $\lambda \leq \kappa$ which are formulated in terms of coloring the ordinals $<\kappa$ by reals.

These principles are strengthenings of $\mathrm{C}^{\mathrm{S}}(\kappa)$ and $\mathrm{F}^{\mathrm{S}}(\kappa)$ of I. Juhász, L. Soukup and Z. Szentmiklóssy. Generalizing their results, we show e.g. that $\operatorname{IP}\left(\aleph_{2}, \aleph_{1}\right.$ ) (hence also $\operatorname{IP}\left(\aleph_{2}, \aleph_{2}\right)$ as well as $\left.\mathrm{HP}\left(\aleph_{2}\right)\right)$ holds in a generic extension of a model of $\mathrm{CH}$ by Cohen forcing, and $\operatorname{IP}\left(\aleph_{2}, \aleph_{2}\right)$ (hence also $\operatorname{HP}\left(\aleph_{2}\right)$ ) holds in a generic extension by countable support side-by-side product of Sacks or Prikry-Silver forcing (Corollary 4.8). We also show that the latter result is optimal (Theorem 5.2).

Relations between these principles and their influence on the values of the variations $\mathfrak{b}^{\uparrow}, \mathfrak{b}^{h}, \mathfrak{b}^{*}, \mathfrak{d} \mathfrak{o}$ of the bounding number $\mathfrak{b}$ are studied.

One of the consequences of $\operatorname{HP}(\kappa)$ besides $\mathrm{C}^{\mathrm{s}}(\kappa)$ is that there is no projective wellordering of length $\kappa$ on any subset of ${ }^{\omega} \omega$. We construct a model in which there is no projective well-ordering of length $\omega_{2}$ on any subset of ${ }^{\omega} \omega\left(\mathfrak{d o}=\aleph_{1}\right.$ in our terminology) while $\mathfrak{b}^{*}=\aleph_{2}$ (Theorem 6.4).
\end{abstract}

1. Introduction. The Cohen model which is obtained by adding at least $\aleph_{2}$ Cohen reals over a model of GCH was the first and simplest model for the negation of $\mathrm{CH}$, and it is still one of the most important. A plethora of statements have been shown to be consistent with ZFC by adjoining Cohen reals, and it is therefore natural to look for axioms which hold in the Cohen model and from which many such statements can be decided, that is, axioms which capture as much as possible of the combinatorial structure of the Cohen extension. Something similar has been done for the iterated Sacks model by Ciesielski and Pawlikowski who devised the Covering Property Axiom CPA [2].

2000 Mathematics Subject Classification: 03E05, 03E17, 03E35, 03E65.

Key words and phrases: Homogeneity Principle, Injectivity Principle, bounding number, projective well-ordering, Cohen forcing, Brendle-LaBerge forcing, Prikry-Silver forcing.

The first author is partially supported by Grant-in-Aid for Scientific Research (C) 17540116, Japan Society for the Promotion of Science. The second author is partially supported by Chubu University grant 16IS55A. 
For Cohen models, several such axioms have been proposed in the past. Some of them are homogeneity type statements, that is, they assert that given at least $\omega_{2}$ many reals, many of them "look similar". Examples are the combinatorial principles $\mathrm{C}^{\mathrm{S}}(\kappa), \hat{\mathrm{C}}^{\mathrm{S}}(\kappa)$, and $\mathrm{F}^{\mathrm{S}}(\kappa)$ introduced by I. Juhász, L. Soukup and Z. Szentmiklóssy [13] who showed that these principles hold in Cohen models (see Section 2 below for definitions).

On the other hand, rather different-looking statements have also been investigated in connection with Cohen models, for example, the axiom WFN asserting that $\langle\mathcal{P}(\omega), \subseteq\rangle$ has the weak Freese-Nation property (see [8], [10] and $[6])$. Here a partial ordering $\langle P, \leq\rangle$ has the weak Freese-Nation property if there is a mapping $f: P \rightarrow[P]^{\aleph_{0}}$ such that for all $p, q \in P, p \leq_{P} q$ if and only if there is an $r \in f(p) \cap f(q)$ such that $p \leq_{P} r \leq_{P} q$.

In [8], it is shown that WFN holds in a Cohen model for adding $\aleph_{n}$ Cohen reals for any $n<\omega$. If we start e.g. from $V=L$ then WFN holds even after adding any number of Cohen reals ([10]). In [6], it was shown that WFN implies many of the known combinatorial properties of Cohen models and so it may be seen as an axiomatization of the combinatorial structure of the Cohen extension. Since WFN can be reformulated in terms of elementary submodels, WFN as well as some closely related statements have come to be known as elementary submodel type axioms (see [12] for this).

At first glance it seemed that there would be no connection between these two types of axioms except that they both hold in a Cohen model. Surprisingly enough though, S. Shelah [17] showed that $\mathrm{C}^{\mathrm{s}}\left(\aleph_{2}\right)$ follows from the combinatorial principle he called PRINC, which is a consequence of WFN. The proof can be easily recast to show that WFN implies $\mathrm{C}^{\mathrm{S}}(\kappa)$ for all regular $\kappa>\aleph_{1}$ (see [7] for more details).

In this paper, we introduce some new principles of the homogeneity type, namely, the Homogeneity Principle $\operatorname{HP}(\kappa)$ and the Injectivity Principle $\operatorname{IP}(\kappa, \lambda)$, which are formulated in terms of homogeneity of colorings of ordinals below the cardinal $\kappa$ by reals. We establish that these axioms hold in Cohen models and address the question in which other models these axioms hold as well. It turns out that, in fact, these principles seem to capture a good deal of the combinatorial features of models of set theory obtained by forcing by the side-by-side (finite or countable support) product of copies of a fixed relatively small partial ordering (see Theorem 4.3 and Corollary 4.8).

Though the relation of these principles to WFN is not yet completely clear, our principles imply the principles of I. Juhász, L. Soukup and Z. Szentmiklóssy (Theorem 2.7) and thus can be seen as natural strengthenings of these principles.

Our paper is organized as follows. 
In Section 2, we review the principles $\mathrm{C}^{\mathrm{S}}(\kappa), \hat{\mathrm{C}}^{\mathrm{S}}(\kappa)$ and $\mathrm{F}^{\mathrm{s}}(\kappa)$ of I. Juhász, L. Soukup and Z. Szentmiklóssy, and introduce our principles $\operatorname{HP}(\kappa)$ and $\operatorname{IP}(\kappa, \lambda)$. Some basic facts in ZFC concerning these principles are also proved. In particular, we show that $\mathrm{C}^{\mathrm{S}}(\kappa)$ and $\hat{\mathrm{C}}^{\mathrm{S}}(\kappa)$ follow from $\mathrm{HP}(\kappa)$ (Theorem 2.7), $\mathrm{F}^{\mathrm{S}}(\kappa)$ follows from $\operatorname{IP}\left(\kappa, \aleph_{1}\right)$ (Theorem 2.8), and $\operatorname{HP}(\kappa)$ follows from $\operatorname{IP}(\kappa, \kappa)$ (Theorem 2.9).

After reviewing some cardinal invariants introduced in [7] which are variants of the bounding number $\mathfrak{b}$ and the shrinking number $\mathfrak{b}^{*}$ in [3], we study in Section 3 the effect of the combinatorial principles $\mathrm{C}^{\mathrm{S}}(\kappa), \hat{\mathrm{C}}^{\mathrm{S}}(\kappa)$ and $\operatorname{HP}(\kappa)$ on the values of these cardinal invariants.

In Section 4 we give a forcing construction of models of $\operatorname{IP}(\kappa, \lambda)$ (Theorem 4.3) and its applications (Corollary 4.8). The results in this section improve consistency results in [13].

As a further application of Theorem 4.3 we show in Section 5 the consistency of $\neg \operatorname{IP}\left(\aleph_{2}, \aleph_{1}\right)$ and $\operatorname{IP}\left(\aleph_{2}, \aleph_{2}\right)$.

One of the consequences of $\operatorname{HP}\left(\aleph_{2}\right)$ discussed in Section 3 is that there is no definable well-ordering of length $\omega_{2}$ on any subset of $\omega_{\omega}$ (or $\mathfrak{d o}=\aleph_{1}$ in our notation). Refining a forcing extension of Brendle and LaBerge [1], we prove in Section 6 the consistency of $\mathfrak{d o}=\aleph_{1}$ with $\mathfrak{b}^{*}=\aleph_{2}$ (Theorem 6.4). We also show that the model of $\mathfrak{d o}=\aleph_{1}$ and $\mathfrak{b}^{*}=\aleph_{2}$ we construct in this section satisfies a strong negation of $\mathrm{C}^{\mathrm{S}}\left(\aleph_{2}\right)$.

Section 7 is devoted to the consistency proof of the combinatorial principle used in the proof of Theorem 6.4.

In Section 8, we summarize the consistency results obtained in this paper together with other consistency results established by some previously known constructions. We also discuss some open problems at the end of the section.

2. Combinatorial principles formulated in terms of coloring of ordinals by reals. For any set $X$, let

$$
\begin{aligned}
((X))^{n} & =\left\{\vec{x} \in X^{n}: \vec{x} \text { is injective }\right\} \\
((X))^{<\omega} & =\bigcup_{n<\omega}((X))^{n} .
\end{aligned}
$$

Likewise, for any sets $X_{0}, \ldots, X_{n-1}$, let

$$
\left(\left(X_{0}, \ldots, X_{n-1}\right)\right)=\left\{\vec{x} \in X_{0} \times \cdots \times X_{n-1}: \vec{x} \text { is injective }\right\} .
$$

For a cardinal $\kappa$ of uncountable cofinality, the following principle $\mathrm{C}^{\mathrm{s}}(\kappa)$ was introduced by I. Juhász, L. Soukup and Z. Szentmiklóssy in [13]. 
$\mathrm{C}^{\mathrm{S}}(\kappa)$ : For any matrix $\left\langle a_{\alpha, n}: \alpha \in \kappa, n \in \omega\right\rangle$ of subsets of $\omega$ and $T \subseteq{ }^{\omega>} \omega$, at least one of the following holds:

(c0) there is a stationary $S \subseteq \kappa$ such that $\bigcap_{n<|t|} a_{\alpha_{n}, t(n)} \neq \emptyset$ for all $t \in T$ and for all $\left\langle\alpha_{0}, \ldots, \alpha_{|t|-1}\right\rangle \in((S))^{<\omega}$;

(c1) there exist $t \in T$ and stationary $S_{0}, \ldots, S_{|t|-1} \subseteq \kappa$ such that $\bigcap_{n<|t|} a_{\alpha_{n}, t(n)}=\emptyset$ for all $\left\langle\alpha_{0}, \ldots, \alpha_{|t|-1}\right\rangle \in\left(\left(S_{0}, \ldots, S_{|t|-1}\right)\right)$.

For any cardinal $\kappa$ of uncountable cofinality it is easy to see that $\mathrm{C}^{\mathrm{S}}(\kappa)$ holds if and only if $\mathrm{C}^{\mathrm{S}}(\operatorname{cf} \kappa)$ holds. Thus it is enough to consider $\mathrm{C}^{\mathrm{S}}(\kappa)$ for regular uncountable $\kappa$. The corresponding assertion is also true for other combinatorial principles we are going to introduce in this section. Hence, in the rest of this section, we shall assume that $\kappa$ is a regular uncountable cardinal unless mentioned otherwise.

The combinatorial principle $\hat{\mathrm{C}}^{\mathrm{S}}(\kappa)$, a sort of dual of the principle $\mathrm{C}^{\mathrm{S}}(\kappa)$, is also considered in [13]:

$\hat{\mathrm{C}}^{\mathrm{S}}(\kappa)$ : For any $T \subseteq \omega^{<\omega}$ and any matrix $\left\langle a_{\alpha, n}: \alpha<\kappa, n \in \omega\right\rangle$ of subsets of $\omega$, at least one of the following holds:

(c)0) there is a stationary $S \subseteq \kappa$ such that $\left|\bigcap_{n<|t|} a_{\alpha_{n}, t(n)}\right|<\aleph_{0}$ for all $t \in T$ and for all $\left\langle\alpha_{0}, \ldots, \alpha_{|t|-1}\right\rangle \in((S))^{|t|}$;

(c)1) there exist $t \in T$ and stationary $S_{0}, \ldots, S_{|t|-1} \subseteq \kappa$ such that $\left|\bigcap_{n<|t|} a_{\alpha_{n}, t(n)}\right|=\aleph_{0}$ for every $\left\langle\alpha_{0}, \ldots, \alpha_{|t|-1}\right\rangle \in$ $\left(\left(S_{0}, \ldots, S_{|t|-1}\right)\right)$.

The following is easily seen:

Lemma 2.1 (I. Juhász, L. Soukup and Z. Szentmiklóssy [13]).

(a) Neither $\mathrm{C}^{\mathrm{S}}\left(\aleph_{1}\right)$ nor $\hat{\mathrm{C}}^{\mathrm{S}}\left(\aleph_{1}\right)$ holds.

(b) $\mathrm{C}^{\mathrm{S}}(\kappa)$ and $\hat{\mathrm{C}}^{\mathrm{S}}(\kappa)$ hold for any regular $\kappa>2^{\aleph_{0}}$.

Let us call a subset $A$ of $\mathcal{H}\left(\aleph_{1}\right)$ definable if there are a formula $\varphi$ and $a \in \mathcal{H}\left(\aleph_{1}\right)$ such that $A=\left\{x \in \mathcal{H}\left(\aleph_{1}\right):\left\langle\mathcal{H}\left(\aleph_{1}\right), \in\right\rangle \models \varphi(x, a)\right\}$. Note that for any $n \in \omega, A \subseteq \mathbb{R}^{n}$ is projective if and only if it is definable in our sense. Note also that since all elements of $\mathcal{H}\left(\aleph_{1}\right)$ can be coded by elements of $\omega_{\omega}$ we may assume that $a$ as above is an element of ${ }^{\omega_{\omega}} \omega$.

In Theorem 2.7, we show that the following Homogeneity Principle $\mathrm{HP}(\kappa)$ implies both of $\mathrm{C}^{\mathrm{S}}(\kappa)$ and $\hat{\mathrm{C}}^{\mathrm{S}}(\kappa)$.

$\operatorname{HP}(\kappa):$ For any $f: \kappa \rightarrow \mathcal{P}(\omega)$ and any definable $A \subseteq((\mathcal{P}(\omega)))^{<\omega}$, at least one of the following holds:

(h0) there is a stationary $S \subseteq \kappa$ such that $\left(\left(f^{\prime \prime} S\right)\right)^{<\omega} \backslash\{\emptyset\} \subseteq A$;

(h1) there are $k \in \omega \backslash 1$ and stationary $S_{0}, \ldots, S_{k-1} \subseteq \kappa$ such that $\left(\left(f^{\prime \prime} S_{0}, \ldots, f^{\prime \prime} S_{k-1}\right)\right) \cap A=\emptyset$. 
Note that we obtain an assertion equivalent to $\operatorname{HP}(\kappa)$ if $\mathcal{P}(\omega)$ in the definition of $\operatorname{HP}(\kappa)$ is replaced by $\mathbb{R},{ }^{\omega} \omega,(\mathcal{P}(\omega))^{n}$ or $\left({ }^{\omega} \omega\right)^{n}$ etc., since these spaces can be coded as definable subsets of $\mathcal{P}(\omega)$ and vice versa.

As for $\mathrm{C}^{\mathrm{S}}(\kappa)$ (and $\hat{\mathrm{C}}^{\mathrm{S}}(\kappa)$ ), it is enough to consider $\mathrm{HP}(\kappa)$ for regular $\kappa$. Lemma 2.1 is also true for $\operatorname{HP}(\kappa)$ :

LEMMA 2.2.

(a) $\operatorname{HP}\left(\aleph_{1}\right)$ does not hold.

(b) $\operatorname{HP}(\kappa)$ holds for any regular $\kappa>2^{\aleph_{0}}$.

Proof. (a) This follows from Lemma 2.1 and Theorem 2.7.

(b) Let $\kappa>2^{\aleph_{0}}$ be a regular cardinal. Suppose that $f: \kappa \rightarrow \mathcal{P}(\omega)$ and $A$ are as in the definition of $\operatorname{HP}(\kappa)$. Then there is a stationary $S \subseteq \kappa$ such that $f\lceil S$ is constant. If (h0) in the definition of $\operatorname{HP}(\kappa)$ does not hold then we must have $\left(\left(f^{\prime \prime} S\right)\right)^{1} \cap A=\emptyset$ since $\left(\left(f^{\prime \prime} S\right)\right)^{n}=\emptyset$ for all $n>1$. Hence (h1) holds with $n=1$ and $S_{0}=S$.

(Lemma 2.2)

The following combinatorial principle $\mathrm{F}^{\mathrm{S}}(\kappa)$ is also introduced in [13]:

$\mathrm{F}^{\mathrm{S}}(\kappa):$ For any $T \subseteq \omega^{<\omega}$ and any matrix $\left\langle a_{\alpha, n}: \alpha<\kappa, n \in \omega\right\rangle$ of subsets of $\omega$, at least one of the following holds:

(f0) there is a stationary $S \subseteq \kappa$ such that

$$
\mid\left\{\bigcap_{n<|t|} a_{\alpha_{n}, t(n)}: t \in T \text { and }\left\langle\alpha_{0}, \ldots, \alpha_{|t|-1}\right\rangle \in((S))^{|t|}\right\} \mid \leq \aleph_{0}
$$

(f1) there are $t \in T$ and stationary $S_{0}, \ldots, S_{|t|-1} \subseteq \kappa$ such that for every $\left\langle\alpha_{0}, \ldots, \alpha_{|t|-1}\right\rangle,\left\langle\beta_{0}, \ldots, \beta_{|t|-1}\right\rangle \in\left(\left(S_{0}, \ldots, S_{|t|-1}\right)\right)$, if $\alpha_{n} \neq \beta_{n}$ for all $n<|t|$, then

$$
\bigcap_{n<|t|} a_{\alpha_{n}, t(n)} \neq \bigcap_{n<|t|} a_{\beta_{n}, t(n)} .
$$

Lemma 2.3 (I. Juhász, L. Soukup and Z. Szentmiklóssy [13]).

(a) $\mathrm{F}^{\mathrm{s}}\left(\aleph_{1}\right)$ does not hold.

(b) $\mathrm{F}^{\mathrm{S}}(\kappa)$ holds for every regular $\kappa>2^{\aleph_{0}}$.

(c) $\mathrm{F}^{\mathrm{S}}(\kappa)$ implies $\hat{\mathrm{C}}^{\mathrm{S}}(\kappa)$.

A combinatorial principle in terms of coloring of ordinals by reals corresponding naturally to $\mathrm{F}^{\mathrm{s}}(\kappa)$ might be the following Injectivity Principle $\operatorname{IP}(\kappa, \lambda)$ for cardinals $\kappa$ and $\lambda$ with $\lambda \leq \kappa$ :

$\operatorname{IP}(\kappa, \lambda):$ For any $f: \kappa \rightarrow \mathcal{P}(\omega)$ and definable $g:((\mathcal{P}(\omega)))^{<\omega} \rightarrow \mathcal{P}(\omega)$, at least one of the following holds: 
(i0) there is a stationary $S \subseteq \kappa$ such that $\left|g^{\prime \prime}\left(\left(f^{\prime \prime} S\right)\right)^{n}\right|<\lambda$ for every $n \in \omega$

(i1) there are $k \in \omega \backslash 1$ and stationary $S_{0}, \ldots, S_{k-1} \subseteq \kappa$ such that for any $\left\langle x_{0}, \ldots, x_{k-1}\right\rangle$ and $\left\langle y_{0}, \ldots, y_{k-1}\right\rangle$ in $\left(\left(f^{\prime \prime} S_{0}, \ldots, f^{\prime \prime} S_{k-1}\right)\right)$, if $x_{n} \neq y_{n}$ for all $n<k$, then we have $g\left(x_{0}, \ldots, x_{k-1}\right) \neq g\left(y_{0}, \ldots, y_{k-1}\right)$.

Again, $\mathcal{P}(\omega)$ in the definition of $\operatorname{IP}(\kappa, \lambda)$ above may be replaced by $\mathbb{R},{ }^{\omega} \omega$, $(\mathcal{P}(\omega))^{n}$ or $\left({ }^{\omega} \omega\right)^{n}$ etc. to obtain an equivalent assertion.

LEMMA 2.4 .

(a) For $\lambda \leq \lambda^{\prime} \leq \kappa, \operatorname{IP}(\kappa, \lambda)$ implies $\operatorname{IP}\left(\kappa, \lambda^{\prime}\right)$.

(b) $\operatorname{IP}\left(\aleph_{1}, \aleph_{1}\right)$ does not hold.

Proof. (a) Immediate from the definition.

(b) By Lemma 2.2(a) and Theorem 2.9. - (Lemma 2.4)

$\operatorname{IP}\left(\kappa, \aleph_{0}\right)$ for a regular cardinal $\kappa$ is equivalent to the cardinal inequality $2^{\aleph_{0}}<\kappa$.

Proposition 2.5. For a regular cardinal $\kappa$ the following are equivalent:
(a) $\operatorname{IP}\left(\kappa, \aleph_{0}\right)$ holds;
(b) $2^{\aleph_{0}}<\kappa$;
(c) $\operatorname{IP}(\kappa, 2)$ holds.

Proof. (a) $\Rightarrow$ (b): Suppose that $2^{\aleph_{0}} \geq \kappa$. We show that $\operatorname{IP}\left(\kappa, \aleph_{0}\right)$ does not hold. Let $f: \kappa \rightarrow \mathcal{P}(\omega)$ be any injective mapping and $g:((\mathcal{P}(\omega)))^{<\omega} \rightarrow \mathcal{P}(\omega)$ be defined by $g(\emptyset)=\emptyset, g(\langle x\rangle)=\emptyset$ for all $x \in \mathcal{P}(\omega)$ and

$$
g\left(\left\langle x_{0}, \ldots, x_{n-1}\right\rangle\right)=\min \left\{m \in \omega: m \in x_{0} \leftrightarrow m \in x_{1}\right\}
$$

for $\left\langle x_{0}, \ldots, x_{n-1}\right\rangle \in((\mathcal{P}(\omega)))^{n}$ with $n \geq 2$. Let $S$ be any stationary subset of $\kappa$. Then $\left|g^{\prime \prime}\left(\left(f^{\prime \prime} S\right)\right)^{2}\right| \geq \aleph_{0}$ : Suppose not and let $k \in \omega$ be such that $g^{\prime \prime}\left(\left(f^{\prime \prime} S\right)\right)^{2} \subseteq k$. Since $\mathcal{P}(k+1)$ is finite, there are $\alpha, \beta \in S, \alpha \neq \beta$, such that $f(\alpha) \cap(k+1)=f(\beta) \cap(k+1)$. But then, by definition of $g$, it follows that $g(\langle f(\alpha), f(\beta)\rangle)>k$. This is a contradiction.

Thus (i0) does not hold for these $f$ and $g$. On the other hand, for arbitrary stationary subsets $S_{0}, \ldots, S_{n-1}$ of $\kappa$, as there are only countably many values of $g$, we can find $\left\langle x_{0}, \ldots, x_{n-1}\right\rangle,\left\langle y_{0}, \ldots, y_{n-1}\right\rangle \in\left(\left(f^{\prime \prime} S_{0}, \ldots, f^{\prime \prime} S_{n-1}\right)\right)$ such that $x_{i} \neq y_{i}$ for all $i<n$ and $g\left(\left\langle x_{0}, \ldots, x_{n-1}\right\rangle\right)=g\left(\left\langle y_{0}, \ldots, y_{n-1}\right\rangle\right)$. Thus (i1) does not hold either.

(b) $\Rightarrow\left(\right.$ c): Suppose $2^{\aleph_{0}}<\kappa$. For $f: \kappa \rightarrow \mathcal{P}(\omega)$ and $g:((\mathcal{P}(\omega)))^{<\omega} \rightarrow \mathcal{P}(\omega)$ as in the definition of $\operatorname{IP}(\kappa, 2)$, there is a stationary $S \subseteq \kappa$ such that $f$ is constant on $S$. This $S$ witnesses that (i0) holds.

(c) $\Rightarrow(\mathrm{a})$ : This follows from Lemma 2.4(a). - (Proposition 2.5)

Corollary 2.6. $\operatorname{IP}\left(\aleph_{2}, \aleph_{0}\right)$ is equivalent to $\mathrm{CH}$.

$\operatorname{IP}\left(\aleph_{2}, \aleph_{1}\right)$ and $\operatorname{IP}\left(\aleph_{2}, \aleph_{2}\right)$ are thus the first two non-trivial instances of $\operatorname{IP}(\kappa, \lambda)$. 
For $\kappa \geq \aleph_{2}$, the principles introduced in this section and some other principles discussed in [7] can be put together in the following diagram:

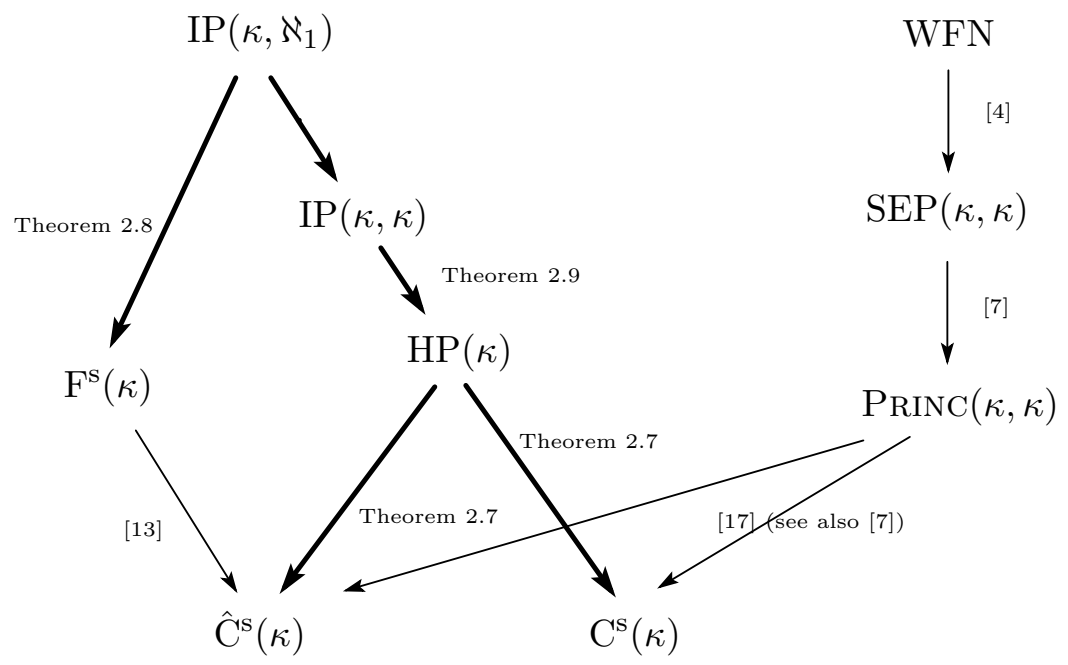

Fig. 1

In the rest of the section, we shall prove the implications indicated by the thick arrows in Fig. 1.

THEOREM 2.7. For a regular cardinal $\kappa, \operatorname{HP}(\kappa)$ implies both $\mathrm{C}^{\mathrm{S}}(\kappa)$ and $\hat{\mathrm{C}}^{\mathrm{S}}(\kappa)$.

Proof. We prove that $\mathrm{HP}(\kappa)$ implies $\mathrm{C}^{\mathrm{S}}(\kappa)$. The other implication can be proved similarly.

By Lemma 2.1(b), we may assume that $\kappa \leq 2^{\aleph_{0}}$. Let $\left\langle t_{i}: i \in \omega\right\rangle$ be an enumeration of ${ }^{\omega>} \omega$ such that

$$
\left|t_{i}\right| \leq i \quad \text { for all } i<\omega
$$

and let $\iota: \mathcal{P}(\omega) \rightarrow \mathcal{P}(\omega)^{\omega}$ be a definable bijection. For each $x \in \mathcal{P}(\omega)$ and $i<\omega$, let $(x)_{i}$ denote the $i$ th component of $\iota(x)$.

Suppose that $T \subseteq{ }^{\omega}{ }^{\omega} \omega$ and $\mathcal{A}=\left\langle a_{\alpha, n}: \alpha<\kappa, n \in \omega\right\rangle$ is a matrix of subsets of $\omega$. We show that either (c0) or (c1) holds for these $\mathcal{A}$ and $T$.

Let $g: \kappa \rightarrow \mathcal{P}(\omega)$ be a fixed injective mapping which exists by $\kappa \leq 2^{\aleph_{0}}$. Let $f: \kappa \rightarrow \mathcal{P}(\omega)$ be defined by

$$
f(\alpha)=\iota^{-1}\left(\left\langle a_{\alpha, n}^{\prime}: n \in \omega\right\rangle\right)
$$

where

$$
a_{\alpha, n}^{\prime}= \begin{cases}g(\alpha) & \text { if } n=0, \\ a_{\alpha, n-1} & \text { otherwise }\end{cases}
$$


Note that $f$ is injective by the "if $n=0$ " clause of (2.6). For $i<\omega$, let

$$
\begin{aligned}
A_{i}^{*} & = \begin{cases}\left\{\left\langle x_{0}, \ldots, x_{i-1}\right\rangle \in((\mathcal{P}(\omega)))^{i}: \bigcap_{n<\left|t_{i}\right|}\left(x_{n}\right)_{t_{i}(n)+1} \neq \emptyset\right\} & \text { if } t_{i} \in T, \\
((\mathcal{P}(\omega)))^{i} & \text { otherwise, }\end{cases} \\
A & =\bigcup_{i<\omega} A_{i}^{*} .
\end{aligned}
$$

It is easy to see that $A$ is definable by noting that $T \in \mathcal{H}\left(\aleph_{1}\right)$ and hence $T$ can be used as a parameter in the definition of $A$. $\operatorname{By} \operatorname{HP}(\kappa)$, we have either (h0) or (h1) for these $A$ and $f$.

Assume first that (h0) holds. Then there is a stationary $S \subseteq \kappa$ such that $\left(\left(f^{\prime \prime} S\right)\right)^{<\omega} \backslash\{\emptyset\} \subseteq A$. We show that this $S$ witnesses (c0) for $T$ and $\mathcal{A}$ : for $t \in T$, let $i \in \omega$ be such that $t=t_{i}$. By $(2.4)$, we have $|t| \leq i$. For $s \in((S))^{|t|}$, let $s^{\prime} \in((S))^{i}$ be an end-extension of $s$. Then $\left\langle f\left(s^{\prime}(0)\right), \ldots, f\left(s^{\prime}(i-1)\right)\right\rangle \in$ $\left(\left(f^{\prime \prime} S\right)\right)^{i}$ since $f$ is injective. Hence $\left\langle f\left(s^{\prime}(0)\right), \ldots, f\left(s^{\prime}(i-1)\right)\right\rangle \in A_{i}^{*}$ by the assumption on $S$. By (2.7), we have

$$
\emptyset \neq \bigcap_{n<\left|t_{i}\right|}\left(f\left(s^{\prime}(n)\right)\right)_{t_{i}(n)+1}=\bigcap_{n<\left|t_{i}\right|} a_{s^{\prime}(n), t_{i}(n)+1}^{\prime}=\bigcap_{n<|t|} a_{s(n), t(n)} .
$$

Thus $T$ and $\mathcal{A}$ satisfy $(\mathrm{c} 0)$.

Assume now that (h1) holds. In this case, there are $i \in \omega$ and stationary $S_{0}, \ldots, S_{i-1} \subseteq \kappa$ such that

$$
\left(\left(f^{\prime \prime} S_{0}, \ldots, f^{\prime \prime} S_{i-1}\right)\right) \subseteq((\mathcal{P}(\omega)))^{i} \backslash A_{i}^{*} .
$$

Let $t=t_{i}$. Then $t \in T$ by (2.9) and the "otherwise" clause of (2.7). For $s \in\left(\left(S_{0}, \ldots, S_{|t|-1}\right)\right)$, let $s^{\prime} \in\left(\left(S_{0}, \ldots, S_{i-1}\right)\right)$ be an end-extension of $s$. Then we have $\left\langle f\left(s^{\prime}(0)\right), \ldots, f\left(s^{\prime}(i-1)\right)\right\rangle \in\left(\left(f^{\prime \prime} S_{0}, \ldots, f^{\prime \prime} S_{i-1}\right)\right)$. It follows that

$$
\left\langle f\left(s^{\prime}(0)\right), \ldots, f\left(s^{\prime}(i-1)\right)\right\rangle \in((\mathcal{P}(\omega)))^{i} \backslash A_{i}^{*}
$$

by (2.9). Hence, by (2.7), we have

$$
\emptyset=\bigcap_{n<|t|}\left(f\left(s^{\prime}(n)\right)\right)_{t(n)+1}=\bigcap_{n<\left|t_{i}\right|} a_{s^{\prime}(n), t(n)+1}^{\prime}=\bigcap_{n<|t|} a_{s(n), t(n)} .
$$

Thus, $T$ and $\mathcal{A}$ satisfy (c1) in this case.

The proof of $\hat{\mathrm{C}}^{\mathrm{S}}(\kappa)$ from $\operatorname{HP}(\kappa)$ is exactly like the proof above with (2.7) replaced by

$(2.7)^{\prime} \quad A_{i}^{*}=\left\{\begin{array}{rr}\left\{\left\langle x_{0}, \ldots, x_{i-1}\right\rangle \in((\mathcal{P}(\omega)))^{i}:\left|\bigcap_{n<\left|t_{i}\right|}\left(x_{n}\right)_{t_{i}(n)+1}\right|<\aleph_{0}\right\} \\ \\ ((\mathcal{P}(\omega)))^{i} & \text { if } t_{i} \in T,\end{array}\right.$

$\operatorname{HP}(\kappa)$ also imply other variants of $\mathrm{C}^{\mathrm{s}}(\kappa)$. For example, let 
${ }^{*} \mathrm{C}^{\mathrm{S}}(\kappa):$ For any matrix $\left\langle a_{\alpha, n}: \alpha \in \kappa, n \in \omega\right\rangle$ of subsets of $\omega$ and $T \subseteq$ ${ }^{\omega>} \omega$, at least one of the following holds:

$\left({ }^{*} \mathrm{c} 0\right)$ there is a stationary $S \subseteq \kappa$ such that $\bigcap_{n<|t|} a_{\alpha_{n}, t(n)}$ is infinite for all $t \in T$ and all $\left\langle\alpha_{0}, \ldots, \alpha_{|t|-1}\right\rangle \in((S))^{<\omega}$;

$\left({ }^{*} \mathrm{c} 1\right)$ there exist $t \in T$ and stationary $S_{0}, \ldots, S_{|t|-1} \subseteq \kappa$ such that $\bigcap_{n<|t|} a_{\alpha_{n}, t(n)}$ is finite for all $\left\langle\alpha_{0}, \ldots, \alpha_{|t|-1}\right\rangle \in$ $\left(\left(S_{0}, \ldots, S_{|t|-1}\right)\right)$.

It is easy to see by a proof similar to that of Theorem 2.7 that $\mathrm{HP}(\kappa)$ implies ${ }^{*} \mathrm{C}^{\mathrm{S}}(\kappa)$ as well.

The following can also be proved similarly to Theorem 2.7 .

Theorem 2.8. $\operatorname{IP}\left(\kappa, \aleph_{1}\right)$ implies $\mathrm{F}^{\mathrm{S}}(\kappa)$.

Theorem 2.9. $\operatorname{IP}(\kappa, \kappa)$ implies $\operatorname{HP}(\kappa)$.

Proof. Suppose that $A \subseteq((\mathcal{P}(\omega)))^{<\omega}$ is definable and $f: \kappa \rightarrow \mathcal{P}(\omega)$. If $f^{-1}[\{x\}]$ is stationary for some $x \in \mathcal{P}(\omega)$, then either (h0) holds for $S=f^{-1}[\{x\}]$ or (h1) holds for $n=1$ and $S_{0}=f^{-1}[\{x\}]$, depending on whether $x \in A$ or not. Otherwise let $g:((\mathcal{P}(\omega)))^{<\omega} \rightarrow \mathcal{P}(\omega)$ be defined by

$$
\begin{aligned}
& g(\emptyset)=\emptyset ; \\
& g(\langle x\rangle)=\emptyset \quad \text { for all } x \in \mathcal{P}(\omega) ; \\
& g\left(\left\langle x_{0}, \ldots, x_{n-1}, x\right\rangle\right)= \begin{cases}\emptyset & \text { if }\left\langle x_{0}, \ldots, x_{n-1}\right\rangle \in A, \\
x & \text { otherwise }\end{cases}
\end{aligned}
$$

for all $\left\langle x_{0}, \ldots, x_{n-1}, x\right\rangle \in((\mathcal{P}(\omega)))^{n+1}$. If (i0) holds for this $g$ with $S$ as in (i0), then, by (2.12), we must have $g^{\prime \prime}\left(\left(f^{\prime \prime} S\right)\right)^{<\omega}=\{\emptyset\}$. Hence $\left(\left(f^{\prime \prime} S\right)\right)^{<\omega} \backslash\{\emptyset\}$ $\subseteq A$. On the other hand, if (i1) holds for some $n<\omega$ and $S_{0}, \ldots, S_{n-1}$, then we should have $n \geq 2$ by $(2.11)$ and $g\left(\left\langle x_{0}, \ldots, x_{n-2}, x_{n-1}\right\rangle\right)=x_{n-1}$ for all $x_{i} \in f^{\prime \prime} S_{i}, i<n$ by $(2.12)$. It follows that $\left(\left(f^{\prime \prime} S_{0}, \ldots, f^{\prime \prime} S_{n-2}\right)\right) \subseteq$ $((\mathcal{P}(\omega)))^{n-1} \backslash A$ by (2.12). - (Theorem 2.9)

3. The bounding number and its variations. In this section, we show that the combinatorial principles introduced in the last section make some of the cardinal invariants from [7] small.

Adopting the notation of [7], we consider the following spectra of cardinal numbers in connection with a partial ordering $\langle P, \leq\rangle$ (called the unbounded spectrum, hereditary unbounded spectrum and the spectrum of length of $P$ ):

$$
\begin{aligned}
& \mathfrak{S}(P)=\{|X|: X \subseteq P, X \text { is unbounded in } P, \\
&\left.\forall B \in[X]^{<|X|}(B \text { is bounded in } P)\right\}, \\
& \mathfrak{S}^{h}(P)=\{|X|: X \subseteq P, \forall B \subseteq X(B \text { is bounded in } P \leftrightarrow|B|<|X|)\}, \\
& \mathfrak{S}^{\uparrow}(P)=\{\operatorname{cf}(C): C \subseteq P, C \text { is an unbounded chain }\} .
\end{aligned}
$$


Clearly, we have

$$
\mathfrak{S}^{\uparrow}(P) \subseteq \mathfrak{S}^{h}(P) \subseteq \mathfrak{S}(P) .
$$

For $P=\left\langle\omega^{\omega}, \leq^{*}\right\rangle$, we shall simply write $\mathfrak{S}^{\uparrow}, \mathfrak{S}^{h}$ and $\mathfrak{S}$ in place of $\mathfrak{S}^{\uparrow}\left(\left\langle\omega^{\omega} \omega, \leq^{*}\right\rangle\right), \mathfrak{S}^{h}\left(\left\langle\omega \omega, \leq^{*}\right\rangle\right)$ and $\mathfrak{S}\left(\left\langle{ }^{\omega} \omega, \leq^{*}\right\rangle\right)$, respectively.

Recall that the bounding number $\mathfrak{b}$ is defined by

$$
\mathfrak{b}=\min \left\{|X|: X \subseteq{ }^{\omega} \omega \text { is unbounded with respect to } \leq^{*}\right\} .
$$

The variant $\mathfrak{b}^{*}$ of $\mathfrak{b}$ was introduced and studied in [3] and [14] where

$\mathfrak{b}^{*}=\min \left\{\kappa: \forall X \subseteq{ }^{\omega} \omega(X\right.$ is unbounded

$$
\left.\left.\rightarrow \exists X^{\prime} \in[X]^{\leq \kappa}\left(X^{\prime} \text { is unbounded }\right)\right)\right\} .
$$

$\mathfrak{b}$ and $\mathfrak{b}^{*}$ can be characterized in terms of $\mathfrak{S}^{\uparrow}, \mathfrak{S}^{h}$ and $\mathfrak{S}$ as follows:

LEMMA 3.1.

(a) $\mathfrak{b}=\min \mathfrak{S}^{\uparrow}=\min \mathfrak{S}^{h}=\min \mathfrak{S}$.

(b) $\mathfrak{b}^{*}=\sup \mathfrak{S}$.

In analogy to Lemma $3.1(\mathrm{~b})$, let

$$
\mathfrak{b}^{\uparrow}=\sup \mathfrak{S}^{\uparrow}, \quad \mathfrak{b}^{h}=\sup \mathfrak{S}^{h} .
$$

Recall also that the dominating number $\mathfrak{d}$ is defined as

$$
\mathfrak{d}=\min \left\{|X|: X \subseteq{ }^{\omega} \omega, X \text { dominates }{ }^{\omega} \omega\right\} .
$$

By (3.1) and Lemma 3.1, we have

LEMMA 3.2. $\mathfrak{b} \leq \mathfrak{b}^{\uparrow} \leq \mathfrak{b}^{h} \leq \mathfrak{b}^{*} \leq \mathfrak{d}$.

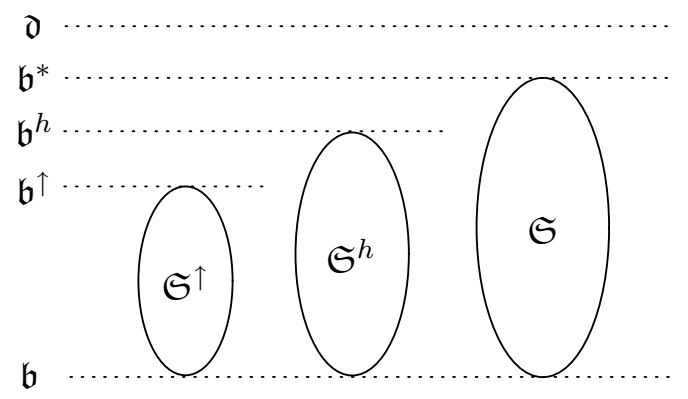

Fig. 2

Let

$$
\begin{aligned}
\mathfrak{D O}=\{\operatorname{cf}(\operatorname{otp}(\langle X, R\lceil X\rangle)): & X \subseteq \omega_{\omega}, R \text { is a definable binary } \\
& \text { relation and } \left.R \cap X^{2} \text { well orders } X\right\}, \\
\mathfrak{d} \mathfrak{o}=\sup \mathfrak{D O} . &
\end{aligned}
$$

By definition, $\mathfrak{S}^{\uparrow} \subseteq \mathfrak{D} \mathfrak{O}$. Hence

LEMMA 3.3. $\mathfrak{b}^{\uparrow} \leq \mathfrak{d} \mathfrak{o}$. 
Lemmas 3.2 and 3.3 may be put together into the following diagram:

$$
\begin{gathered}
\mathfrak{d} \mathfrak{o} \\
\vee \mathrm{I} \\
\mathfrak{b} \leq \mathfrak{b}^{\uparrow} \leq \mathfrak{b}^{h} \leq \mathfrak{b}^{*} \leq \mathfrak{d}
\end{gathered}
$$

Fig. 3

If $\mathfrak{S}^{\uparrow}$ has a maximal element then we have $\mathfrak{b}^{\uparrow}=\max \mathfrak{S}^{\uparrow}$. In that case we shall say that $\mathfrak{b}^{\uparrow}$ is attained. Also we shall say that $\mathfrak{b}^{*}, \mathfrak{b}^{h}$ or $\mathfrak{d o}$ is attained if the corresponding set has a maximal element.

In the following, Reg denotes the class of regular cardinals. The following lemma can be proved similarly to Lemma 3.7(c).

Lemma 3.4 ([7]). $\mathfrak{S}^{h} \cap \operatorname{Reg} \subseteq \mathfrak{D O}$.

Corollary 3.5. If $\mathfrak{S}^{h} \cap \operatorname{Reg}$ is cofinal in $\mathfrak{S}^{h}$ then $\mathfrak{b}^{h} \leq \mathfrak{d} \mathfrak{o}$.

Note that the condition " $\mathfrak{S}^{h} \cap \operatorname{Reg}$ is cofinal in $\mathfrak{S}^{h}$ " holds if $2^{\aleph_{0}}<\aleph_{\omega}$ or if $\mathfrak{b}^{h}$ is regular and attained. Under this condition, we can thus improve the diagram in Fig. 3 to the following:

$$
\begin{gathered}
\mathfrak{d} \mathfrak{o} \\
\vee \mathfrak{l} \\
\mathfrak{b} \leq \mathfrak{b}^{\uparrow} \leq \mathfrak{b}^{h} \leq \mathfrak{b}^{*} \leq \mathfrak{d}
\end{gathered}
$$

Fig. 4

For an ideal $I$ over a set $X, \operatorname{non}(I)$ and $\operatorname{cov}(I)$ denote, as usual, the uniformity and the covering number of $I$, respectively. More exactly

$$
\begin{aligned}
& \operatorname{non}(I)=\min \{|A|: A \in \mathcal{P}(X) \backslash I\}, \\
& \operatorname{cov}(I)=\min \{|\mathcal{A}|: \mathcal{A} \subseteq I, \bigcup \mathcal{A}=X\} .
\end{aligned}
$$

meager and null denote the ideal of meager sets and the ideal of null sets (over $\mathbb{R})$ respectively.

Lemma 3.6. Suppose that $I$ is an ideal over $\mathbb{R}$ with Borel basis. Then $\min \{\operatorname{non}(I), \operatorname{cov}(I)\} \leq \mathfrak{d o}$. In particular,

$\min \{$ non(meager), $\operatorname{cov}($ meager $)\} \leq \mathfrak{d o}, \quad \min \{$ non(null), $\operatorname{cov}($ null $)\} \leq \mathfrak{d} \mathfrak{o}$.

Proof. Suppose that $I \subseteq \mathcal{P}(\mathbb{R})$ is an ideal with a Borel basis and $\kappa=$ $\min \{\operatorname{non}(I), \operatorname{cov}(I)\}$. We can construct inductively a sequence $\left\langle\left\langle f_{\alpha}, g_{\alpha}\right\rangle\right.$ : $\alpha<\kappa\rangle$ such that

$$
f_{\alpha}, g_{\alpha} \in \omega^{\omega} \text { for all } \alpha<\kappa
$$

(3.4) $g_{\alpha}$ codes a Borel set $X_{\alpha} \subseteq \omega_{\omega}$ such that $X_{\alpha} \in I$ and $\left\{f_{\beta}: \beta<\alpha\right\}$ $\subseteq X_{\alpha}$ 


$$
f_{\alpha} \notin \bigcup_{\beta<\alpha} X_{\beta} \text { for all } \alpha<\kappa .
$$

Note that (3.4) is possible by $\kappa \leq \operatorname{non}(I)$, and (3.5) by $\kappa \leq \operatorname{cov}(I)$.

The sequence $\left\langle\left\langle f_{\alpha}, g_{\alpha}\right\rangle: \alpha<\kappa\right\rangle$ is well ordered in order type $\kappa$ by the definable ordering:

$$
\left\langle f^{\prime}, g^{\prime}\right\rangle \leq\langle f, g\rangle \Leftrightarrow f^{\prime} \text { is an element of the Borel set coded by } g .
$$

It follows that $\kappa \leq \mathfrak{d o}$. - (Lemma 3.6)

The following lemma shows the relations of cardinal numbers $\mathfrak{b}, \mathfrak{b}^{\uparrow}, \mathfrak{b}^{h}$, $\mathfrak{d o}$ to the combinatorial principles introduced in Section 2.

LEMMA 3.7.

(a) (I. Juhász, L. Soukup and Z. Szentmiklóssy [13]) If there is $a \leq^{*}$ chain of length $\kappa$ then $\neg \mathrm{C}^{\mathrm{S}}(\kappa)$ and $\neg \hat{\mathrm{C}}^{\mathrm{S}}(\kappa)$. In particular, $\kappa \in \mathfrak{S}^{\uparrow}$ implies $\neg \mathrm{C}^{\mathrm{S}}(\kappa)$ and $\neg \hat{\mathrm{C}}^{\mathrm{S}}(\kappa)$.

(b) $\mathrm{C}^{\mathrm{S}}(\kappa)$ (or $\left.\hat{\mathrm{C}}^{\mathrm{S}}(\kappa)\right)$ implies $\mathfrak{b}^{\uparrow} \leq \kappa$. If $\mathfrak{b}^{\uparrow}$ is attained then $\mathrm{C}^{\mathrm{S}}(\kappa)$ (or $\left.\hat{\mathrm{C}}^{\mathrm{S}}(\kappa)\right)$ implies $\mathfrak{b}^{\uparrow}<\kappa$.

(c) If $\kappa \leq \lambda$ for some $\lambda \in \mathbb{S}^{h}$ with $\mathrm{cf} \lambda \geq \kappa$ then $\neg \mathrm{C}^{\mathrm{S}}(\kappa)$ and $\neg \hat{\mathrm{C}}^{\mathrm{S}}(\kappa)$.

(d) If $\mathfrak{S}^{h} \cap \operatorname{Reg}$ is cofinal in $\mathfrak{S}^{h}$ then $\mathrm{C}^{\mathrm{S}}(\kappa)\left(\right.$ or $\left.\hat{\mathrm{C}}^{\mathrm{S}}(\kappa)\right)$ implies $\mathfrak{b}^{h} \leq \kappa$. If $\mathfrak{b}^{h}$ is regular and attained then $\mathrm{C}^{\mathrm{S}}(\kappa)\left(\right.$ or $\left.\hat{\mathrm{C}}^{\mathrm{S}}(\kappa)\right)$ implies $\mathfrak{b}^{h}<\kappa$.

(e) $\kappa \in \mathfrak{D O}$ implies $\neg \mathrm{HP}(\kappa)$.

(f) $\operatorname{HP}(\kappa)$ implies $\mathfrak{d o} \leq \kappa$. If $\mathfrak{d o}$ is attained then $\operatorname{HP}(\kappa)$ implies $\mathfrak{d o}<\kappa$.

Proof. (a) See [13].

(b) This follows from (a).

(c) Suppose that $\kappa \leq \lambda \in \mathfrak{S}^{h}$ and $\kappa \leq \mathrm{cf} \lambda$. We show $\neg \mathrm{C}^{\mathrm{S}}(\kappa) ; \neg \hat{\mathrm{C}}^{\mathrm{S}}(\kappa)$ can be proved similarly from these assumptions.

Let $X \subseteq \omega_{\omega}$ with $|X|=\lambda$ be as in the definition of $\mathfrak{S}^{h}$. Then we can find $f_{\alpha} \in X$ and $g_{\alpha} \in{ }^{\omega} \omega$ for $\alpha<\kappa$ such that

(3.6) $f_{\alpha} \leq^{*} g_{\beta}$ for all $\alpha<\beta<\kappa$;

(3.7) $f_{\beta} \not^{*} g_{\alpha}^{+}$for all $\alpha \leq \beta<\kappa$ where $g_{\alpha}^{+}$is defined by $g_{\alpha}^{+}(k)=g_{\alpha}(k)+1$ for all $k \in \omega$.

Note that (3.7) is possible since $\operatorname{cf}(|X|) \geq \kappa$.

For $\alpha<\kappa$, let $g_{\alpha, n} \in{ }^{\omega} \omega, n \in \omega$, be such that

$$
\left\{g_{\alpha, n}: n \in \omega\right\}=\left\{g \in{ }^{\omega} \omega: g={ }^{*} g_{\alpha}\right\} .
$$

Let

$$
\begin{aligned}
a_{\alpha, 0} & =\left\{\langle k, l\rangle \in \omega^{2}: l \leq f_{\alpha}(k)\right\}, \\
a_{\alpha, n+1} & =\left\{\langle k, l\rangle \in \omega^{2}: l>g_{\alpha, n}(k)\right\} \quad \text { for all } n \in \omega .
\end{aligned}
$$

We show that $\mathcal{A}=\left\langle a_{\alpha, n}: \alpha \in \kappa, n \in \omega\right\rangle$ with $T=\{\langle 0, n\rangle: n \in \omega \backslash 1\}$ is a counter-example to $\mathrm{C}^{\mathrm{s}}(\kappa)$. 
Suppose first that $S \subseteq \kappa$ is stationary. For any $\alpha \in S$, let $\beta \in S$ be such that $\alpha<\beta$. Then $f_{\alpha} \leq^{*} g_{\beta}$ by (3.6). Hence there is $n \in \omega$ such that $f_{\alpha} \leq g_{\beta, n}$. By (3.9) and (3.10), it follows that $a_{\alpha, 0} \cap a_{\beta, n+1}=\emptyset$. This shows that $\langle\mathcal{A}, T\rangle \mid \neq(\mathrm{c} 0)$.

Suppose now that $S_{0}, S_{1} \subseteq \kappa$ are stationary and $\langle 0, n\rangle \in T$. By the definition of $T$, it follows that $n \in \omega \backslash 1$. Let $\alpha \in S_{0}$ and $\beta \in S_{1}$ be such that $\beta<\alpha$. Then, by (3.7), we have $f_{\alpha} \not^{*} g_{\beta}^{+}$. Thus, by (3.9) and (3.10), it follows that $a_{\alpha, 0} \cap a_{\beta, n} \neq \emptyset$. This shows that $\langle\mathcal{A}, T\rangle \mid \neq(\mathrm{c} 1)$.

(d) This follows easily from (c).

(e) Suppose that $\kappa \in \mathfrak{D O}$ and let $\langle X, R\rangle$ be such that $X \subseteq \mathcal{P}(\omega), R$ is a projective binary relation and $\operatorname{otp}\left(\left\langle X, R \cap X^{2}\right\rangle\right)=\kappa$. Let $f: \kappa \rightarrow \mathcal{P}(\omega)$ be the mapping sending $\alpha<\kappa$ to the $\alpha$ th element of $X$ with respect to $R$. Let

$$
A=R \cup \bigcup_{k \in \omega \backslash\{2\}}((\mathcal{P}(\omega)))^{k} .
$$

Then it is easily seen that $\langle f, A\rangle \mid \neq(\mathrm{h} 0)$ and $\langle f, A\rangle \mid \neq(\mathrm{h} 1)$.

(f) This follows from (d) since $\mathfrak{D O}$ is downward closed.

(Lemma 3.7)

Corollary 3.8 .

(a) $\operatorname{HP}(\kappa)$ implies $\min \{\operatorname{non}(I), \operatorname{cov}(I)\} \leq \kappa$ for any ideal $I$ over $\mathbb{R}$ with Borel basis. In particular, it implies

$$
\begin{aligned}
\min \{\text { non }(\text { meager }), \operatorname{cov}(\text { meager })\} & \leq \kappa, \\
\min \{\text { non }(\text { null }), \operatorname{cov}(\text { null })\} & \leq \kappa .
\end{aligned}
$$

(b) If $\mathfrak{d} \mathfrak{o}$ is attained then $\operatorname{HP}(\kappa)$ implies $\min \{\operatorname{non}(I), \operatorname{cov}(I)\}<\kappa$ for all $I$ over $\mathbb{R}$ with Borel basis. In particular, it implies

$$
\begin{aligned}
\min \{\text { non }(\text { meager }), \operatorname{cov}(\text { meager })\} & <\kappa, \\
\min \{\text { non }(\text { null }), \operatorname{cov}(\text { null })\} & <\kappa .
\end{aligned}
$$

Proof. By Lemmas 3.6 and 3.7(f). - (Corollary 3.8)

Corollary 3.9 .

(a) $\mathrm{C}^{\mathrm{S}}\left(\aleph_{2}\right)\left(\right.$ or $\left.\hat{\mathrm{C}}^{\mathrm{S}}\left(\aleph_{2}\right)\right)$ implies $\mathfrak{b}^{h}=\aleph_{1}$.

(b) $\mathrm{HP}\left(\aleph_{2}\right)$ implies

$$
\begin{aligned}
\mathfrak{d} \mathfrak{o} & =\min \{\text { non }(\text { meager }), \operatorname{cov}(\text { meager })\} \\
& =\min \{\text { non }(\text { null }), \operatorname{cov}(\text { null })\}=\aleph_{1} .
\end{aligned}
$$

Proof. (a) By Lemma 3.7(d).

(b) By Lemma 3.7(f) and Corollary 3.8. - (Corollary 3.9) 


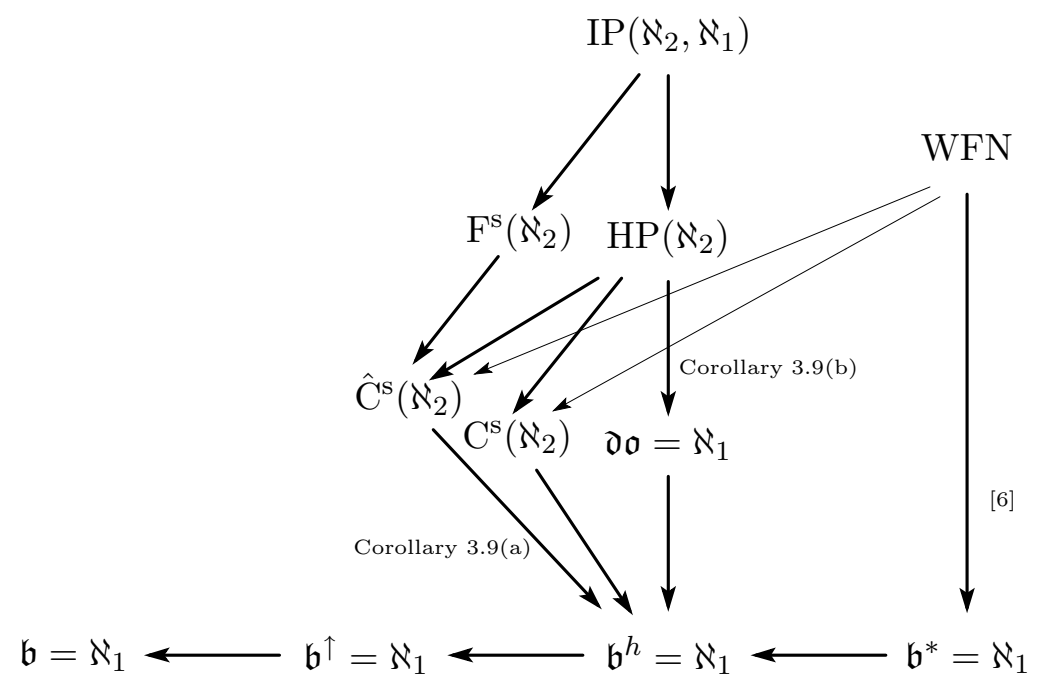

Fig. 5

4. A forcing construction of models of $\operatorname{IP}(\kappa, \lambda)$. In this section, we shall prove that $\operatorname{IP}(\kappa, \lambda)$ holds in a generic extension by a homogeneous product of copies of a relatively small partial ordering (Theorem 4.3).

Let us begin by defining some notions needed for a precise formulation of the theorem.

For cardinals $\kappa$ and $\mu, \kappa$ is said to be $\mu$-inaccessible if $\kappa$ is regular and $\lambda^{\mu}<\kappa$ for all $\lambda<\kappa$. Similarly, we say that $\kappa$ is $<\mu$-inaccessible if $\kappa$ is regular and $\lambda^{<\mu}<\kappa$ for all $\lambda<\kappa$. Thus, if $\mu$ is a successor cardinal, say $\mu=\mu_{0}^{+}$, then $\kappa$ is $<\mu$-inaccessible if and only if $\kappa$ is $\mu_{0}$-inaccessible. In our context, $<\mu$-inaccessibility is relevant because of the following variant of the $\Delta$-system lemma of Erdős and Rado. For cardinals $\mu<\kappa$, let

$$
E_{\geq \mu}^{\kappa}=\{\alpha<\kappa: \operatorname{cf}(\alpha) \geq \mu\}
$$

and let $E_{\mu}^{\kappa}, E_{\leq \mu}^{\kappa}$ etc. be defined analogously.

Theorem 4.1 (P. Erdős and R. Rado, see [13]). Suppose that $\kappa$ is $<\mu$ inaccessible and $S \subseteq E_{\geq \mu}^{\kappa}$ is stationary in $\kappa$. For any sequence $\left\langle x_{\alpha}: \alpha \in S\right\rangle$ of sets of cardinality $\left\langle\mu\right.$ there is a stationary $S^{*} \subseteq S$ such that $\left\langle x_{\alpha}: \alpha \in S^{*}\right\rangle$ form a $\Delta$-system.

For a sequence $\mathbb{P}_{\alpha}, \alpha<\delta$, of posets and an ideal $I \subseteq \mathcal{P}(\delta)$, we consider the I-support product $\prod_{\alpha<\delta}^{I} \mathbb{P}_{\alpha}$ of $\mathbb{P}_{\alpha}, \alpha<\delta$, defined as

$$
\begin{aligned}
& \prod_{\alpha<\delta}^{I} \mathbb{P}_{\alpha}=\left\{f: f: D \rightarrow \bigcup_{\alpha<\delta} \mathbb{P}_{\alpha} \text { for some } D \in I\right. \\
& \text { and } \left.f(\alpha) \in \mathbb{P}_{\alpha} \backslash\left\{\mathbb{1}_{\mathbb{P}_{\alpha}}\right\} \text { for all } \alpha \in D\right\}
\end{aligned}
$$


with the ordering

$$
\begin{aligned}
& f \leq_{\prod_{\alpha<\delta}^{I} \mathbb{P}_{\alpha}} g \Leftrightarrow \operatorname{dom}(f) \supseteq \operatorname{dom}(g) \text { and } \\
& \quad f(\alpha) \leq_{\mathbb{P}_{\alpha}} g(\alpha) \text { for all } \alpha \in \operatorname{dom}(g)
\end{aligned}
$$

for all $f, g \in \prod_{\alpha<\delta}^{I} \mathbb{P}_{\alpha}$. In particular, $\mathbb{1}_{\prod_{\alpha<\delta}^{I} \mathbb{P}_{\alpha}}=\emptyset$ is the largest element of

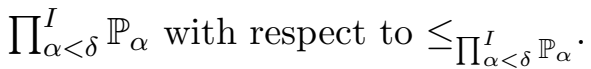

Though this definition of product of posets is different from the standard one, it gives a poset forcing equivalent to the product given by the standard definition. The present definition is chosen here for the sake of smoother treatment of $p\lceil X, P\lceil X, G\lceil X$ etc. (see (4.5), (4.7) etc.)

As usual, the ideal $[\delta]^{<\aleph_{0}}$ is denoted by fin and $\prod_{\alpha<\delta}^{\mathrm{fin}} \mathbb{P}_{\alpha}$ is called the finite support product of $\mathbb{P}_{\alpha}, \alpha<\delta$.

I. Juhász and K. Kunen [12] proved the following theorem for $\mu=\aleph_{1}$ and $I=[\delta]^{<\aleph_{0}}$. Their proof also applies to the following slight generalization.

Theorem 4.2 (I. Juhász and K. Kunen [12]). Suppose that $\mathbb{P}=\prod_{\alpha<\delta}^{I} \mathbb{P}_{\alpha}$ for some ideal $I \subseteq \mathcal{P}(\delta), \mathbb{P}$ satisfies the $\mu$-c.c. and $\left|\mathbb{P}_{\alpha}\right| \leq 2^{<\mu}$ for all $\alpha<\delta$. Then for all $<\mu$-inaccessible $\kappa$ we have $\|_{\mathbb{P}}$ " $\mathrm{C}^{\mathrm{S}}(\kappa)$ ".

Suppose that $I \subseteq \mathbb{P}(\delta)$ is an ideal and $\mathbb{P}=\prod_{\alpha<\delta}^{I} \mathbb{P}_{\alpha}$ is an $I$-support product of posets $\mathbb{P}_{\alpha}, \alpha<\delta$. For $p \in \mathbb{P}$, the support $\operatorname{supp}(p)$ of $p$ is defined by

$$
\operatorname{supp}(p)=\operatorname{dom}(p)
$$

We assume in the following that $\mathbb{P}$-names are constructed just as in [15]. For a $\mathbb{P}$-name $\dot{a}$, the support $\operatorname{supp}(\dot{a})$ is defined by

$$
\operatorname{supp}(\dot{a})=\bigcup\{\operatorname{supp}(p):\langle\dot{b}, p\rangle \in \operatorname{tcl}(\dot{a}) \text { for some } \mathbb{P} \text {-name } \dot{b}\} \text {. }
$$

For $X \in \mathcal{P}(\delta)$ (not necessarily in $I$ ), let

$$
\mathbb{P}\lceil X=\{p\lceil X: p \in \mathbb{P}\} .
$$

By (4.1) and since $I$ is an ideal, we have

$$
\mathbb{P}\lceil X=\{p \in \mathbb{P}: \operatorname{supp}(p) \subseteq X\} .
$$

In particular,

$$
\mathbb{P} \uparrow X \subseteq \mathbb{P}
$$

Furthermore, it is easy to see that $\mathbb{P}\lceil X \Subset \mathbb{P}$. Thus, if $G$ is a $(V, \mathbb{P})$-generic filter then $G \cap(\mathbb{P}\lceil X)$ is a $(V, \mathbb{P}\lceil X)$-generic filter. We shall denote the generic filter $G \cap\left(\mathbb{P}\lceil X)\right.$ by $G_{X}$. Note that a $\mathbb{P}$-name $\dot{a}$ is a $\mathbb{P}\lceil X$-name if and only if $\operatorname{supp}(\dot{a}) \subseteq X$. 
We shall call an $I$-support product $\mathbb{P}=\prod_{\alpha<\delta}^{I} \mathbb{P}_{\alpha}$ homogeneous if $\mathbb{P}_{\alpha} \cong \mathbb{P}_{\beta}$ for all $\alpha, \beta<\delta$ and $I$ is translation invariant, that is, $I=\left\{j^{\prime \prime} x: x \in I\right\}$ for all bijections $j: \delta \rightarrow \delta$.

Note that if $I$ is translation invariant then $I=[\delta]<\lambda$ for some $\lambda$.

For a homogeneous $\mathbb{P}=\prod_{\alpha<\delta}^{I} \mathbb{P}_{\alpha}$, we shall always assume that a commutative system $i_{\alpha, \beta}: \mathbb{P}_{\alpha} \cong \mathbb{P}_{\beta}, \alpha, \beta<\delta$, of isomorphisms is fixed. With such a fixed system of isomorphisms, every bijection $j: \delta \rightarrow \delta$ induces an isomorphism $\tilde{j}: \mathbb{P} \cong \mathbb{P}$ defined by

$$
\begin{aligned}
& \operatorname{dom}(\tilde{j}(p))=j^{\prime \prime} \operatorname{dom}(p) \\
& \text { for } \alpha \in \operatorname{dom}(\tilde{j}(p)), \quad \tilde{j}(p)(\alpha)=i_{j^{-1}(\alpha), \alpha} \circ p \circ j^{-1}(\alpha)
\end{aligned}
$$

for all $p \in \mathbb{P}$.

For notational simplicity we shall denote the isomorphism on $\mathbb{P}$-names induced from $\tilde{j}$ also by $\tilde{j}$.

Note that for $\mathbb{P}$ and $j$ as above, $p \in \mathbb{P}, \mathbb{P}$-names $\dot{a}_{0}, \ldots, \dot{a}_{n-1}$ and a formula $\varphi$ in the language of set theory $\mathcal{L}_{\mathrm{ZF}}$, we have

$$
\begin{aligned}
& p \models_{\mathbb{P}} \text { " } \varphi\left(\dot{a}_{0}, \ldots, \dot{a}_{n-1}\right) " \text { if and only if } \\
& \qquad \tilde{j}(p) \models_{\mathbb{P}} " \varphi\left(\tilde{j}\left(\dot{a}_{0}\right), \ldots, \tilde{j}\left(\dot{a}_{n-1}\right)\right) " .
\end{aligned}
$$

We are now ready to formulate the main result of the present section:

Theorem 4.3. Suppose that

(4.10) $\lambda$ is a regular uncountable cardinal with $2^{<\lambda}=\lambda, \mu \in\left\{\lambda, \lambda^{+}\right\}$and $\kappa$ is a $<\lambda$-inaccessible cardinal.

Let $\mathbb{P}=\prod_{\alpha<\delta}^{I} \mathbb{P}_{\alpha}$ be a homogeneous $I$-support product such that

(4.11) $I \subseteq[\delta]^{<\lambda}$;

(4.12) $\quad\left|\mathbb{P}_{\alpha}\right| \leq \lambda$ for all $\alpha<\delta$ and $\mathbb{P}$ satisfies the $\mu$-c.c.;

(4.13) $\mathbb{P}$ is proper.

Then $\Vdash_{\mathbb{P}}$ "IP $(\kappa, \mu)$ " holds.

The proof of Theorem 4.3 will be given after Lemmas 4.4 to 4.7 belov.

As in [15], a $\mathbb{P}$-name $\dot{x}$ of a subset of $\omega$ for a poset $\mathbb{P}$ is called a nice $\mathbb{P}$-name if there are antichains $A_{\dot{x}, n}, n \in \omega$, in $\mathbb{P}$ such that $\dot{x}=\left\{\langle\check{n}, p\rangle: p \in A_{\dot{x}, n}\right\}$. Note that, for such a name $\dot{x}$, we have $\operatorname{supp}(\dot{x})=\bigcup_{n \in \omega} A_{\dot{x}, n}$. It is easy to see that, for all $\mathbb{P}$-names $\dot{x}$ of subsets of $\omega$, there is a nice $\mathbb{P}$-name $\dot{x}^{\prime}$ such that $\mathbb{P}_{\mathbb{P}}$ " $\dot{x}=\dot{x}^{\prime}$ ". We say that a nice $\mathbb{P}$-name of a subset of $\omega$ with $A_{\dot{x}, n}$, $n \in \omega$, as above is slim if $A_{\dot{x}, n}$ is countable for all $n<\omega$.

The following lemmas are well-known:

Lemma 4.4. Suppose that $\mathbb{P}$ is a proper poset and $p \in \mathbb{P}$. For any $\mathbb{P}_{-}$ name $\dot{x}$ of a subset of $\omega$, there are $q \leq_{\mathbb{P}} p$ and a slim $\mathbb{P}$-name $\dot{x}^{\prime}$ such that $q \Vdash_{\mathbb{P}} " \dot{x}=\dot{x}^{\prime} "$. 
Proof. By the remark above, we may assume without loss of generality that $\dot{x}$ is a nice $\mathbb{P}$-name. Let $A_{\dot{x}, n}, n \in \omega$, be as above and $\dot{y}$ be a $\mathbb{P}$-name such that $\Vdash_{\mathbb{P}}$ " $\dot{y}=\left\{s \in \mathbb{P}: s \in\left(\bigcup_{n \in \omega} A_{\dot{x}, n}\right) \cap \dot{G}\right\}$ ". Then $\models_{\mathbb{P}}$ " $\dot{y}$ is a countable subset of $\mathbb{P} "$. As $\mathbb{P}$ is proper there exist $q \leq \mathbb{P} p$ and countable $y \subseteq \mathbb{P}$ such that $q \Vdash_{\mathbb{P}}$ " $\dot{y} \subseteq y$ ". Let $\dot{x}^{\prime}=\left\{\langle\check{n}, s\rangle: n \in \omega, s \in A_{\dot{x}, n} \cap y\right\}$. These $q$ and $\dot{x}^{\prime}$ are as desired. - (Lemma 4.4)

Lemma 4.5. Suppose that $\mathbb{P}=\prod_{\alpha<\delta}^{I} \mathbb{P}_{\alpha}$ is a $\kappa$-c.c. I-support product for an ideal $I \subseteq[\delta]^{<\lambda}$ and $\kappa$ is $<\lambda$-inaccessible. If $S \subseteq E_{\geq \lambda}^{\kappa}$ is stationary and $p_{\alpha} \in \mathbb{P}$ for $\alpha \in S$ are such that $\operatorname{supp}\left(p_{\alpha}\right), \alpha \in S$, form a $\Delta$-system with the root $R$ and there is $p^{*} \in \mathbb{P}\left\lceil R\right.$ such that $p_{\alpha}\left\lceil R=p^{*}\right.$ for all $\alpha \in S$, then

$$
p^{*} \Vdash_{\mathbb{P}} \text { " }\left\{\alpha \in S: p_{\alpha} \in \dot{G}\right\} \text { is stationary" }
$$

where $\dot{G}$ denotes the standard $\mathbb{P}$-name of a $(V, \mathbb{P})$-generic filter.

Proof. By $\kappa$-c.c. of $\mathbb{P}, \kappa$ remains a regular cardinal in $\mathbb{P}$-generic extensions. Let $\dot{S}$ be a $\mathbb{P}$-name of $\left\{\alpha \in S: p_{\alpha} \in \dot{G}\right\}$. Suppose that $\dot{C}$ is a $\mathbb{P}$-name of a club subset of $\kappa$ and $p \leq \mathbb{P} p^{*}$. It is enough to show that there is a $q \leq \mathbb{P} p$ such that $q \|_{\mathbb{P}} " \dot{C} \cap \dot{S} \neq \emptyset "$.

Let $\theta$ be sufficiently large and let $M \prec \mathcal{H}(\theta)$ be such that

$$
\begin{aligned}
& I, \mathbb{P}, \kappa, \dot{C},\left\langle p_{\alpha}: \alpha \in S\right\rangle, p \in M \\
& |M|<\kappa \cap M<\kappa ; \\
& {[M]^{<\lambda} \subseteq M ;} \\
& \alpha^{*} \in S \text { where } \alpha^{*}=\kappa \cap M .
\end{aligned}
$$

The inclusion (4.16) is possible since $\kappa$ is $<\lambda$-inaccessible. (4.17) is possible since $S \subseteq E_{\geq \lambda}^{\kappa}$ and $S$ is stationary in $\kappa$.

Claim 4.5.1. $\|_{\mathbb{P}}$ " $\alpha^{*} \in \dot{C}$ ".

$\vdash$ Since $\mathbb{P}$ satisfies the $\kappa$-c.c., we have

$$
\mathcal{H}(\theta) \models \forall \alpha<\kappa \exists \beta \in \kappa \backslash \alpha\left(\|_{\mathbb{P}} " \beta \in \dot{C} "\right) .
$$

By (4.14) and elementarity of $M$ it follows that

$$
M \models \forall \alpha<\kappa \exists \beta \in \kappa \backslash \alpha\left(\Vdash_{\mathbb{P}} " \beta \in \dot{C} "\right) .
$$

Thus $\models_{\mathbb{P}}$ " $\dot{C} \cap \alpha^{*}$ is unbounded in $\alpha^{*}$ ". Since $\models_{\mathbb{P}}$ " $\dot{C}$ is a club in $\kappa$ ", it follows that $\models_{\mathbb{P}}$ " $\alpha^{*} \in \dot{C} " . \dashv$ (Claim 4.5.1)

CLAim 4.5.2. $\operatorname{supp}\left(p_{\alpha^{*}}\right) \cap M=R$ and $\operatorname{supp}(p) \cap \operatorname{supp}\left(p_{\alpha^{*}}\right)=R$.

$\vdash$ Suppose $u=\left(\operatorname{supp}\left(p_{\alpha^{*}}\right) \cap M\right) \backslash R \neq \emptyset$. By (4.16), $u \in M$. Hence by elementarity $M \models \exists \alpha<\kappa\left(u \subseteq \operatorname{supp}\left(p_{\alpha}\right)\right)$. Let $\alpha \in \kappa \cap M$ be such that $u \subseteq \operatorname{supp}\left(p_{\alpha}\right)$. Then $\alpha<\alpha^{*}$ and $R \cup u \subseteq p_{\alpha} \cap p_{\alpha^{*}}$. This contradicts the assumption that $R$ is the root of the $\Delta$-system $\left\{\operatorname{supp}\left(p_{\alpha}\right): \alpha \in S\right\}$. Therefore $\operatorname{supp}\left(p_{\alpha^{*}}\right) \cap M=R$. 
By (4.14) and (4.16), $\operatorname{supp}(p) \in M$. It follows that $\operatorname{supp}(p) \cap \sup \left(p_{\alpha^{*}}\right)=$ $\operatorname{supp}(p) \cap\left(\sup \left(p_{\alpha^{*}}\right) \cap M\right)=\operatorname{supp}(p) \cap R=R . \dashv($ Claim 4.5.2)

Since $p\left\lceil R \leq \mathbb{P} p^{*} \mid R=p^{*}=p_{\alpha^{*}} \uparrow R\right.$, we see that $q=p \cup p_{\alpha^{*}} \in \mathbb{P}$. We have $q \leq_{\mathbb{P}} p$. By $p_{\alpha^{*}} \|_{\mathbb{P}}$ " $\alpha^{*} \in \dot{S}$ " and $q \leq \mathbb{P} p_{\alpha^{*}}$, we have $q \nVdash_{\mathbb{P}}$ " $\alpha^{*} \in \dot{C} \cap \dot{S}$ ". In particular, $q \Vdash_{\mathbb{P}}$ " $\dot{C} \cap \dot{S} \neq \emptyset$ ". - (Lemma 4.5)

The arguments for the following two lemmas are also well-known. For Lemma 4.6 see e.g. [12].

Lemma 4.6. Suppose that $\mathbb{P}=\prod_{\alpha<\delta}^{I} \mathbb{P}_{\alpha}$ is an I-support product and $G$ is a $(V, \mathbb{P})$-generic filter. For $X, Y \subseteq \delta$, let $Z=X \cap Y$. Then, in $V[G]$, for any $\kappa \in \operatorname{Card}^{V[G]}$, we have

$$
[\mathrm{On}]^{<\kappa} \cap\left(V\left[G_{X}\right] \backslash V\left[G_{Z}\right]\right) \cap\left(V\left[G_{Y}\right] \backslash V\left[G_{Z}\right]\right)=\emptyset .
$$

Lemma 4.7. Suppose that $\kappa \leq \delta$ and $\mathbb{P}=\prod_{\alpha<\delta}^{I} \mathbb{P}_{\alpha}$ is a $\kappa$-c.c. homogeneous $I$-support product, $p \in \mathbb{P}, \dot{a}_{0}, \ldots, \dot{a}_{n-1}$ are $\mathbb{P}$-names with

$$
\operatorname{supp}\left(\dot{a}_{0}\right), \ldots, \operatorname{supp}\left(\dot{a}_{n-1}\right) \subseteq X
$$

for some $X \subseteq \delta$ and $\varphi=\varphi\left(x_{0}, \ldots, x_{n-1}\right)$ is a formula in $\mathcal{L}_{\mathrm{ZF}}$ (possibly with some parameters from $V$ ).

(a) If

(4.20) $\delta \backslash X \notin I$,

then $p\left\lceil X \Vdash_{\mathbb{P}} " \varphi\left(\dot{a}_{0}, \ldots, \dot{a}_{n-1}\right) "\right.$.

(b) If

$$
\begin{aligned}
& p \nVdash_{\mathbb{P}} "\left(\exists x \in{ }^{\omega} \omega\right) \varphi\left(x, \dot{a}_{1}, \ldots, \dot{a}_{n-1}\right) ", \\
& \operatorname{supp}(p) \subseteq X, \\
& \left|X \backslash\left(\operatorname{supp}(p) \cup \operatorname{supp}\left(\dot{a}_{0}\right) \cup \cdots \cup \operatorname{supp}\left(\dot{a}_{n-1}\right)\right)\right| \geq \kappa,
\end{aligned}
$$

then there is a $\mathbb{P}_{X}$-name $\dot{a}$ such that $p \|_{\mathbb{P}}$ " $\varphi\left(\dot{a}, \dot{a}_{1}, \ldots, \dot{a}_{n-1}\right)$ ".

Proof. (a) Suppose that $p\left\lceil X \| \forall_{\mathbb{P}}\right.$ " $\varphi\left(\dot{a}_{0}, \ldots, \dot{a}_{n-1}\right)$ ". Then there is $q \leq_{\mathbb{P}}$ $p \nmid X$ such that $q \Vdash_{\mathbb{P}} " \neg \varphi\left(\dot{a}_{0}, \ldots, \dot{a}_{n-1}\right)$ ". Let $j: \delta \rightarrow \delta$ be a bijection such that

$$
\begin{aligned}
& j\left\lceil X=\operatorname{id}_{X},\right. \\
& \left(j^{\prime \prime} \operatorname{supp}(q) \backslash X\right) \cap \operatorname{supp}(p)=\emptyset .
\end{aligned}
$$

Note that the last condition is possible by (4.20). By (4.24) and (4.18), we have

$$
\begin{aligned}
& \tilde{j}(q) \mid X=\tilde{j}(q\lceil X)=q\lceil X, \\
& \tilde{j}\left(\dot{a}_{0}\right)=\dot{a}_{0}, \ldots, \tilde{j}\left(\dot{a}_{n-1}\right)=\dot{a}_{n-1} .
\end{aligned}
$$


By (4.27) and by the choice of $q$, we have $\tilde{j}(q) \Vdash_{\mathbb{P}} " \neg \varphi\left(\dot{a}_{0}, \ldots, \dot{a}_{n-1}\right)$ ". On the other hand, by (4.26), $p$ and $\tilde{j}(q)$ are compatible. This contradicts (4.19).

(b) By the maximal principle, there is a nice $\mathbb{P}$-name $\dot{a}^{\prime}$ of a real such that

$$
p \Vdash_{\mathbb{P}} \text { “ } \varphi\left(\dot{a}^{\prime}, \dot{a}_{1}, \ldots, \dot{a}_{n-1}\right) " .
$$

By the $\kappa$-c.c. of $\mathbb{P}$, we have $\left|\operatorname{supp}\left(\dot{a}^{\prime}\right)\right|<\kappa$. By (4.18), (4.22) and (4.23), we can find a bijection $j: \delta \rightarrow \delta$ such that

(4.28) $j$ on $\operatorname{supp}(p) \cup \operatorname{supp}\left(\dot{a}_{1}\right) \cup \cdots \cup \operatorname{supp}\left(\dot{a}_{n-1}\right)$ is the identity mapping, (4.29) $j^{\prime \prime} \operatorname{supp}\left(\dot{a}^{\prime}\right) \subseteq X$.

By $(4.28), \tilde{j}(p)=p$ and $\tilde{j}\left(\dot{a}_{1}\right)=\dot{a}_{1}, \ldots, \tilde{j}\left(\dot{a}_{n-1}\right)=\dot{a}_{n-1}$. Let $\dot{a}=\tilde{j}\left(\dot{a}^{\prime}\right)$. Then $p \models_{\mathbb{P}} " \varphi\left(\dot{a}, \dot{a}_{1}, \ldots, \dot{a}_{n-1}\right) "$ and $\operatorname{supp}(\dot{a}) \subseteq X$ by $(4.29)$. - (Lemma 4.7)

Proof of Theorem 4.3. By Proposition 2.5, we may assume $\models_{\mathbb{P}}$ " $\kappa \leq 2^{\aleph_{0}}$ ". In particular, by (4.11)-(4.13), we may assume that $\delta \geq \kappa$. By the $\mu$-c.c. of $\mathbb{P}, \mu$ and $\kappa$ remain regular cardinals in the generic extension by $\mathbb{P}$.

Let $G$ be a $(V, \mathbb{P})$-generic filter. In $V[G]$, let $f: \kappa \rightarrow \mathcal{P}(\omega)$ and $g$ : $((\mathcal{P}(\omega)))^{<\omega} \rightarrow \mathcal{P}(\omega)$ be definable, say by a formula $\varphi$. We may assume that $\varphi$ has a real $a \in V[G]$ as its unique parameter. Let $\dot{f}, \dot{a}$ and $\dot{g}$ be $\mathbb{P}$-names of $f$, $a$ and $g$ respectively such that $\models_{\mathbb{P}} " \dot{f}: \kappa \rightarrow \mathcal{P}(\omega) ", \models_{\mathbb{P}} " \dot{g}:((\mathcal{P}(\omega))) \stackrel{<\omega}{\rightarrow}$ $\mathcal{P}(\omega)$ " and

$$
\Vdash_{\mathbb{P}} " \forall \bar{x} \in((\mathcal{P}(\omega))){ }^{<\omega} \forall x \in \mathcal{P}(\omega)\left(\dot{g}(\bar{x})=x \leftrightarrow \mathcal{H}\left(\aleph_{1}\right) \models \varphi(\bar{x}, x, \dot{a})\right) " .
$$

Suppose that, for a $p \in G$,

$$
p \Vdash_{\mathbb{P}} \text { "(i0) for } \operatorname{IP}(\kappa, \mu) \text { does not hold for } \dot{f} \text { and } \dot{g} " .
$$

In particular, we have

$$
p \Vdash_{\mathbb{P}} \text { " } \forall \alpha<\kappa(\{\beta \in \kappa: \dot{f}(\beta)=\dot{f}(\alpha)\} \text { is non-stationary) } " .
$$

CLAIM 4.3.1. There is a stationary $S \subseteq E_{\geq \lambda}^{\kappa}$ such that

$$
p \Vdash_{\mathbb{P}} \text { “ } \dot{f}\lceil S \text { is } 1-1 " .
$$

$\vdash$ By the $\kappa$-c.c. of $\mathbb{P}$ and by (4.32), there are club sets $C_{\alpha} \subseteq \kappa$ (in $V$ ) for each $\alpha<\kappa$ such that

$$
p \models_{\mathbb{P}} " C_{\alpha} \cap\{\beta \in \kappa: \dot{f}(\beta)=\dot{f}(\alpha)\}=\emptyset " .
$$

Then $C=\Delta_{\alpha<\kappa} C_{\alpha}$ is club and $S=E_{\geq \lambda}^{\kappa} \cap C$ has the desired property. $\dashv$ (Claim 4.3.1)

We show that $p$ forces (i1) for $\dot{f}$ and $\dot{g}$. Let $p^{\prime} \leq_{\mathbb{P}} p$. It is enough to show that there is $p^{*} \leq_{\mathbb{P}} p^{\prime}$ forcing (i1).

By Lemma 4.4, Theorem 4.1, (4.10), (4.11) and (4.13), there are $p^{\prime \prime} \leq_{\mathbb{P}} p^{\prime}$, a slim $\mathbb{P}$-name $\dot{a}^{\prime}$ of a real, a stationary $S^{*} \subseteq S$, a sequence $\left\langle\dot{x}_{\alpha}^{\prime}: \alpha \in S^{*}\right\rangle$ of slim $\mathbb{P}$-names and a sequence $\left\langle p_{\alpha}: \alpha \in S^{*}\right\rangle$ of conditions in $\mathbb{P}$ such that 
(i) $p^{\prime \prime} \models_{\mathbb{P}}$ " $\dot{a}=\dot{a}^{\prime} "$,

(ii) $p_{\alpha} \leq_{\mathbb{P}} p^{\prime \prime}$

(iii) $p_{\alpha} \|_{\mathbb{P}}$ " $\dot{f}(\alpha)=\dot{x}_{\alpha}^{\prime}$ " for every $\alpha \in S^{*}$;

(4.34) $d_{\alpha}=\operatorname{supp}\left(p_{\alpha}\right) \cup \operatorname{supp}\left(\dot{a}^{\prime}\right) \cup \operatorname{supp}\left(\dot{x}_{\alpha}^{\prime}\right), \alpha \in S^{*}$ are all of the same cardinality and form a $\Delta$-system with root $R$;

(4.35) for each $\alpha, \beta \in S^{*}$ there is a bijection $j_{\alpha, \beta}: \delta \rightarrow \delta$ such that

(i) $j_{\alpha, \beta} \uparrow\left(\delta \backslash\left(d_{\alpha} \triangle d_{\beta}\right)\right)=\mathrm{id}_{\delta \backslash\left(d_{\alpha} \triangle d_{\beta}\right)}$,

(ii) $j_{\alpha, \beta}{ }^{\prime \prime} d_{\alpha}=d_{\beta}, \tilde{j}_{\alpha, \beta}\left(p_{\alpha}\right)=p_{\beta}$,

(iii) $\tilde{j}_{\alpha, \beta}\left(\dot{x}_{\alpha}^{\prime}\right)=\dot{x}_{\beta}^{\prime}$ for every $\alpha, \beta \in S^{*}$.

Note that, by (4.34), we have

$$
\operatorname{supp}\left(\dot{a}^{\prime}\right)=\operatorname{supp}\left(\dot{a}^{\prime}\right) \cap d_{\alpha} \subseteq R \quad \text { for every } \alpha \in S^{*} .
$$

By (4.35), $p_{\alpha} \backslash R$ for $\alpha \in S^{*}$ are all the same. Let $q=p_{\alpha}\lceil R$ for some/any $\alpha \in S^{*}$. Then $q \leq \mathbb{P} p^{\prime \prime}$ by (4.33)(ii). Let $\dot{S}$ be a $\mathbb{P}$-name such that

$$
\|_{\mathbb{P}} " \dot{S}=\left\{\alpha \in S^{*}: p_{\alpha} \in \dot{G}\right\} " .
$$

By Lemma 4.5, $q \Vdash_{\mathbb{P}}$ " $\dot{S}$ is stationary". Hence, by (4.31),

$$
q \Vdash_{\mathbb{P}} " \exists n \in \omega \forall \alpha<\kappa\left(\left|\dot{g}^{\prime \prime}\left(\left(\dot{f}^{\prime \prime}(\dot{S} \backslash \alpha)\right)\right)^{n}\right| \geq \mu\right) " .
$$

Let $q^{\prime} \leq \mathbb{P} q$ and $n^{*} \in \omega$ be such that

$$
q^{\prime} \vdash_{\mathbb{P}} " \forall \alpha<\kappa\left(\left|\dot{g}^{\prime \prime}\left(\left(\dot{f}^{\prime \prime}(\dot{S} \backslash \alpha)\right)\right)^{n^{*}}\right| \geq \mu\right) " .
$$

Let

$$
S^{* *}=\left\{\alpha \in S^{*}: \operatorname{supp}\left(q^{\prime}\right) \cap d_{\alpha} \subseteq R\right\} .
$$

Since $\left|\operatorname{supp}\left(q^{\prime}\right)\right|<\lambda$ by $(4.11)$, it follows that

$$
S^{*} \backslash S^{* *} \text { is of cardinality }<\lambda .
$$

In particular $S^{* *}$ is still stationary and by (4.39),

$$
q^{\prime} \Vdash_{\mathbb{P}} "\left|\dot{g}^{\prime \prime}\left(\left(\dot{f}^{\prime \prime}\left(\dot{S} \cap S^{* *}\right)\right)\right)^{n^{*}}\right| \geq \mu \text { ". }
$$

Claim 4.3.2. There is $\left\langle\alpha_{0}, \ldots, \alpha_{n^{*}-1}\right\rangle \in\left(\left(S^{* *}\right)\right)^{n^{*}}$ such that

$$
q^{\prime} \cup p_{\alpha_{0}} \cup \cdots \cup p_{\alpha_{n^{*}-1}} \forall \mathbb{P} " \dot{g}\left(\left\langle\dot{x}_{\alpha_{0}}^{\prime}, \ldots, \dot{x}_{\alpha_{n^{*}-1}}^{\prime}\right\rangle\right) \in V\left[\dot{G}_{R}\right] ” .
$$

$\vdash$ Otherwise, we would have

$$
q^{\prime} \cup p_{\beta_{0}} \cup \cdots \cup p_{\beta_{n^{*}-1}} \Vdash \mathbb{P} " \dot{g}\left(\left\langle\dot{x}_{\beta_{0}}^{\prime}, \ldots, \dot{x}_{\beta_{n^{*}-1}}^{\prime}\right\rangle\right) \in V\left[\dot{G}_{R}\right] "
$$

for all $\left\langle\beta_{0}, \ldots, \beta_{n^{*}-1}\right\rangle \in\left(\left(S^{* *}\right)\right)^{n^{*}}$.

Fix $\left\langle\alpha_{0}, \ldots, \alpha_{n^{*}-1}\right\rangle \in\left(\left(S^{* *}\right)\right)^{n^{*}}$ and let

$$
\begin{aligned}
\mathcal{D}=\{r \in \mathbb{P}: & r \leq \mathbb{P} q^{\prime} \cup p_{\alpha_{0}} \cup \cdots \cup p_{\alpha_{n^{*}-1}}, \\
& \operatorname{supp}(r) \subseteq R \cup \bigcup\left\{d_{\alpha_{i}}: i<n^{*}\right\} \cup \operatorname{supp}\left(q^{\prime}\right), \\
& r \Vdash_{\mathbb{P}} " \dot{g}\left(\dot{x}_{\alpha_{0}}^{\prime}, \ldots, \dot{x}_{\alpha_{n^{*}-1}}^{\prime}\right)=\dot{x} \text { " for some } \mathbb{P}_{\left.R^{\text {-name }} \dot{x}\right\} .}
\end{aligned}
$$


Let $\mathcal{A}$ be a maximal antichain in $\mathcal{D}$. By the $\mu$-c.c. of $\mathbb{P},|\mathcal{A}|<\mu$. For each $r \in \mathcal{A}$, let $\dot{x}_{r}$ be a $\mathbb{P}_{R}$-name such that

$$
r \Vdash_{\mathbb{P}} " \dot{g}\left(\dot{x}_{\alpha_{0}}^{\prime}, \ldots, \dot{x}_{\alpha_{n^{*}-1}}^{\prime}\right)=\dot{x}_{r} "
$$

and $\dot{\mathcal{X}}$ be a $\mathbb{P}_{R}$-name such that

$$
\|_{\mathbb{P}} " \dot{\mathcal{X}}=\left\{\dot{x}_{r}: r \in \mathcal{A}\right\} " .
$$

Then $\|_{\mathbb{P}}$ " $|\dot{\mathcal{X}}|<\mu$ ". By Lemma $4.7(\mathrm{a})$,

$$
q^{\prime} \cup p_{\alpha_{0}} \cup \cdots \cup p_{\alpha_{n^{*}-1}} \Vdash_{\mathbb{P}} " \dot{g}\left(\left\langle\dot{x}_{\alpha_{0}}^{\prime}, \ldots, \dot{x}_{\alpha_{n^{*}-1}}^{\prime}\right\rangle\right) \in \dot{\mathcal{X}} " .
$$

Hence by (4.35) and (4.9), we have

$$
q^{\prime} \cup p_{\beta_{0}} \cup \cdots \cup p_{\beta_{n^{*}-1}} \Vdash_{\mathbb{P}} " \dot{g}\left(\left\langle\dot{x}_{\beta_{0}}^{\prime}, \ldots, \dot{x}_{\beta_{n^{*}-1}}^{\prime}\right\rangle\right) \in \dot{\mathcal{X}} "
$$

for all $\left\langle\beta_{0}, \ldots, \beta_{n^{*}-1}\right\rangle \in\left(\left(S^{* *}\right)\right)^{n^{*}}$. But this contradicts (4.42). $\dashv$ (Claim 4.3.2)

Let $\left\langle\alpha_{0}, \ldots, \alpha_{n^{*}-1}\right\rangle \in\left(\left(S^{* *}\right)\right)^{n^{*}}$ be as in Claim 4.3.2 and

$$
q^{\prime \prime}=q^{\prime} \cup p_{\alpha_{0}} \cup \cdots \cup p_{\alpha_{n^{*}-1}} .
$$

Note that

$$
q^{\prime \prime} \Vdash_{\mathbb{P}} " f\left(\alpha_{i}\right)=\dot{x}_{\alpha_{i}}^{\prime} " \quad \text { for } i<n^{*}
$$

by (4.43). Let $p^{*} \leq_{\mathbb{P}} q^{\prime \prime}$ be such that

$$
p^{*} \models_{\mathbb{P}} " \dot{g}\left(\dot{x}_{\alpha_{0}}^{\prime}, \ldots, \dot{x}_{\alpha_{n^{*}-1}}^{\prime}\right) \notin V\left[\dot{G}_{R}\right] " .
$$

By thinning out $S^{* *}$ further if necessary, we may assume that $\operatorname{supp}\left(p^{*}\right) \cap$ $\operatorname{supp}\left(p_{\alpha}\right) \subseteq R$ for all $\alpha \in S^{* *}$. For $i<n^{*}$, let $\dot{S}_{i}$ be a $\mathbb{P}$-name such that

$$
\models_{\mathbb{P}} " \dot{S}_{i}=\left\{\alpha \in S^{* *}: \tilde{j}_{\alpha_{i}, \alpha}\left(p^{*}\right) \in \dot{G}\right\} " .
$$

By Lemma 4.5 , we have $p^{*} \|_{\mathbb{P}}$ " $\dot{S}_{i}$ is a stationary subset of $\kappa$ " for all $i<n^{*}$. Note that $\tilde{j}_{\alpha_{i}, \alpha}\left(p^{*}\right) \leq_{\mathbb{P}} p_{\alpha}$ by (4.43) and (4.35)(ii).

Claim 4.3.3. $p^{*} \models_{\mathbb{P}} " \forall \beta_{0} \cdots \forall \beta_{n^{*}-1}\left(\left\langle\beta_{0}, \ldots, \beta_{n^{*}-1}\right\rangle \in\left(\left(\dot{S}_{0}, \ldots, \dot{S}_{n^{*}-1}\right)\right)\right.$

$$
\left.\rightarrow \dot{g}\left(\left\langle\dot{f}\left(\beta_{0}\right), \ldots, \dot{f}\left(\beta_{n^{*}-1}\right)\right\rangle\right) \notin V\left[\dot{G}_{R}\right]\right) "
$$

$\vdash$ Suppose that $q \leq \mathbb{P} p^{*}$ and $q \nVdash_{\mathbb{P}} "\left\langle\beta_{0}, \ldots, \beta_{n^{*}-1}\right\rangle \in\left(\left(\dot{S}_{0}, \ldots, \dot{S}_{n^{*}-1}\right)\right)$ ". Then, by (4.46), $q \Vdash_{\mathbb{P}}$ " $\tilde{j}_{\alpha_{i}, \beta_{i}}\left(p^{*}\right) \in \dot{G}$ " for $i<n^{*}$. It follows that

$$
q \Vdash \mathbb{P} \text { " } \tilde{j}_{\alpha_{i}, \beta_{i}}\left(p^{*}\right)\left\lceil d_{\beta_{i}} \in \dot{G} " \quad \text { for } i<n^{*} .\right.
$$

Let

$$
\tilde{j}=\tilde{j}_{\alpha_{0}, \beta_{0}} \circ \tilde{j}_{\alpha_{1}, \beta_{1}} \circ \cdots \circ \tilde{j}_{\alpha_{n^{*}-1}, \beta_{n^{*}-1}} .
$$

Then

$$
\begin{gathered}
\tilde{j}\left(p^{*}\right)=p^{*} \uparrow\left(\delta \backslash \bigcup_{i<n^{*}} d_{\alpha_{i}}\right) \cup \tilde{j}_{\alpha_{0}, \beta_{0}}\left(p^{*}\right)\left\lceil d_{\beta_{0}}\right. \\
\cup \cdots \cup \tilde{j}_{\alpha_{n^{*}-1}, \beta_{n^{*}-1}}\left(p^{*}\right)\left\lceil d_{\beta_{n^{*}-1}}\right.
\end{gathered}
$$


by (4.35). Hence

$$
q \Vdash_{\mathbb{P}} \text { “ } \tilde{j}\left(p^{*}\right) \in \dot{G} "
$$

by $q \leq_{\mathbb{P}} p^{*}$ and (4.47) and (4.48). By definition of $\tilde{j}$ and $q^{\prime \prime}$, and by (4.35), we have

$$
\begin{gathered}
\tilde{j}\left(p^{*}\right) \leq_{\mathbb{P}} \tilde{j}\left(q^{\prime \prime}\right) \leq_{\mathbb{P}} \tilde{j}_{\alpha_{i}, \beta_{i}}\left(p_{\alpha_{i}}\right)=p_{\beta_{i}} \quad \text { for } i<n^{*}, \\
\tilde{j}\left(\dot{x}_{\alpha_{i}}^{\prime}\right)=\dot{x}_{\beta_{i}}^{\prime} \quad \text { for } i<n^{*}
\end{gathered}
$$

by (4.44). Hence by (4.45),

$$
q \Vdash \mathbb{P} " \dot{g}\left(\left\langle\dot{x}_{\beta_{0}}^{\prime}, \ldots, \dot{x}_{\beta_{n^{*}-1}}^{\prime}\right\rangle\right) \notin V\left[\dot{G}_{R}\right] ” .
$$

By (4.33), (4.49) and (4.50), it follows that

$$
q \Vdash_{\mathbb{P}} " \dot{f}\left(\beta_{i}\right)=\dot{x}_{\beta_{i}}^{\prime} " \quad \text { for } i<n^{*} .
$$

Hence $q \Vdash_{\mathbb{P}}$ “ $\dot{g}\left(\left\langle\dot{f}\left(\beta_{0}\right), \ldots, \dot{f}\left(\beta_{n^{*}-1}\right)\right\rangle\right) \notin V\left[G_{R}\right] " . \dashv($ Claim 4.3.3)

To show that $p^{*} \models_{\mathbb{P}}$ "(i1) holds", suppose that $q \leq \mathbb{P} p^{*}$ and $\left\langle\beta_{0}, \ldots\right.$ $\left.\ldots, \beta_{n^{*}-1}\right\rangle,\left\langle\gamma_{0}, \ldots, \gamma_{n^{*}-1}\right\rangle \in\left(\left(S^{* *}\right)\right)^{n^{*}}$ are such that

$$
\begin{gathered}
\left\{\beta_{0}, \ldots, \beta_{n^{*}-1}\right\} \cap\left\{\gamma_{0}, \ldots, \gamma_{n^{*}-1}\right\}=\emptyset \\
q \mapsto_{\mathbb{P}} "\left\langle\beta_{0}, \ldots, \beta_{n^{*}-1}\right\rangle,\left\langle\gamma_{0}, \ldots, \gamma_{n^{*}-1}\right\rangle \in\left(\left(\dot{S}_{0}, \ldots, \dot{S}_{n^{*}-1}\right)\right) " .
\end{gathered}
$$

Note that it is enough to consider $\left\langle\beta_{0}, \ldots, \beta_{n^{*}-1}\right\rangle,\left\langle\gamma_{0}, \ldots, \gamma_{n^{*}-1}\right\rangle \in\left(\left(S^{* *}\right)\right)^{n^{*}}$ with (4.52) since we can thin out $\dot{S}_{0}^{G}, \ldots, \dot{S}_{n^{*}-1}^{G}$ afterwards if necessary so that they are pairwise disjoint.

By the remark after (4.46), we may assume that

$$
q \leq \mathbb{P} p^{*} \cup p_{\beta_{0}} \cup \cdots \cup p_{\beta_{n^{*}-1}} \cup p_{\gamma_{0}} \cup \cdots \cup p_{\gamma_{n^{*}-1}} .
$$

By Lemma 4.7(b), there are $\mathbb{P}$-names $\dot{y}, \dot{z}$ such that $\operatorname{supp}(\dot{y}) \cap \operatorname{supp}(\dot{z}) \subseteq R$ and

$$
\begin{aligned}
& p^{*} \cup p_{\beta_{0}} \cup \cdots \cup p_{\beta_{n^{*}-1}} \cup p_{\gamma_{0}} \cup \cdots \cup p_{\gamma_{n^{*}-1}} \\
& \quad \mapsto_{\mathbb{P}} " \dot{g}\left(\left\langle\dot{f}\left(\beta_{0}\right), \ldots, \dot{f}\left(\beta_{n^{*}-1}\right)\right\rangle\right)=\dot{y} \wedge \dot{g}\left(\left\langle\dot{f}\left(\gamma_{0}\right), \ldots, \dot{f}\left(\gamma_{n^{*}-1}\right)\right\rangle\right)=\dot{z} " .
\end{aligned}
$$

By Claim 4.3.3 and Lemma 4.6, it follows that

$$
\begin{aligned}
q \leq \mathbb{P} p^{*} \cup p_{\beta_{0}} \cup \cdots \cup p_{\beta_{n^{*}-1}} \cup p_{\gamma_{0}} \cup \cdots \cup p_{\gamma_{n^{*}-1}} \\
\models_{\mathbb{P}} “ \dot{g}\left(\left\langle\dot{f}\left(\beta_{0}\right\rangle, \ldots, \dot{f}\left(\beta_{n^{*}-1}\right)\right) \neq \dot{g}\left(\left\langle\dot{f}\left(\gamma_{0}\right), \ldots, \dot{f} \gamma_{n^{*}-1}\right)\right)\right\rangle " .
\end{aligned}
$$

Since $q$ as above may be chosen below arbitrary $r \leq_{\mathbb{P}} p^{*}$, it follows that $p^{*} \vdash_{\mathbb{P}}$ "(i1) holds".

Corollary 4.8.

(a) Assume $\mathrm{CH}$ and $\mathbb{P}=\operatorname{Fn}(\mu, 2)$ for some cardinal $\mu$. Then $\|_{\mathbb{P}} " \operatorname{IP}\left(\aleph_{2}, \aleph_{1}\right) "$ holds. 
(b) Assume $\mathrm{GCH}$ and $\mathbb{P}=\mathrm{Fn}(\mu, 2)$ for some cardinal $\mu$. Then $\|_{\mathbb{P}}$ "IP $\left(\kappa^{+}, \aleph_{1}\right)$ " for every $\kappa$ of uncountable cofinality and $\|_{\mathbb{P}}$ "IP $\left(\lambda, \aleph_{1}\right)$ " for every inaccessible $\lambda$.

(c) Assume $\mathrm{CH}$ and $\mathbb{P}$ is a finite support product of copies of a productively c.c.c. poset of cardinality $\aleph_{1}$. Then $\models_{\mathbb{P}}$ "IP $\left(\aleph_{2}, \aleph_{1}\right)$ ". In particular, $\|_{\mathbb{P}}$ $\mathrm{HP}\left(\aleph_{2}\right) "$.

(d) Assume GCH and $\mathbb{P}$ is a finite support product of copies of a productively c.c.c. poset of cardinality $\aleph_{1}$. Then $\models_{\mathbb{P}} " \operatorname{IP}\left(\kappa^{+}, \aleph_{1}\right)$ " for every $\kappa$ of uncountable cofinality and $\models_{\mathbb{P}} " \operatorname{IP}\left(\lambda, \aleph_{1}\right)$ " for every inaccessible $\lambda$.

(e) Assume $\mathrm{CH}$ and $\mathbb{P}$ is a countable support product of copies of a proper poset of cardinality $\aleph_{1}$ such that its product is also proper. Then $\Vdash_{\mathbb{P}}$ "IP $\left(\aleph_{2}, \aleph_{2}\right)$ ". In particular, $\Vdash_{\mathbb{P}}$ " $\mathrm{HP}\left(\aleph_{2}\right)$ ".

(f) Assume GCH and $\mathbb{P}$ is a countable support product of copies of a proper poset of cardinality $\aleph_{1}$ such that its product is also proper. Then $\models_{\mathbb{P}}$ "IP $\left(\kappa^{+}, \aleph_{2}\right)$ " for every $\kappa$ of uncountable cofinality and $\|_{\mathbb{P}}$ "IP $\left(\lambda, \aleph_{2}\right)$ " for every inaccessible $\lambda$.

Note that countable support products of Sacks or Prikry-Silver forcing are instances of (e) and (f) above.

Proof. Under CH, $\omega_{1}=2^{<\omega_{1}}$ and $\omega_{2}$ is $<\omega_{1}$-inaccessible. In (a) and (b), $\mathbb{P}$ is forcing equivalent to a finite support product of copies of the countable poset $\mathrm{Fn}(\omega, 2)$. Clearly $\mathbb{P}$ 's in all of (a)-(f) are homogeneous; $\mathbb{P}$ 's in (a)-(d) satisfy the c.c.c. and hence they are proper. Thus we can apply Theorem 4.3. The second parts of (c) and (e) follow from Theorem 2.9. - (Corollary 4.8)

Results similar to Theorem 4.3 and Corollary 4.8 also hold for partial orderings with product-like structure as those considered in [9]. Thus, we can prove e.g. that $\operatorname{IP}\left(\aleph_{2}, \aleph_{2}\right)$ together with clubsuit principle is consistent.

In [5] it is shown that, if we start from a model $V$ which is obtained by adding a dominating real to a model of GCH + Chang's conjecture for $\aleph_{\omega}$, i.e. $\left(\aleph_{\omega+1}, \aleph_{\omega}\right) \rightarrow\left(\aleph_{1}, \aleph_{0}\right)$, then adding more than $\aleph_{\omega+1}$ Cohen reals forces $\neg \mathrm{WFN}$. Since $V$ satisfies $\mathrm{GCH}, \operatorname{IP}\left(\kappa, \aleph_{1}\right)$ is forced for every $\kappa \geq \aleph_{2}$ which is not a successor of a singular cardinal of cofinality $\omega$ by adding any number of Cohen reals by Corollary 4.8. In particular:

COROLlary 4.9. Suppose that Chang's conjecture for $\aleph_{\omega}$ is consistent. Then so is $\operatorname{IP}\left(\aleph_{2}, \aleph_{1}\right) \wedge \mathfrak{b}^{*}=\aleph_{1} \wedge \neg \mathrm{WFN}$.

5. Models of $\operatorname{IP}\left(\aleph_{2}, \aleph_{2}\right) \wedge \neg \operatorname{IP}\left(\aleph_{2}, \aleph_{1}\right)$. Recall that Prikry-Silver forcing $\mathbb{S}$ is the forcing with partial functions with co-infinite domain, that is,

$$
\mathbb{S}=\left\{f: f: D \rightarrow 2, D \subseteq \omega,|\omega \backslash D|=\aleph_{0}\right\}
$$


with the ordering

$$
f \leq \mathbb{S} g \Leftrightarrow f \supseteq g
$$

for $f, g \in \mathbb{S}$.

A $(V, \mathbb{S})$-generic filter $G$ gives rise to the function $s_{G}=\bigcup G: \omega \rightarrow 2$ which is often called a Prikry-Silver real.

For $f \in \mathbb{S}$ let $\operatorname{codom}(f)=\omega \backslash \operatorname{dom}(f)$.

It is easy to check that Prikry-Silver forcing $\mathbb{S}$ as well as its countable support products $\mathbb{S}^{I}$ over any index set $I$ satisfy Axiom A. Hence they are all proper.

Note that, by definition of $\leq_{\mathbb{S}}$, we have:

(5.1) $\quad f, g \in \mathbb{S}$ are incompatible if $|\operatorname{codom}(f) \cap \operatorname{codom}(g)|<\aleph_{0}$.

(5.2) For any $\left\langle f_{0}, f_{1}\right\rangle \in \mathbb{S}^{2}$, there is $\left\langle g_{0}, g_{1}\right\rangle \leq_{\mathbb{S}^{2}}\left\langle f_{0}, f_{1}\right\rangle$ such that $\mid \operatorname{codom}\left(g_{0}\right)$ $\cap \operatorname{codom}\left(g_{1}\right) \mid<\aleph_{0}$.

Lemma 5.1. For any $f \in \mathbb{S}$ and $\left\langle g_{0}^{n}, g_{1}^{n}\right\rangle \in \mathbb{S}^{2}, n \in \omega$, such that $\mid \operatorname{codom}\left(g_{0}^{n}\right)$ $\cap \operatorname{codom}\left(g_{1}^{n}\right) \mid<\aleph_{0}$ there is $g \leq_{\mathbb{S}} f$ such that $\langle g, g\rangle$ is incompatible with all $\left\langle g_{0}^{n}, g_{1}^{n}\right\rangle, n \in \omega$.

Proof. Construct $i_{n} \in 2, n \in \omega$ and $A \subseteq \operatorname{codom}(f)$ recursively so that

$$
\begin{gathered}
\left|\operatorname{codom}(f) \cap \bigcap_{k \leq n} \operatorname{dom}\left(g_{i_{k}}^{k}\right)\right|=\aleph_{0}, \\
\left|A \cap \operatorname{codom}\left(g_{i_{n}}^{n}\right)\right|<\aleph_{0} \quad \text { for all } n \in \omega .
\end{gathered}
$$

Then any extension $g$ of $f$ on $\omega \backslash A$ will do. - (Lemma 5.1)

Working in $V=L$, we can construct recursively a maximal antichain $\left\{\left\langle g_{0}^{\alpha}, g_{1}^{\alpha}\right\rangle: \alpha<\omega_{1}\right\}$ in $\mathbb{S}^{2}$ such that

$$
\left|\operatorname{codom}\left(g_{0}^{\alpha}\right) \cap \operatorname{codom}\left(g_{1}^{\alpha}\right)\right|<\aleph_{0} \text { for all } \alpha<\omega_{1} .
$$

Note that each step of the recursive construction is possible by (5.2) and (5.2). Furthermore by choosing $\left\langle g_{0}^{\alpha}, g_{1}^{\alpha}\right\rangle$ in each step of the construction according to the $\Sigma_{2}^{1}$-well ordering of the reals (which exists because $V=L$ ), we can make $\left\{\left\langle g_{0}^{\alpha}, g_{1}^{\alpha}\right\rangle: \alpha<\omega_{1}\right\}$ a $\Sigma_{2}^{1}$-set (actually we can even choose such a maximal antichain as a $\Pi_{1}^{1}$-set arguing similarly to [16]).

Let $\varphi: \mathbb{S}^{2} \rightarrow{ }^{\omega_{2}}$ be a Borel bijection and define $g:\left(\left({ }^{\omega} 2\right)\right)^{<\omega} \rightarrow{ }^{\omega_{2}}$ by

$$
g\left(\left\langle x_{0}, \ldots, x_{n-1}\right\rangle\right)= \begin{cases}\varphi\left(g_{0}^{\alpha^{*}}, g_{1}^{\alpha^{*}}\right) & \text { if } n=2, \text { there is } \alpha<\omega_{2} \text { with } \\ & x_{0} \supseteq g_{0}^{\alpha}, x_{1} \supseteq g_{1}^{\alpha} \text { and } \alpha^{*} \text { is } \\ & \text { minimal among such } \alpha \text { 's } \\ & \text { otherwise. }\end{cases}
$$

It is easy to check that $g$ is a $\Delta_{3}^{1}$-set. 
TheOREM 5.2. Assume $V=L$. Then

$$
\Vdash_{\mathbb{S}_{2} \omega_{2}} \text { IP }\left(\aleph_{2}, \aleph_{2}\right) \text { and } \neg \operatorname{IP}\left(\aleph_{2}, \aleph_{1}\right) " .
$$

Proof. $\|_{\mathbb{S}^{\omega_{2}}}$ "IP $\left(\aleph_{2}, \aleph_{2}\right)$ " follows from Corollary 4.8(e).

To show that $\|_{\mathbb{S}_{2}}$ " $\neg \operatorname{IP}\left(\aleph_{2}, \aleph_{1}\right)$ ", let $G$ be a $\left(V, \mathbb{S}^{\omega_{2}}\right)$-generic filter. Working in $L[G]$, let $s_{\beta}$ be the $\beta$ th Prikry-Silver real added by $G$ for $\beta<\omega_{2}$. Let $f: \omega_{2} \rightarrow{ }^{\omega} 2$ be defined by

$$
f(\beta)=s_{\beta} \quad \text { for } \beta<\omega_{2}
$$

and let $g:\left(\left(\omega_{2}\right)\right)^{<\omega} \rightarrow{ }^{\omega_{2}}$ be the mapping as in (5.4), or more precisely, let $g$ be the mapping (in $L[G]$ ) defined by the $\Delta_{3}^{1}$ definition corresponding to (5.4).

We show that $f$ and $g$ build a counter-example to $\operatorname{IP}\left(\aleph_{2}, \aleph_{1}\right)$.

Since $|\operatorname{rng}(g)| \leq \aleph_{1}$, (i1) clearly fails for these $f$ and $g$. Hence we will be done by showing that $f$ and $g$ do not satisfy (i0).

Assume otherwise. Returning to $L$, let $\dot{f}, \dot{g}, \dot{s}_{\beta}, \beta<\omega_{2}$ etc. be $\mathbb{S}^{\omega_{2}}$-names of $f, g, s_{\beta}, \beta<\omega_{2}$ etc. respectively. In particular, we can choose $\dot{f}$ such that

$$
\Vdash_{\mathbb{S}^{\omega_{2}}} " \dot{f}(\beta)=\dot{s}_{\beta} " \quad \text { for all } \beta<\omega_{2} .
$$

Since $\mathbb{S}^{\omega_{2}}$ is proper, there are $p \in G, \mathbb{S}^{\omega_{2}}$-name $\dot{S}$ and a countable set $Z$ (in $L$ ) such that

$$
p \Vdash_{\mathbb{S}_{2} \omega_{2}} \text { S } \subseteq \omega_{2} \text { is stationary and } \dot{g}^{\prime \prime}\left(\left(\dot{f}^{\prime \prime} \dot{S}\right)\right)^{2} \subseteq Z " .
$$

Let $U=\left\{\beta<\omega_{2}\right.$ : there is $p^{\prime} \leq_{\mathbb{S}^{\omega_{2}}} p$ such that $\left.p^{\prime} \|_{\mathbb{S}^{\omega_{2}}} " \beta \in \dot{S} "\right\}$. Then $U$ is a stationary subset of $\omega_{2}$. For each $\beta \in U$, let $p_{\beta} \leq_{\mathbb{S}^{\omega_{2}}} p$ be such that $p_{\beta} \Vdash_{\mathbb{S}^{\omega_{2}}} " \beta \in \dot{S}$ " and $\beta \in \operatorname{supp}\left(p_{\beta}\right)$.

By the $\Delta$-system lemma and $\mathrm{CH}$, there is $U^{*} \in[U]^{\aleph_{2}}$ such that

(5.8) $\operatorname{supp}\left(p_{\beta}\right), \beta \in U^{*}$, form a $\Delta$-system with root $R$ which is an initial segment of all of $\operatorname{supp}\left(p_{\beta}\right), \beta \in U^{*}$;

(5.9) $\quad \sup R<\min U^{*}$;

(5.10) $p_{\beta} \backslash R, \beta \in U^{*}$, are all the same;

(5.11) $\quad p_{\beta}(\beta), \beta \in U^{*}$, are all the same, say $h \in \mathbb{S}$.

Note that $p_{\beta}, \beta \in U^{*}$, are compatible by (5.8) and (5.10).

Let

$$
X=\varphi^{-1}(Z) .
$$

By Lemma 5.1 , there is a $k \leq_{\mathbb{S}} h$ such that $\langle k, k\rangle$ is incompatible with all $\left\langle g_{0}^{\alpha}, g_{1}^{\alpha}\right\rangle$ from the countable set $X$. 
Fix two distinct $\beta, \gamma \in U^{*}$ and let $q \leq_{\mathbb{S}^{\omega_{2}}} p_{\beta}, p_{\gamma}$ be defined by $\operatorname{dom}(q)=$ $\operatorname{dom}\left(p_{\beta}\right) \cup \operatorname{dom}\left(p_{\gamma}\right)$ and

$$
q(\delta)= \begin{cases}p_{\beta}(\delta) & \text { if } \delta \in \operatorname{supp}\left(p_{\beta}\right) \backslash\{\beta\}, \\ p_{\gamma}(\delta) & \text { else if } \delta \in \operatorname{supp}\left(p_{\gamma}\right) \backslash\{\gamma\}, \\ k & \text { else if } \delta=\beta \text { or } \delta=\gamma,\end{cases}
$$

for $\delta \in \operatorname{dom}(q)$. Since $q \leq_{\mathbb{S}^{\omega_{2}}} p_{\beta}, p_{\gamma}$, we have $q \Vdash_{\mathbb{S}^{\omega_{2}}}$ " $\beta, \gamma \in \dot{S}$ ". Thus the following claim yields a contradiction to (5.7):

Claim 5.2.1. $q \nVdash_{\mathbb{S}_{2} \omega_{2}} " \dot{g}(\langle\dot{f}(\beta), \dot{f}(\gamma)\rangle) \notin Z$ ".

$\vdash$ By (5.6), we have to show $q \nVdash_{\mathbb{S}^{\omega_{2}}}$ " $\dot{g}\left(\left\langle\dot{s}_{\beta}, \dot{s}_{\gamma}\right\rangle\right) \notin Z$ ".

First, we show that $q \Vdash_{\mathbb{S}^{\omega_{2}}}$ " $\dot{g}\left(\left\langle\dot{s}_{\beta}, \dot{s}_{\gamma}\right\rangle\right) \neq 0$ ". Note that, by the complete embedding $\mathbb{S}^{2} \ni\left\langle g_{0}, g_{1}\right\rangle \mapsto\left\{\left\langle\beta, g_{0}\right\rangle,\left\langle\gamma, g_{1}\right\rangle\right\} \in \mathbb{S}^{\{\beta, \gamma\}} \subseteq \mathbb{S}^{\omega_{2}}$,

$$
\left\{\left\{\left\langle\beta, g_{0}^{\alpha}\right\rangle,\left\langle\gamma, g_{1}^{\alpha}\right\rangle\right\}: \alpha<\omega_{1}\right\} \text { is a maximal antichain in } \mathbb{S}^{\omega_{2}} .
$$

For any $r \leq_{\mathbb{S}^{\omega_{2}}} q$, let $\alpha^{*}<\omega_{1}$ be such that $r$ and $\left\{\left\langle\beta, g_{0}^{\alpha^{*}}\right\rangle,\left\langle\gamma, g_{1}^{\alpha^{*}}\right\rangle\right\}$ are compatible. Let $s \leq_{\mathbb{S}^{\omega_{2}}} r,\left\{\left\langle\beta, g_{0}^{\alpha^{*}}\right\rangle,\left\langle\gamma, g_{1}^{\alpha^{*}}\right\rangle\right\}$. Then

$$
s \Vdash_{\mathbb{S}^{\omega_{2}}} \text { " } \dot{s}_{\beta} \supseteq g_{0}^{\alpha^{*}} \text { and } \dot{s}_{\gamma} \supseteq g_{1}^{\alpha^{*}} \text { ". }
$$

Hence, by (5.4), it follows that $s \Vdash_{\mathbb{S}_{2} \omega_{2}} " \dot{g}\left(\left\langle\dot{s}_{\beta}, \dot{s}_{\gamma}\right\rangle\right) \neq 0$ ".

Now, suppose, for contradiction, that there is $r \leq_{\mathbb{Q}^{w_{2}}} q$ such that

$$
r \Vdash_{\mathbb{S}^{\omega}} \text { " } \dot{g}\left(\left\langle\dot{s}_{\beta}, \dot{s}_{\gamma}\right\rangle\right) \in Z " .
$$

Then, by the first part of the proof, there are $s \leq_{\mathbb{S}_{1} \omega_{1}} r$ and $\left\langle g_{0}^{\alpha}, g_{1}^{\alpha}\right\rangle \in X$ such that $s \|_{\mathbb{S}_{2} \omega_{2}}$ " $\dot{s}_{\beta} \supseteq g_{0}^{\alpha}$ and $\dot{s}_{\gamma} \supseteq g_{1}^{\alpha}$ ". In particular $s(\beta)$ and $s(\gamma)$ are compatible with $g_{0}^{\alpha}$ and $g_{1}^{\alpha}$, respectively. Since $r \leq_{\mathbb{S}_{2} \omega_{2}} q \leq_{\mathbb{S}^{\omega_{2}}}\{\langle\beta, k\rangle,\langle\gamma, k\rangle\}$, it follows that $k$ is compatible with both of $g_{0}^{\alpha}$ and $g_{1}^{\alpha}$. This contradicts the choice of $k$. $\dashv$ (Claim 5.2.1) • (Theorem 5.2)

We can prove a lemma similar to Lemma 5.1 for the $\omega$ product of Sacks forcing. Thus, by a similar argument to the one above, we can also prove that $\operatorname{IP}\left(\aleph_{2}, \aleph_{1}\right)$ fails in a generic extension by countable support side-by-side product of Sacks forcing.

6. The consistency of $\mathfrak{b}^{*}=\aleph_{2} \wedge \mathfrak{d o}=\aleph_{1}$. In the following, (A) will stand for the assertion that there is a structure $\left\langle\left(\omega_{2}\right)^{2}, A, \mathcal{F}\right\rangle$ with the properties (6.1)-(6.5) below. Recall that a mapping $f: X \rightarrow X$ is called an involution if it is a bijection exchanging (some) pairs of elements of $X$, that is, $f \circ f=\mathrm{id}_{X}$.

(6.1) $\omega_{2} \times \omega_{2} \supseteq A \supseteq\left\{\langle\alpha, \beta\rangle \in \omega_{2} \times \omega_{2}: \beta<\alpha\right\}$;

(6.2) For any $C \in\left[\omega_{2}\right]^{\aleph_{0}}$ there is an $X \in\left[\omega_{2}\right]^{\aleph_{2}}$ such that $(C \times X) \cap A=\emptyset$;

(6.3) For all $\langle\phi, \psi\rangle \in \mathcal{F}, \phi$ and $\psi$ are involutions on $\omega_{2}$; 
(6.4) For each $\langle\phi, \psi\rangle \in \mathcal{F}$ and for all $\langle\alpha, \beta\rangle \in \omega_{2} \times \omega_{2}$, we have $\langle\alpha, \beta\rangle \in A$ if and only if $\langle\phi(\alpha), \psi(\beta)\rangle \in A$;

(6.5) For any stationary $S \subseteq E_{\omega_{1}}^{\omega_{2}}$ and any $A_{\zeta}, B_{\zeta} \in\left[\omega_{2}\right]^{\aleph_{0}}$ for $\zeta \in S$, there is a stationary $T \subseteq S$ such that, for any $n \in \omega$, if $\zeta_{i}, \eta_{i} \in T$ for $i \in n$ are pairwise distinct (2n elements) then there is $\langle\phi, \psi\rangle \in \mathcal{F}$ such that $\phi^{\prime \prime} A_{\zeta_{i}}=A_{\eta_{i}}, \psi^{\prime \prime} B_{\zeta_{i}}=B_{\eta_{i}}$, and $\phi\left\lceil A_{\zeta_{i}}: A_{\zeta_{i}} \rightarrow A_{\eta_{i}}\right.$ and $\psi\left\lceil B_{\zeta_{i}}: B_{\zeta_{i}} \rightarrow B_{\eta_{i}}\right.$ are order isomorphisms for all $i \in n$.

The consistency of (A) together with $\mathrm{CH}$ over ZFC is proved in the next section. Below, we will prove the consistency of $\mathfrak{c}=\mathfrak{b}^{*}=\aleph_{2} \wedge \mathfrak{d o}=\aleph_{1} \wedge$ $\neg \mathrm{C}^{\mathrm{S}}\left(\aleph_{2}\right)$ by constructing a model of this combination of assertions starting from a model of (A) and $\mathrm{CH}$.

Let us begin by introducing some notation for the forcing construction we use in the proof.

For a cardinal $\kappa$, a sequence $\bar{f}=\left\langle f_{\xi}: \xi<\kappa\right\rangle$ in ${ }^{\omega} \omega$, and $X \subseteq \kappa$, let $\mathbb{D}_{\bar{f}, X}$ be the canonical poset adding an element of $\omega_{\omega}$ dominating $\left\{f_{\xi}: \xi \in X\right\}$. That is,

$$
\mathbb{D}_{\bar{f}, X}=\left\{\langle s, F\rangle: s \in{ }^{\omega\rangle} \omega, F \in[\kappa]^{<\aleph_{0}}\right\}
$$

and, for $\langle s, F\rangle,\left\langle s^{\prime}, F^{\prime}\right\rangle \in \mathbb{D}_{\bar{f}, X}$,

$$
\begin{aligned}
\left\langle s^{\prime}, F^{\prime}\right\rangle \leq \mathbb{D}_{\bar{f}, X}\langle s, F\rangle \Leftrightarrow & s^{\prime} \supseteq s, F^{\prime} \supseteq F, \\
& \forall \alpha \in F \cap X \forall n \in \operatorname{dom}\left(s^{\prime}\right) \backslash \operatorname{dom}(s) \\
& \quad\left(f_{\alpha}(n) \leq s^{\prime}(n)\right) .
\end{aligned}
$$

Since any $\langle s, F\rangle,\left\langle s^{\prime}, F^{\prime}\right\rangle \in \mathbb{D}_{\bar{f}, X}$ with $s=s^{\prime}$ are compatible, we have:

\section{Lemma 6.1. $\mathbb{D}_{\bar{f}, X}$ is $\sigma$-centered.}

Note that the underlying set of $\mathbb{D}_{\bar{f}, X}$ does not depend on the sequence $\bar{f}$. So we shall denote this set with $\mathbb{D}_{X}$. Actually $\mathbb{D}_{X}$ as a set does not depend on $X$ either. Nevertheless we shall add the suffix $X$ so that we can distinguish $\mathbb{D}$ 's by their intended function.

Note also that, as a set, $\prod_{\alpha<\kappa}^{\mathrm{fin}} \mathbb{D}_{\bar{f}, X_{\alpha}}$ is the same for any $\kappa$-sequence $\bar{f}$ of reals; we shall denote this set by $\prod_{\alpha<\kappa}^{\text {fin }} \mathbb{D}_{X_{\alpha}}$.

If $d \in \mathbb{D}_{X}$ and $d=\langle s, F\rangle$ then we shall write $s^{d}$ and $F^{d}$ to denote these $s$ and $F$ respectively.

In the following we assume that a sequence $\bar{X}=\left\langle X_{\alpha}: \alpha\langle\kappa\rangle\right.$ of nonempty subsets of $\kappa$ is fixed. Let

$$
\mathbb{Q}_{\bar{X}}=\mathbb{C}_{\kappa} * \prod_{\alpha<\kappa}^{\text {fin }} \mathbb{D}_{\dot{\bar{f}}, X_{\alpha}}
$$

where $\mathbb{C}_{\kappa}=\operatorname{Fn}(\kappa \times \omega, \omega)$ and $\dot{\bar{f}}$ denotes the $\mathbb{C}_{\kappa}$-name of the sequence of Cohen reals $\left(\in{ }^{\omega} \omega\right)$ of length $\kappa$ added by $\mathbb{C}_{\kappa}$. Thus, if $G$ is a $\left(V, \mathbb{C}_{\kappa}\right)$-generic 
set and $c_{\alpha}$ is the $\alpha$ th element of $\dot{\bar{f}} G$, then $c_{\alpha}(n)=m$ if and only if there is a condition $c \in G$ such that $\langle\alpha, n\rangle \in \operatorname{dom}(c)$ and $c(\alpha, n)=m$.

Let

$$
\begin{aligned}
& \mathbb{Q}_{\bar{X}}^{\dagger}=\{\langle c, d\rangle: c \in \mathbb{C}_{\kappa}, d \in \prod_{\alpha \in \kappa}^{\mathrm{fin}} \mathbb{D}_{X_{\alpha}}, \\
&\left.\bigcup_{\xi \in \operatorname{dom}(d)} F^{d(\xi)} \times \operatorname{dom}\left(s^{d(\xi)}\right) \subseteq \operatorname{dom}(c)\right\}
\end{aligned}
$$

For $\langle c, d\rangle,\left\langle c^{\prime}, d^{\prime}\right\rangle \in \mathbb{Q}_{\bar{X}}^{\dagger}$,

$$
\begin{aligned}
& \left\langle c^{\prime}, d^{\prime}\right\rangle \leq_{\mathbb{Q}_{\bar{X}}^{\dagger}}\langle c, d\rangle \Leftrightarrow \\
& c^{\prime} \leq_{\mathbb{C}_{\kappa}} c, \operatorname{dom}\left(d^{\prime}\right) \supseteq \operatorname{dom}(d), \\
& \forall \alpha \in \operatorname{dom}(d)\left(s^{d^{\prime}(\alpha)} \supseteq s^{d(\alpha)} \wedge F^{d^{\prime}(\alpha)} \supseteq F^{d(\alpha)} \wedge\right. \\
& \forall \xi \in F^{d(\alpha)} \cap X_{\alpha} \forall n \in \operatorname{dom}\left(s^{d^{\prime}(\alpha)}\right) \backslash \operatorname{dom}\left(s^{d(\alpha)}\right) \\
& \left.\left(c^{\prime}(\xi, n) \leq s^{d^{\prime}(\alpha)}(n)\right)\right) .
\end{aligned}
$$

The following can be shown easily by standard arguments:

Lemma 6.2. $\Phi: \mathbb{Q}_{\bar{X}}^{\dagger} \rightarrow \mathbb{Q}_{\bar{X}},\langle c, d\rangle \mapsto\langle c, \check{d}\rangle$, is a dense embedding of $\mathbb{Q}_{\bar{X}}^{\dagger}$ into $\mathbb{Q}_{\bar{X}}$.

$\mathbb{Q}_{\bar{X}}$ and $\mathbb{Q}_{\bar{X}}^{\dagger}$ are thus forcing equivalent.

For $p \in \mathbb{Q}_{\bar{X}}^{\dagger}$ with $p=\langle c, d\rangle$, let

$$
\begin{aligned}
& \operatorname{supp}_{0}(p)=\{\alpha<\kappa:\langle\alpha, n\rangle \in \operatorname{dom}(c) \text { for some } n \in \omega\}, \\
& \operatorname{supp}_{1}(p)=\operatorname{dom}(d) .
\end{aligned}
$$

For a $\mathbb{Q}_{\bar{X}}^{\dagger}$-name $\dot{a}, \operatorname{supp}_{0}(\dot{a})$ and $\operatorname{supp}_{1}(\dot{a})$ are also defined in analogy to (4.4).

In Theorem 6.4, we assume $\mathrm{CH}+(A)$ and let, for a structure $\left\langle\left(\omega_{2}\right)^{2}, A, \mathcal{F}\right\rangle$ as in (A), $\kappa=\omega_{2}$ and $\bar{X}=\left\langle X_{\alpha}: \alpha<\omega_{2}\right\rangle$ where $X_{\alpha}=\left\{\beta \in \omega_{2}:\langle\alpha, \beta\rangle \in A\right\}$ for $\alpha<\omega_{2}$. For such $\bar{X}$, the next lemma follows immediately from (6.3) and (6.4).

Lemma 6.3. Suppose that $\left\langle\left(\omega_{2}\right)^{2}, A, \mathcal{F}\right\rangle$ and $\bar{X}$ are as above. If $\langle\phi, \psi\rangle$ $\in \mathcal{F}$, then the mapping $j_{\langle\phi, \psi\rangle}: \mathbb{Q}_{\bar{X}}^{\dagger} \rightarrow \mathbb{Q}_{\bar{X}}^{\dagger}$ defined by

$$
j_{\langle\phi, \psi\rangle}(\langle c, d\rangle)=\left\langle c^{\prime}, d^{\prime}\right\rangle
$$

for $\langle c, d\rangle \in \mathbb{Q}_{\bar{X}}^{\dagger}$, where $c^{\prime}$ and $d^{\prime}$ are such that

$$
\begin{aligned}
& \operatorname{dom}\left(c^{\prime}\right)=\{\langle\phi(\alpha), n\rangle:\langle\alpha, n\rangle \in \operatorname{dom}(c)\} ; \\
& c^{\prime}(\langle\phi(\alpha), n\rangle)=c(\langle\alpha, n\rangle) \quad \text { for }\langle\alpha, n\rangle \in \operatorname{dom}(c) ; \\
& \operatorname{dom}\left(d^{\prime}\right)=\psi^{\prime \prime} \operatorname{dom}(d) ; \\
& F^{d^{\prime}(\psi(\xi))}=F^{d(\xi)} \quad \text { and } \quad s^{d^{\prime}(\psi(\xi))}=s^{d(\xi)} \quad \text { for } \xi \in \operatorname{dom}(d),
\end{aligned}
$$

is an automorphism of the poset $\mathbb{Q}_{\bar{X}}^{\dagger}$. 
Similarly to Section 4 , we shall also denote by $j_{\langle\phi, \psi\rangle}$ the corresponding mapping on $\mathbb{Q}_{\bar{X}}^{\dagger}$-names.

The following theorem together with the consistency result in Section 7 gives the consistency of the conjunction of the assertions $\mathfrak{c}=\mathfrak{b}^{*}=\aleph_{2}$, $\mathfrak{d o}=\aleph_{1}$ and $\neg \mathrm{C}^{\mathrm{S}}\left(\aleph_{2}\right)$ over ZFC.

Theorem 6.4. Assume $\mathrm{CH}$ and $(\mathrm{A})$. Let $\left\langle\left(\omega_{2}\right)^{2}, A, \mathcal{F}\right\rangle$ be a structure satisfying (6.1)-(6.5) and let $\bar{X}=\left\langle X_{\alpha}: \alpha<\omega_{2}\right\rangle$ where $X_{\alpha}=\left\{\beta \in \omega_{2}\right.$ : $\langle\alpha, \beta\rangle \in A\}$. Then $\Vdash_{\mathbb{Q}_{\bar{X}}^{\dagger}} " \mathfrak{c}=\mathfrak{b}^{*}=\aleph_{2} \wedge \mathfrak{d o}=\aleph_{1} \wedge \neg \mathrm{C}^{\mathrm{s}}\left(\aleph_{2}\right) "$.

Proof. First, we show that $\nVdash_{\mathbb{Q}_{\bar{X}}^{\dagger}}$ " $\mathfrak{c}=\mathfrak{b}^{*}=\aleph_{2}$ ". Let $G$ be a $\left(V, \mathbb{Q}_{\bar{X}}^{\dagger}\right)$ generic filter. Working in $V[G]$, let $\bar{f}=\left\langle c_{\alpha}: \alpha<\omega_{2}\right\rangle$ be the sequence of Cohen reals added by the $\mathbb{C}_{\omega_{2}}$ part of $\mathbb{Q}_{\bar{X}}$ and $d_{\alpha}$ be the Hechler type real added by $\mathbb{D}_{\bar{f}, X_{\alpha}}$ for $\alpha<\omega_{2}$. By (6.1), $\left\{c_{\alpha}: \alpha<\gamma\right\}$ is bounded by $d_{\gamma}$ for all $\gamma<\omega_{2}$. On the other hand, $\left\{c_{\alpha}: \alpha<\omega_{2}\right\}$ is unbounded by (6.2) and the c.c.c. of $\prod_{\alpha<\omega_{2}} \mathbb{D}_{\bar{f}, X_{\alpha}}$ (in $V[\bar{f}]$ ). This shows that $V[G] \models \aleph_{2} \leq \mathfrak{b}^{*}$. Since $\left|\mathbb{Q}_{\bar{X}}^{\dagger}\right|=\aleph_{2}$ by $\mathrm{CH}$, we have $V[G] \models \mathfrak{c} \leq \aleph_{2}$.

To show that $\mathbb{Q}_{\bar{X}}^{\dagger}$ forces $\mathfrak{d o}=\aleph_{1}$, suppose that $\dot{f}_{\alpha}, \alpha<\omega_{2}$, are $\mathbb{Q}_{\bar{X}}^{\dagger}$ names of elements of $\omega_{\omega}, \varphi(x, y, z)$ a formula in $\mathcal{L}_{\mathrm{ZF}}$, and $\dot{a}$ a $\mathbb{Q}_{\bar{X}}^{\dagger}$-name of an element of $\omega_{\omega}$ such that

$$
\Vdash_{\mathbb{Q}_{\bar{X}}^{\dagger}} \text { " } \mathcal{H}\left(\aleph_{1}\right) \models \varphi\left(\dot{f}_{\alpha}, \dot{f}_{\beta}, \dot{a}\right) " \quad \text { for all } \alpha<\beta<\omega_{2} .
$$

By the maximal principle, it is enough to show that there are $\eta_{1}<\eta_{0}<\omega_{2}$ such that

$$
\Vdash_{\mathbb{Q}_{\bar{X}}^{\dagger}} " \mathcal{H}\left(\aleph_{1}\right) \models \varphi\left(\dot{f}_{\eta_{0}}, \dot{f}_{\eta_{1}}, \dot{a}\right) " .
$$

For $\xi<\omega_{2}$, let

$$
A_{\xi}=\operatorname{supp}_{1}\left(\dot{f}_{\xi}\right) \cup \operatorname{supp}_{1}(\dot{a}), \quad B_{\xi}=\operatorname{supp}_{0}\left(\dot{f}_{\xi}\right) \cup \operatorname{supp}_{0}(\dot{a}) .
$$

By $\mathrm{CH}$, the $\Delta$-system lemma and (6.5), we can find a stationary $S \subseteq E_{\omega_{1}}^{\omega_{2}}$ such that

(6.12) $A_{\xi}, \xi \in S$, form a $\Delta$-system whose root is an initial segment of each of $A_{\xi}, \xi \in S ; B_{\xi}, \xi \in S$, form a $\Delta$-system such that its root is an initial segment of each of $B_{\xi}, \xi \in S$;

(6.13) for any distinct $\zeta_{0}, \zeta_{1}, \eta_{0}, \eta_{1} \in S$, there is $\langle\phi, \psi\rangle \in \mathcal{F}$ such that

(a) $\phi^{\prime \prime} A_{\zeta_{i}}=A_{\eta_{i}}, \psi^{\prime \prime} B_{\zeta_{i}}=B_{\eta_{i}}$;

(b) $\phi\left\lceil A_{\zeta_{i}}: A_{\zeta_{i}} \rightarrow A_{\eta_{i}}\right.$ and $\psi \uparrow B_{\zeta_{i}}: B_{\zeta_{i}} \rightarrow B_{\eta_{i}}$ are order isomorphisms for $i \in 2$;

(6.14) $j_{\langle\phi, \psi\rangle}\left(\dot{f}_{\zeta}\right)=\dot{f}_{\eta}$ for any distinct $\zeta, \eta \in S$ and $\langle\phi, \psi\rangle \in \mathcal{F}$ as in (6.13) with $\zeta_{0}=\zeta$ and $\eta_{0}=\eta$. 
Note that, by (6.12) and (6.13)(b), we have

$$
j_{\langle\phi, \psi\rangle}(\dot{a})=\dot{a}
$$

for any $\langle\phi, \psi\rangle$ as in (6.13).

Now, let $\zeta_{0}, \zeta_{1}, \eta_{0}, \eta_{1} \in S$ be four distinct elements of $S$ such that $\zeta_{0}<\zeta_{1}$ and $\eta_{1}<\eta_{0}$. By (6.11), we have

$$
\Vdash_{\mathbb{Q}_{\bar{X}}^{\dagger}} " \mathcal{H}\left(\aleph_{1}\right) \models \varphi\left(\dot{f}_{\zeta_{0}}, \dot{f}_{\zeta_{1}}, \dot{a}\right) " .
$$

Hence, by mapping this situation via $j_{\langle\phi, \psi\rangle}$ for $\langle\phi, \psi\rangle \in \mathcal{F}$ as in (6.13) for these $\zeta_{0}, \zeta_{1}, \eta_{0}, \eta_{1}$, we obtain

$$
\Vdash_{\mathbb{Q}_{\bar{X}}^{\dagger}} " \mathcal{H}\left(\aleph_{1}\right) \models \varphi\left(\dot{f}_{\eta_{0}}, \dot{f}_{\eta_{1}}, \dot{a}\right) " .
$$

Thus, $\eta_{0}, \eta_{1}$ above are as desired.

Finally, we show that $\mathbb{Q}_{\bar{X}}^{\dagger}$ forces the negation of $\mathrm{C}^{\mathrm{S}}\left(\aleph_{2}\right)$.

Let $\left\langle r_{n}^{0}, s_{n}^{0}, r_{n}^{1}, s_{n}^{1}\right\rangle, n \in \omega$, list all quadruples of finite sequences $r^{0}, s^{0}$, $r^{1}, s^{1} \in \omega>\omega$ such that

$$
\begin{aligned}
\left|r^{0}\right| & =\left|s^{0}\right|=\left|r^{1}\right|=\left|s^{1}\right|, \\
\left\langle r^{0}, s^{0}\right\rangle & \neq\left\langle r^{1}, s^{1}\right\rangle \quad \text { if }\left|r^{0}\right|>0 .
\end{aligned}
$$

We further assume that the enumeration $\left\langle\left\langle r_{n}^{0}, s_{n}^{0}, r_{n}^{1}, s_{n}^{1}\right\rangle: n \in \omega\right\rangle$ is arranged so that

$$
\left|r_{n}^{0}\right| \leq n \quad \text { for all } n \in \omega .
$$

Now, working in $V[G]$, let $a_{\alpha}, \alpha<\omega_{2}$, be the subsets of $\omega$ defined by: $n \in a_{\alpha} \Leftrightarrow$ one of (6.19) and (6.20) below holds:

$$
\begin{aligned}
& r_{n}^{0} \subseteq c_{\alpha}, s_{n}^{0} \subseteq d_{\alpha+1}, \\
& c_{\alpha}(n)=0, d_{\alpha+1}(n)=1, c_{\alpha}(n+1)=2 \text { and } d_{\alpha+1}(n+1)=3 ; \\
& r_{n}^{1} \subseteq c_{\alpha}, s_{n}^{1} \subseteq d_{\alpha+1}, \\
& c_{\alpha}(n)=2, d_{\alpha+1}(n)=3, c_{\alpha}(n+1)=0 \text { and } d_{\alpha+1}(n+1)=1 .
\end{aligned}
$$

Let

$$
a_{\alpha, n}=a_{\alpha} \backslash\left\{k:\left|r_{k}^{0}\right|<n\right\} \quad \text { for } \alpha<\omega_{2} \text { and } n \in \omega .
$$

We show that the matrix $\left\langle a_{\alpha, n}: \alpha<\omega_{2}, n \in \omega\right\rangle$ together with $T={ }^{2} \omega$ is a counter-example to $\mathrm{C}^{\mathrm{s}}\left(\aleph_{2}\right)$. For this, it is enough to prove the following:

Claim 6.4.1. If $S_{0}, S_{1}$ are cofinal subsets of $\omega_{2}$, then

(1) there exist $n<\omega, \alpha \in S_{0}$ and $\beta \in S_{1}$ such that $a_{\alpha, n} \cap a_{\beta, n}=\emptyset$;

(2) for any $t \in{ }^{2} \omega$, there are $\alpha \in S_{0}$ and $\beta \in S_{1}$ such that $a_{\alpha, t(0)} \cap a_{\beta, t(1)} \neq \emptyset$.

$\vdash$ Working in the ground model, let $\dot{S}_{0}$ and $\dot{S}_{1}$ be $\mathbb{Q}_{\bar{X}}^{\dagger}$-names for the cofinal subsets of $\omega_{2}$. Let $p \in \mathbb{Q}_{\bar{X}}^{\dagger}$. For $\alpha<\omega_{2}$, let $p_{\alpha} \in \mathbb{Q}_{\bar{X}}^{\dagger}$ and $\gamma_{\alpha}, \delta_{\alpha} \in \omega_{2}$ be such that

$$
\gamma_{\alpha}<\delta_{\alpha}<\gamma_{\beta}<\delta_{\beta} \text { for all } \alpha<\beta<\omega_{2}
$$


(6.23) $p_{\alpha} \leq_{\mathbb{Q}_{\bar{X}}^{\dagger}} p, p_{\alpha}=\left\langle c^{\alpha}, d^{\alpha}\right\rangle$ for all $\alpha<\omega_{2}$;

(6.24) $p_{\alpha} \Vdash_{\mathbb{Q}_{\bar{X}}^{\dagger}} " \gamma_{\alpha} \in \dot{S}_{0}, \delta_{\alpha} \in \dot{S}_{1} "$.

By the $\Delta$-system lemma, we find a stationary $U \subseteq E_{\omega_{1}}^{\omega_{2}}$ and $A_{\alpha}, B_{\alpha} \in\left[\omega_{2}\right]^{<\aleph_{0}}$ for $\alpha \in U$ such that

(6.25) $\operatorname{supp}_{0}\left(p_{\alpha}\right) \subseteq A_{\alpha}, \operatorname{supp}_{1}\left(p_{\alpha}\right) \subseteq B_{\alpha}$;

(6.26) $A_{\alpha}, \alpha \in U$, form a $\Delta$-system with root $A$, and $B_{\alpha}, \alpha \in U$, form a $\Delta$-system with root $B$;

$$
\gamma_{\alpha}, \gamma_{\alpha}+1, \delta_{\alpha}, \delta_{\alpha}+1 \in\left(A_{\alpha} \cap B_{\alpha}\right) \backslash(A \cup B)
$$

By thinning out $U$ further if necessary, we may also assume that there are some $k^{*}, n^{*} \in \omega$ such that

(6.28) $\operatorname{dom}\left(c^{\alpha}\right)=\operatorname{supp}_{0}\left(p_{\alpha}\right) \times k^{*}, \operatorname{dom}\left(s^{d^{\alpha}(\xi)}\right)=k^{*}$ for all $\xi \in \operatorname{supp}_{1}\left(p_{\alpha}\right)$;

(6.29) $\quad c^{\alpha}\left(\gamma_{\alpha}, \cdot\right)=r_{n^{*}}^{0}, s^{d^{\alpha}\left(\gamma_{\alpha}+1\right)}=s_{n^{*}}^{0}$;

(6.30) $\quad c^{\alpha}\left(\delta_{\alpha}, \cdot\right)=r_{n^{*}}^{1}, s^{d^{\alpha}\left(\delta_{\alpha}+1\right)}=s_{n^{*}}^{1}$.

Without loss of generality, we may also assume that, for some fixed $c^{*}, d^{*}$,

$$
c^{\alpha} \uparrow A \times k^{*}=c^{*} \text { and }\left\langle s^{d^{\alpha}(\eta)}: \eta \in B\right\rangle=d^{*} \text { for all } \alpha \in U .
$$

Note that $p_{\alpha}, \alpha \in U$, are compatible by (6.25), (6.26) and (6.31).

Now, since $\dot{S}_{0}, \dot{S}_{1}, p$ were arbitrary, Claim $6.4 .1(1)$ is proved by the following subclaim:

SubCLAIm 6.4.1.1. For any $\alpha, \beta \in U$ with $\alpha<\beta$, there is $q \leq_{\mathbb{Q}_{\bar{X}}^{\dagger}} p$ such that

$$
q \Vdash_{\mathbb{Q}_{\bar{X}}^{\dagger}} " \gamma_{\alpha} \in \dot{S}_{0}, \delta_{\beta} \in \dot{S}_{1}, \dot{a}_{\gamma_{\alpha}, n^{*}} \cap \dot{a}_{\delta_{\beta}, n^{*}}=\emptyset " .
$$

$\vdash$ Let $q=\left\langle c^{q}, d^{q}\right\rangle$ be the common extension of $p_{\alpha}$ and $p_{\beta}$ such that

$$
\begin{gathered}
\gamma_{\alpha} \in F^{d^{q}\left(\delta_{\beta}+1\right)}, \\
\operatorname{dom}\left(s^{d^{q}(\xi)}\right)=k^{*} \quad \text { for all } \xi \in \operatorname{dom}\left(d^{q}\right) .
\end{gathered}
$$

Let $G$ be a $\left(V, \mathbb{Q}_{\bar{X}}^{\dagger}\right)$-generic filter with $q \in G$. In $V[G]$, we have

$$
c_{\gamma_{\alpha}}(m) \leq d_{\delta_{\beta}+1}(m) \quad \text { for all } m \geq k^{*}
$$

by $(6.28),(6.32)$ and $(6.33)$.

Now, toward a contradiction, assume that $a_{\gamma_{\alpha}, n^{*}} \cap a_{\delta_{\beta}, n^{*}} \neq \emptyset$ and let $m \in a_{\gamma_{\alpha}, n^{*}} \cap a_{\delta_{\beta}, n^{*}}$. By the definition of $a_{\alpha}$ 's it follows that, for some $i$, $j \in 2$, we have

$$
r_{m}^{i} \subseteq c_{\gamma_{\alpha}}, \quad s_{m}^{i} \subseteq d_{\gamma_{\alpha}+1} ; \quad r_{m}^{j} \subseteq c_{\delta_{\beta}}, \quad s_{m}^{j} \subseteq d_{\delta_{\beta}+1} .
$$

On the other hand, since $q \in G$, we have $p_{\alpha}, p_{\beta} \in G$. It follows that

$$
r_{n^{*}}^{0} \subseteq c_{\gamma_{\alpha}}, \quad s_{n^{*}}^{0} \subseteq d_{\gamma_{\alpha}+1} ; \quad r_{n^{*}}^{1} \subseteq c_{\delta_{\beta}}, \quad s_{n^{*}}^{1} \subseteq d_{\delta_{\beta}+1}
$$


by (6.29) and (6.30). By the definition (6.21) of $a_{\gamma_{\alpha}, n}$ 's, we have $\left|r_{m}^{0}\right| \geq n^{*}$. Thus we have, either

$r_{n^{*}}^{0} \subseteq r_{m}^{0} \subseteq c_{\gamma_{\alpha}}, \quad s_{n^{*}}^{0} \subseteq s_{m}^{0} \subseteq d_{\gamma_{\alpha}+1} ; \quad r_{n^{*}}^{1} \subseteq r_{m}^{1} \subseteq c_{\gamma_{\beta}}, \quad s_{n^{*}}^{1} \subseteq s_{m}^{1} \subseteq d_{\gamma_{\beta}+1} ;$

or

$r_{n^{*}}^{0} \subseteq r_{m}^{1} \subseteq c_{\gamma_{\alpha}}, \quad s_{n^{*}}^{0} \subseteq s_{m}^{1} \subseteq d_{\gamma_{\alpha}+1} ; \quad r_{n^{*}}^{1} \subseteq r_{m}^{0} \subseteq c_{\gamma_{\beta}}, \quad s_{n^{*}}^{1} \subseteq s_{m}^{0} \subseteq d_{\gamma_{\beta}+1}$.

In the first case, we must have $c_{\gamma_{\alpha}}(m+1)=2$ and $d_{\delta_{\beta}+1}(m+1)=1$ by $(6.19)$ and (6.20). This contradicts (6.34). Similarly, in the second case, $c_{\gamma_{\alpha}}(m)=2$ and $d_{\delta_{\beta}+1}(m)=1$. This again contradicts (6.34). $\dashv$ (Subclaim 6.4.1.1)

(2) of Claim 6.4.1 follows from the next subclaim:

SubClaim 6.4.1.2. For any $t \in{ }^{2} \omega$ and $\alpha, \beta \in U$ with $\alpha<\beta$, there is $q \leq_{\mathbb{Q}_{\bar{X}}^{\dagger}}$ p such that

$$
q \Vdash_{\mathbb{Q}_{\bar{X}}^{\dagger}} " \gamma_{\alpha} \in \dot{S}_{0}, \delta_{\beta} \in \dot{S}_{1}, \dot{a}_{\gamma(\alpha), t(0)} \cap \dot{a}_{\delta(\beta), t(1)} \neq \emptyset " .
$$

$\vdash$ For each $\xi \in\{\alpha, \beta\}$, let $\tilde{p}_{\xi} \leq_{\mathbb{Q}_{\bar{X}}^{\dagger}} p_{\xi}$ with $\tilde{p}_{\xi}=\left\langle\tilde{c}^{\xi}, \tilde{d}^{\xi}\right\rangle$ and $m \in \omega$ be such that

$$
\begin{aligned}
& \tilde{c}^{\xi}\left(\gamma_{\xi}, \cdot\right)=r_{m}^{0}, s^{\tilde{d}^{\xi}\left(\gamma_{\xi}+1\right)}=s_{m}^{0} ; \tilde{c}^{\xi}\left(\delta_{\xi}, \cdot\right)=r_{m}^{1}, s^{\tilde{d}^{\xi}\left(\delta_{\xi}+1\right)}=s_{m}^{1} ; \\
& \left|r_{m}^{0}\right| \geq t(0), t(1) \\
& \tilde{c}^{\xi}\left\lceil A \times \omega=c^{\xi}\left\lceil A \times \omega \text { and }\left\langle s^{\tilde{d}^{\xi}(\eta)}: \eta \in B\right\rangle=\left\langle s^{d^{\xi}(\eta)}: \eta \in B\right\rangle\right. \text {. }\right.
\end{aligned}
$$

Let $q^{0}=\left\langle c^{q^{0}}, d^{q^{0}}\right\rangle$ be the maximal (with respect to $\leq_{\mathbb{Q}_{\bar{X}}^{\dagger}}$ ) common extension of $\tilde{p}_{\alpha}$ and $\tilde{p}_{\beta}$ which exists because of (6.37) and (6.38). Extend $q^{0}$ further to $q=\left\langle c^{q}, d^{q}\right\rangle$ such that

$$
\begin{aligned}
& \left|c^{q}\left(\gamma_{\alpha}, \cdot\right)\right|=\left|c^{q}\left(\delta_{\beta}, \cdot\right)\right|=\left|s^{d^{q}\left(\gamma_{\alpha}+1\right)}\right|=\left|s^{d^{q}\left(\delta_{\beta}+1\right)}\right|=m+2 \\
& c^{q}\left(\gamma_{\alpha}, m\right)=0, s^{d^{q}\left(\gamma_{\alpha}+1\right)}(m)=1 \\
& c^{q}\left(\gamma_{\alpha}, m+1\right)=2, s^{d^{q}\left(\gamma_{\alpha}+1\right)}(m+1)=3 \\
& c^{q}\left(\delta_{\beta}, m\right)=2, s^{d^{q}\left(\delta_{\beta}+1\right)}(m)=3 \\
& c^{q}\left(\delta_{\beta}, m+1\right)=0, s^{d^{q}\left(\delta_{\beta}+1\right)}(m+1)=1 .
\end{aligned}
$$

This is possible because $\gamma_{\alpha} \notin F^{d^{q^{0}}\left(\delta_{\beta}+1\right)}$ and $\delta_{\beta} \notin F^{d^{q^{0}}\left(\gamma_{\alpha}+1\right)}$ by the maximality of $q^{0}$ and (6.37).

By (6.35), (6.40), (6.41), by the definition (6.21) of $a_{\alpha, n}$ 's, and since $\left|r_{m}^{0}\right| \geq t(0), t(1)$, we have $q \Vdash_{\mathbb{Q}_{\bar{X}}^{\dagger}} " m \in \dot{a}_{\gamma_{\alpha}, t(0)} \cap \dot{a}_{\delta_{\beta}, t(1)}$ ". Since $q \leq_{\mathbb{Q}_{\bar{X}}^{\dagger}}$ $p_{\alpha}, p_{\beta}$, we also have $q \Vdash_{\mathbb{Q}_{\bar{X}}^{\dagger}}$ " $\gamma_{\alpha} \in \dot{S}_{0}, \delta_{\beta} \in \dot{S}_{1}$ ". Thus, $q$ as above is as desired. $\dashv$ (Subclaim 6.4.1.2) $\dashv$ (Claim 6.4.1) • (Theorem 6.4) 
Note that in the proof of $\neg \mathrm{C}^{\mathrm{S}}\left(\aleph_{2}\right)$ in Theorem 6.4, we used only (6.1) from the assumption (A). Note also that this proof actually shows that in the generic extension the negation of $\mathrm{C}\left(\aleph_{2}\right)$ from [13] holds which is a weakening of $\mathrm{C}^{\mathrm{S}}\left(\aleph_{2}\right)$ obtained by replacing the "stationary" condition in the formulation of $\mathrm{C}^{\mathrm{S}}\left(\aleph_{2}\right)$ by "cofinal".

7. Forcing $\mathbf{C H}+(\mathbf{A})$. In this section, we define under $\mathrm{CH}$ a $\sigma$-closed $\aleph_{2}$-c.c. poset $\mathbb{P}_{0}$ which forces the combinatorial assertion $(\mathrm{A})$ of the previous section. The poset $\mathbb{P}_{0}$ is defined as follows:

$$
p \in \mathbb{P}_{0} \Leftrightarrow p=\left\langle X^{p}, Y^{p}, \tau^{p},\left\langle\phi_{\xi}^{p}, \psi_{\xi}^{p}: \xi \in D^{p}\right\rangle\right\rangle
$$

where

$$
\begin{aligned}
& X^{p}, Y^{p} \in\left[\omega_{2}\right]^{\aleph_{0}} \\
& D^{p} \in\left[\omega_{2}\right]^{\aleph_{0}}
\end{aligned}
$$

(7.3) for all $\xi \in D^{p}, \phi_{\xi}^{p}: X^{p} \rightarrow X^{p}$ and $\psi_{\xi}^{p}: Y^{p} \rightarrow Y^{p}$ are involutions (that is, bijections $\phi$ such that $\phi^{-1}=\phi$ );

(7.4) for all $\xi \in D^{p}, \alpha \in X^{p}$ and $\beta \in Y^{p}$,

(a) $\phi_{\xi}^{p}(\alpha)<\alpha+\xi+\omega_{1}$,

(b) $\psi_{\xi}^{p}(\beta)<\beta+\xi+\omega_{1}$.

Note that we also have $\alpha<\phi_{\xi}^{p}(\alpha)+\xi+\omega_{1}$ and $\alpha<\psi_{\xi}^{p}(\beta)+\xi+\omega_{1}$ for all $\xi \in D^{p}, \alpha \in X^{p}$ and $\beta \in Y^{p}$ since $\phi_{\xi}^{p}$ and $\psi_{\xi}^{p}$ are involutions by (7.3).

(7.5) $\quad \tau^{p}: X^{p} \times Y^{p} \rightarrow 2$;

(7.6) $\tau^{p}(\alpha, \beta)=\tau^{p}\left(\phi_{\xi}^{p}(\alpha), \psi_{\xi}^{p}(\beta)\right)$ for all $\xi \in D^{p}, \alpha \in X^{p}$ and $\beta \in Y^{p}$;

(7.7) $\tau^{p}(\alpha, \beta)=1$ for all $\langle\alpha, \beta\rangle \in X^{p} \times Y^{p}$ with $\beta<\alpha$.

The ordering on $\mathbb{P}_{0}$ is defined as follows: For $p, q \in \mathbb{P}_{0}$ with

$$
p=\left\langle X^{p}, Y^{p}, \tau^{p},\left\langle\phi_{\xi}^{p}, \psi_{\xi}^{p}: \xi \in D^{p}\right\rangle\right\rangle, \quad q=\left\langle X^{q}, Y^{q}, \tau^{q},\left\langle\phi_{\xi}^{q}, \psi_{\xi}^{q}: \xi \in D^{q}\right\rangle\right\rangle,
$$

we have:

$$
\begin{aligned}
p \leq_{\mathbb{P}_{0}} q \Leftrightarrow & \text { (a) } X^{p} \supseteq X^{q}, Y^{p} \supseteq Y^{q} \\
& \text { (b) } D^{p} \supseteq D^{q} ; \\
& \text { (c) } \phi_{\xi}^{p} \supseteq \phi_{\xi}^{q} \text { and } \psi_{\xi}^{p} \supseteq \psi_{\xi}^{q} \text { for all } \xi \in D^{q} ; \\
& \text { (d) } \tau^{p} \supseteq \tau^{q} ; \\
& \text { (e) } \tau^{p} \uparrow\left(X^{p} \backslash X^{q}\right) \times Y^{q} \equiv 1 .
\end{aligned}
$$

For $p \in \mathbb{P}_{0}$ with $p=\left\langle X^{p}, Y^{p}, \tau^{p},\left\langle\phi_{\xi}^{p}, \psi_{\xi}^{p}: \xi \in D^{p}\right\rangle\right\rangle$, we intend to approximate the characteristic function of the set $A$ in the assertion (A) by $\tau^{p}$. More precisely, in a generic extension $V[G]$ for a $\left(V, \mathbb{P}_{0}\right)$-generic $G$, letting 


$$
\tau=\bigcup_{p \in G} \tau^{p} ; \quad \phi_{\xi}=\bigcup_{p \in G} \phi_{\xi}^{p} \text { and } \psi_{\xi}=\bigcup_{p \in G} \psi_{\xi}^{p} \text { for } \xi \in \omega_{2} ;
$$

$$
A=\tau^{-1 "}\{1\} \text { and } \mathcal{F}=\left\{\left\langle\phi_{\xi}, \psi_{\xi}\right\rangle: \xi \in \omega_{2}\right\},
$$

we are aiming to force $\left\langle\left(\omega_{2}\right)^{2}, A, \mathcal{F}\right\rangle$ to satisfy $(6.1)-(6.5)$ in (A).

Of the conditions in the definition of $\mathbb{P}_{0},(7.5)$ and $(7.8)(\mathrm{d})$ force $\tau$ to be a function. Furthermore, $\tau: \omega_{2} \times \omega_{2} \rightarrow 2$ by a density argument and Lemma 7.1(a).

(7.3) and (7.8)(c) make $\phi_{\xi}$ and $\psi_{\xi}$ mappings for all $\xi \in \omega_{2}$; they are forced to be involutions on $\omega_{2}$ by $(7.3)$ and Lemma 7.1(a). Thus $\left\langle\left(\omega_{2}\right)^{2}, A, \mathcal{F}\right\rangle$ is forced to satisfy (6.3).

By (7.7) (and Lemma 7.1(a)), $\left\langle\left(\omega_{2}\right)^{2}, A, \mathcal{F}\right\rangle$ is forced to satisfy the second inclusion of (6.1).

By (7.6), $\left\langle\left(\omega_{2}\right)^{2}, A, \mathcal{F}\right\rangle$ is forced to satisfy (6.4).

$(7.4)$ and $(7.8)(\mathrm{e})$ are technical conditions whose role will be clear later in the course of the proof.

By the definition of $\mathbb{P}_{0}$, it is clear that $\mathbb{P}_{0}$ is $\sigma$-closed. Thus, we will be done by showing that $\mathbb{P}_{0}$ satisfies the $\aleph_{2}$-c.c. and it forces that $\left\langle\left(\omega_{2}\right)^{2}, A, \mathcal{F}\right\rangle$ as above satisfies the conditions (6.2) and (6.5).

The next lemma follows readily from the definition of $\mathbb{P}_{0}$.

LEMMA 7.1.

(a) For any $\alpha, \beta<\omega_{2}$, the set

$$
\begin{gathered}
D_{\alpha, \beta}=\left\{p \in \mathbb{P}_{0}: p=\left\langle X^{p}, Y^{p}, \tau^{p},\left\langle\phi_{\xi}^{p}, \psi_{\xi}^{p}: \xi \in D^{p}\right\rangle\right\rangle,\right. \\
\left.\alpha \in X^{p} \text { and } \beta \in Y^{p}\right\}
\end{gathered}
$$

is dense in $\mathbb{P}_{0}$.

(b) For any $C \in\left[\omega_{2}\right]^{\aleph_{0}}$ and any $\beta<\omega_{2}$,

$$
\begin{aligned}
E_{C, \beta}=\left\{p \in \mathbb{P}_{0}:\right. & p=\left\langle X^{p}, Y^{p}, \tau^{p},\left\langle\phi_{\xi}^{p}, \psi_{\xi}^{p}: \xi \in D^{p}\right\rangle\right\rangle, \\
& C \subseteq X^{p} \text { and for some } \delta \in Y^{p} \text { with } \delta \geq \beta, \\
& \left.\tau^{p}(\gamma, \delta)=0 \text { for all } \gamma \in C\right\}
\end{aligned}
$$

is dense in $\mathbb{P}_{0}$.

In the rest of the section, we are going to work mainly in the ground model (where CH holds). Let $\dot{\tau}, \dot{\phi}_{\xi}, \dot{\psi}_{\xi}$ for $\xi \in \omega_{2}, \dot{A}$ and $\dot{\mathcal{F}}$ be $\mathbb{P}_{0}$-names of $\tau, \phi_{\xi}, \psi_{\xi}$ for $\xi \in \omega_{2}, A$ and $\mathcal{F}$ as above, respectively.

Lemma 7.2. $\|_{\mathbb{P}_{0}}$ “ $\left\langle\left(\omega_{2}\right)^{2}, \dot{A}, \dot{\mathcal{F}}\right\rangle \models(6.2)$ ".

Proof. By a density argument with Lemma 7.1(b). - (Lemma 7.2)

For $\xi<\omega_{2}, X, X^{\prime}, Y, Y^{\prime} \in\left[\omega_{2}\right]^{\aleph_{0}}$ with $X^{\prime} \subseteq X$ and $Y^{\prime} \subseteq Y, \tau: X \times Y \rightarrow 2$ and involutions $\phi^{\prime}: X^{\prime} \rightarrow X^{\prime}, \psi: Y^{\prime} \rightarrow Y^{\prime}$, let us call the quintuple $\left\langle X, Y, \tau, \phi^{\prime}, \psi^{\prime}\right\rangle$ a $\xi$-extendable semi-condition if 
(7.11) $\phi^{\prime}(\alpha)<\alpha+\xi+\omega_{1}$ and $\psi^{\prime}(\beta)<\beta+\xi+\omega_{1}$ for all $\alpha \in X^{\prime}$ and $\beta \in Y^{\prime}$;

(7.12) $\tau(\alpha, \beta)=\tau(\phi(\alpha), \psi(\beta))$ for all $\alpha \in X^{\prime}$ and $\beta \in Y^{\prime}$;

(7.13) $\tau(\alpha, \beta)=1$ for all $\alpha \in X$ and $\beta \in Y$ with $\beta<\alpha$;

(7.14) $\tau \uparrow\left(\left(X \backslash X^{\prime}\right) \times Y^{\prime}\right) \equiv 1$.

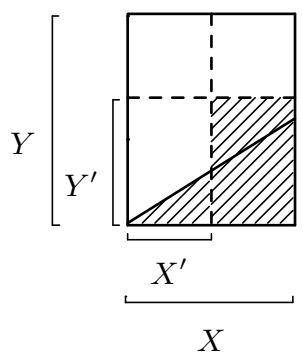

Fig. 6

Note that the sets $X^{\prime}$ and $Y^{\prime}$, though not mentioned explicitly in the definition of $\xi$-extendable semi-condition, can be recovered from $\phi^{\prime}$ and $\psi^{\prime}$.

Note also that (7.14) holds vacuously if $X=X^{\prime}$. Hence, if $p \in \mathbb{P}_{0}$ with $p=\left\langle X^{p}, Y^{p}, \tau^{p},\left\langle\phi_{\xi}^{p}, \psi_{\xi}^{p}: \xi \in D^{p}\right\rangle\right\rangle$, then the quintuple $\left\langle X^{p}, Y^{p}, \tau^{p}, \phi_{\xi}, \psi_{\xi}\right\rangle$ is a $\xi$-extendable semi-condition for all $\xi \in D^{p}$.

The following two lemmas explain the choice of the naming of $\xi$-extendable semi-conditions.

Lemma 7.3. For any $\xi<\omega_{2}, X, X^{\prime}, Y, Y^{\prime} \in\left[\omega_{2}\right]^{\aleph_{0}}$ with $X^{\prime} \subseteq X$ and $Y^{\prime} \subseteq Y, \tau: X \times Y \rightarrow 2$ as well as involutions $\phi^{\prime}: X^{\prime} \rightarrow X^{\prime}, \psi^{\prime}: Y^{\prime} \rightarrow Y^{\prime}$, if $\left\langle X, Y, \tau, \phi^{\prime}, \psi^{\prime}\right\rangle$ is a $\xi$-extendable semi-condition then there are $\tilde{X} \supseteq X$, $\tilde{Y} \supseteq Y, \tilde{\tau}: \tilde{X} \times \tilde{Y} \rightarrow 2$ with $\tilde{\tau} \supseteq \tau$ and involutions $\tilde{\phi}: \tilde{X} \rightarrow \tilde{X}, \tilde{\psi}: \tilde{Y} \rightarrow \tilde{Y}$ extending $\phi^{\prime}$ and $\psi^{\prime}$ respectively such that $\langle\tilde{X}, \tilde{Y}, \tilde{\tau}, \tilde{\phi}, \tilde{\psi}\rangle$ is a $\xi$-extendable semi-condition and

$$
\tilde{\tau} \uparrow((\tilde{X} \backslash X) \times Y) \equiv 1
$$

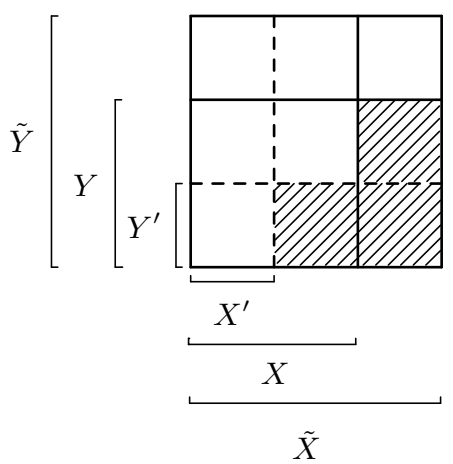

Fig. 7 
Proof. Let $X_{0} \in\left[\omega_{2} \backslash X\right]^{\leq \aleph_{0}}$ be such that

(7.16) $\quad X_{0}$ is order-isomorphic to $X \backslash X^{\prime}$ and the order-isomorphism identifies points of distance less than $\omega_{1}$ (that is, if $\alpha \in X \backslash X^{\prime}$ and $\alpha_{0} \in X_{0}$ are identified then $\alpha<\alpha_{0}+\omega_{1}$ and $\left.\alpha_{0}<\alpha+\omega_{1}\right)$.

Since $X$ (and hence also $X \backslash X^{\prime}$ ) is countable, we can easily choose the elements of $X_{0}$ recursively in otp $\left(X \backslash X^{\prime}\right)$ steps in accordance with (7.16). Put $\tilde{X}=X \dot{\cup} X_{0}$ and let $\tilde{\phi}$ be the extension of $\phi$ which maps $X_{0}$ orderisomorphically to $X \backslash X^{\prime}$ and vice versa. Fix $\theta<\omega_{1}$ such that

$$
\tilde{\phi}(\alpha) \leq \alpha+\xi+\theta \quad \text { for all } \alpha \in \tilde{X} .
$$

There is such a $\theta$ by (7.11), (7.16) and since $|\tilde{X}| \leq \aleph_{0}$.

Let $Y_{0} \in\left[\omega_{2} \backslash Y\right]^{\leq \aleph_{0}}$ be such that

(7.18) $\quad Y_{0}$ is order-isomorphic to $Y \backslash Y^{\prime}$ and the order-isomorphism identifies points of distance less than $\xi+\omega_{1}$;

(7.19) if $\beta \in Y \backslash Y^{\prime}$ and $\beta_{0} \in Y_{0}$ are identified then $\beta_{0}>\beta+\xi+\theta$.

It is easy to see that the elements of such a $Y_{0}$ can be chosen recursively in $\operatorname{otp}\left(Y \backslash Y^{\prime}\right)$ steps.

Now let $\tilde{Y}=Y \dot{\cup} Y_{0}$ and let $\tilde{\psi}$ be the extension of $\psi^{\prime}$ which maps $Y_{0}$ order-isomorphically to $Y \backslash Y^{\prime}$ and vice versa. Finally, define $\tilde{\tau}: \tilde{X} \times \tilde{Y} \rightarrow 2$ by

$$
\tilde{\tau}(\alpha, \beta)= \begin{cases}\tau(\alpha, \beta) & \text { if }\langle\alpha, \beta\rangle \in X \times Y, \\ \tau(\tilde{\phi}(\alpha), \tilde{\psi}(\beta)) & \text { if }\langle\alpha, \beta\rangle \in\left(X^{\prime} \times Y_{0}\right) \cup\left(X_{0} \times Y^{\prime}\right) \\ & \quad \cup\left(X_{0} \times Y_{0}\right), \\ 1 & \text { otherwise, }\end{cases}
$$

for every $\alpha \in \tilde{X}$ and $\beta \in \tilde{Y}$.

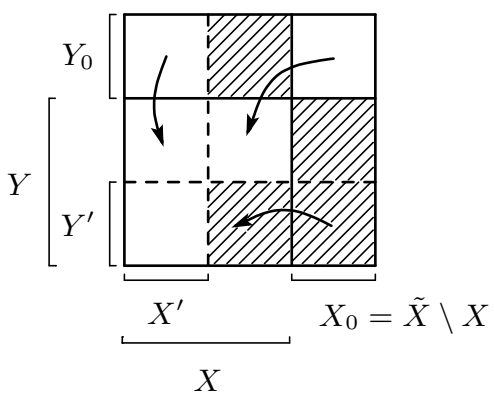

Fig. 8 
$\langle\tilde{X}, \tilde{Y}, \tilde{\tau}, \tilde{\phi}, \tilde{\psi}\rangle$ satisfies (7.11) by (7.17) and (7.18). It satisfies (7.12) by (7.20). Thus we will be done by checking that $\langle\tilde{X}, \tilde{Y}, \tilde{\tau}, \tilde{\phi}, \tilde{\psi}\rangle$ also satisfies (7.15) and (7.13).

For (7.15), suppose $\langle\alpha, \beta\rangle \in(\tilde{X} \backslash X) \times Y\left(=X_{0} \times Y\right)$. If $\langle\alpha, \beta\rangle \in X_{0} \times Y^{\prime}$ then $\tilde{\tau}(\alpha, \beta)=\tau(\tilde{\phi}(\alpha), \tilde{\psi}(\beta))$ by $(7.20)$. But $\langle\tilde{\phi}(\alpha), \tilde{\psi}(\beta)\rangle \in\left(X \backslash X^{\prime}\right) \times Y^{\prime}$ by definition of $\tilde{\phi}$ and $\tilde{\psi}$. Hence, by $(7.14), \tilde{\tau}(\alpha, \beta)=\tau(\tilde{\phi}(\alpha), \tilde{\psi}(\beta))=1$. If $\langle\alpha, \beta\rangle \in X_{0} \times\left(Y \backslash Y^{\prime}\right)$ then $\tilde{\tau}(\alpha, \beta)=1$ by the "otherwise" clause of (7.20).

For (7.13), it is enough to check that $\tilde{\tau}(\alpha, \beta)=1$ for $\langle\alpha, \beta\rangle \in\left(X^{\prime} \cup X_{0}\right)$ $\times Y_{0}$ with $\beta<\alpha$ by (7.20) and (7.15). For such $\langle\alpha, \beta\rangle$, we have $\tilde{\tau}(\alpha, \beta)=$ $\tau(\tilde{\phi}(\alpha), \tilde{\psi}(\beta))$ by (7.20). Suppose that $\tilde{\tau}(\alpha, \beta)=0$. Then, since $\tau$ satisfies (7.13), we should have $\tilde{\phi}(\alpha) \leq \tilde{\psi}(\beta)$. By $(7.19)$, we have $\beta>\tilde{\psi}(\beta)+\xi+\theta$. On the other hand, by (7.17), $\alpha=\tilde{\phi}^{2}(\alpha) \leq \tilde{\phi}(\alpha)+\xi+\theta$. It follows that

$$
\alpha \leq \tilde{\phi}(\alpha)+\xi+\theta \leq \tilde{\psi}(\beta)+\xi+\theta<\beta .
$$

This is a contradiction. - (Lemma 7.3)

A quartet $p=\left\langle X^{p}, Y^{p}, \tau^{p},\left\langle\phi_{\xi}^{p}, \psi_{\xi}^{p}: \xi \in D^{p}\right\rangle\right\rangle$ (not necessarily an element of $\left.\mathbb{P}_{0}\right)$ with $D^{p} \in\left[\omega_{2}\right]^{\aleph_{0}}$ is said to be an extendable condition if $\left\langle X^{p}, Y^{p}, \tau^{p}, \phi_{\xi}^{p}, \psi_{\xi}^{p}\right\rangle$ is a $\xi$-extendable semi-condition for all $\xi \in D^{p}$.

For extendable conditions $p, q$ with

$$
p=\left\langle X^{p}, Y^{p}, \tau^{p},\left\langle\phi_{\xi}^{p}, \psi_{\xi}^{p}: \xi \in D^{p}\right\rangle\right\rangle, \quad q=\left\langle X^{q}, Y^{q}, \tau^{q},\left\langle\phi_{\xi}^{q}, \psi_{\xi}^{q}: \xi \in D^{q}\right\rangle\right\rangle,
$$

we write $p \leq_{1} q$ if

$$
\begin{aligned}
& X^{p} \supseteq X^{q}, \quad Y^{p} \supseteq Y^{q}, \quad \tau^{p} \supseteq \tau^{q}, \quad D^{p} \supseteq D^{q}, \\
& \phi_{\xi}^{p} \supseteq \phi_{\xi}^{q}, \psi_{\xi}^{p} \supseteq \psi_{\xi}^{q} \quad \text { for all } \xi \in D^{p} ; \\
& \tau^{p} \uparrow\left(X^{p} \backslash X^{q}\right) \times Y^{q} \equiv 1 .
\end{aligned}
$$

Note that for $p, q \in \mathbb{P}_{0}$, we have $p \leq_{1} q$ if and only if $p \leq_{\mathbb{P}_{0}} q$.

Lemma 7.4 (Extension lemma). Suppose that

$$
p=\left\langle X^{p}, Y^{p}, \tau^{p},\left\langle\phi_{\xi}^{p}, \psi_{\xi}^{p}: \xi \in D^{p}\right\rangle\right\rangle
$$

is an extendable condition for some $D^{p} \in\left[\omega_{2}\right]^{\aleph_{0}}$. Then there is a $q \in \mathbb{P}_{0}$ with $q=\left\langle X^{q}, Y^{q}, \tau^{q},\left\langle\phi_{\xi}^{q}, \psi_{\xi}^{q}: \xi \in D^{q}\right\rangle\right\rangle$ such that $D^{q}=D^{p}$ and $q \leq_{1} p$.

Furthermore, if $p_{0} \in \mathbb{P}_{0}$ is such that $p \leq_{1} p_{0}$ then $q \leq_{\mathbb{P}_{0}} p_{0}$.

Proof. The second part of the lemma is clear once the condition $q$ as in the claim of the lemma is found, since (7.8)(e) holds for such $q$ and $p_{0}$ being the relation $\leq_{1}$ is easily seen to be transitive.

To construct the desired $q \in \mathbb{P}_{0}$, let $\left\langle\xi_{n}: n \in \omega\right\rangle$ be an enumeration of $D^{p}$ such that each $\xi \in D^{p}$ appears infinitely often in the enumeration. First, construct $\left\langle X_{n}, Y_{n}, \tau_{n},\left\langle\phi_{\xi, n}, \psi_{\xi, n}: \xi \in D^{p}\right\rangle\right\rangle, n \in \omega$, recursively such that

$$
\left\langle X_{0}, Y_{0}, \tau_{0},\left\langle\phi_{\xi, 0}, \psi_{\xi, 0}: \xi \in D^{p}\right\rangle\right\rangle=p
$$


(7.24) $\left\langle X_{n+1}, Y_{n+1}, \tau_{n+1}, \phi_{\xi_{n}, n+1}, \psi_{\xi_{n}, n+1}\right\rangle$ is the $\xi_{n}$-extendable semi-condition constructed as in Lemma 7.3 from the $\xi_{n}$-extendable semicondition $\left\langle X_{n}, Y_{n}, \tau_{n}, \phi_{\xi_{n}, n}, \psi_{\xi_{n}, n}\right\rangle$.

(7.25) $\phi_{\xi, n+1}=\phi_{\xi, n}$ and $\psi_{\xi, n+1}=\psi_{\xi, n}$ for all $\xi \in D^{p}$ with $\xi \neq \xi_{n}$.

Along with the recursive construction above, it can be shown easily that $\left\langle X_{n}, Y_{n}, \tau_{n}, \phi_{\xi, n}, \psi_{\xi, n}\right\rangle$ is a $\xi$-extendable semi-condition for all $n \in \omega$ and $\xi \in D^{p}$. Hence the construction in (7.24) is actually possible at each step.

Let

$$
\begin{aligned}
& X^{q}=\bigcup_{n \in \omega} X_{n}, \quad Y^{q}=\bigcup_{n \in \omega} X_{n}, \quad \tau^{q}=\bigcup_{n \in \omega} \tau_{n}, \\
& D^{q}=D^{p}, \quad \phi_{\xi}^{q}=\bigcup_{n \in \omega} \phi_{\xi, n}, \quad \psi_{\xi}^{q}=\bigcup_{n \in \omega} \psi_{\xi, n} \quad \text { for all } \xi \in D^{q} .
\end{aligned}
$$

For all $\xi \in D^{q}$, there are infinitely many $n \in \omega$ such that $\xi_{n}=\xi$. For such $n, \phi_{\xi, n}$ is an involution on $X_{n}$ and $\psi_{\xi, n}$ is an involution on $Y_{n}$. It follows that $\phi_{\xi}^{q}$ is an involution on $X^{q}$ and $\psi_{\xi}^{q}$ is an involution on $Y^{q}$. Hence $q=\left\langle X^{q}, Y^{q}, \tau^{q},\left\langle\phi_{\xi}^{q}, \psi_{\xi}^{q}: \xi \in D^{q}\right\rangle\right\rangle$ is a condition in $\mathbb{P}_{0}$. Also we have

$$
\tau^{q} \uparrow\left(X^{q} \backslash X^{p}\right) \times Y^{p}=\bigcup_{n \in \omega} \tau^{q} \uparrow\left(X_{n+1} \backslash X_{n}\right) \times Y^{p} \equiv 1 .
$$

Thus, this $q$ is as desired.

(Lemma 7.4)

Lemma 7.5. (CH) $\mathbb{P}_{0}$ satisfies the $\aleph_{2}$-c.c.

Proof. Actually we shall show that $\mathbb{P}_{0}$ satisfies a strong form of $\aleph_{2^{-}}$ Knaster property.

Suppose $p^{\zeta} \in \mathbb{P}_{0}$ with $p^{\zeta}=\left\langle X^{\zeta}, Y^{\zeta}, \tau^{\zeta},\left\langle\phi_{\xi}^{\zeta}, \psi_{\xi}^{\zeta}: \xi \in D^{\zeta}\right\rangle\right\rangle$ for $\zeta \in \omega_{2}$. By the $\Delta$-system lemma (Theorem 4.1) and the pigeon-hole principle, there are a stationary $S \subseteq \omega_{2}, X, Y, D \in\left[\omega_{2}\right]^{\aleph_{0}}, \tau: X \times Y \rightarrow 2$ and $\phi_{\xi}: X \rightarrow X$, $\psi_{\xi}: Y \rightarrow Y$ for $\xi \in D$ such that

(7.26) $X^{\zeta}, \zeta \in S$, form a $\Delta$-system with root $X$, and $Y^{\zeta}, \zeta \in S$, form a $\Delta$-system with root $Y$;

(7.27) $\quad \tau^{\zeta}\lceil X \times Y=\tau$ for all $\zeta \in S$;

(7.28) $D^{\zeta}, \zeta \in S$, form a $\Delta$-system with root $D$;

(7.29) $\quad \phi_{\xi}^{\zeta} \uparrow X=\phi_{\xi}$ and $\psi_{\xi}^{\zeta} \uparrow Y=\psi_{\xi}$ for all $\zeta \in S$ and $\xi \in D$;

(7.30) $\tau^{\zeta} \uparrow\left(X^{\zeta} \backslash X\right) \times Y \equiv 1$ for all $\zeta \in S$.

Note that (7.27) is possible since, by $\mathrm{CH}$, there are at most $\left|{ }^{X \times Y} 2\right| \leq 2^{\aleph_{0}}=$ $\aleph_{1}<\aleph_{2}$ possible values of $\tau^{\zeta}\lceil X \times Y$. (7.29) is possible since, by $\mathrm{CH},(7.4)$ and countability of $D$, there are at most $\aleph_{1}$ possible values of $\left\langle\phi_{\xi}^{\zeta}: \xi \in D\right\rangle$ 
and $\left\langle\psi_{\xi}^{\zeta}: \xi \in D\right\rangle$. (7.30) is possible by (7.7) and since we can choose $S$ such that $\min \left(X^{\zeta} \backslash X\right)>\sup (Y)$ for all $\zeta \in S$.

Now suppose $\zeta, \eta \in S$ with $\zeta<\eta$. We show that $p^{\zeta}$ and $p^{\eta}$ are compatible. Let $X^{p}=X^{\zeta} \cup X^{\eta}, Y^{p}=Y^{\zeta} \cup Y^{\eta}$ and $D^{p}=D^{\zeta} \cup D^{\eta}$. For $\xi \in D^{p}$, let $\phi_{\xi}^{p}: X^{p} \rightarrow X^{p}$ and $\psi_{\xi}^{p}: Y^{p} \rightarrow Y^{p}$ be defined by

$$
\phi_{\xi}^{p}=\left\{\begin{array}{ll}
\phi_{\xi}^{\zeta} & \text { if } \xi \in D^{\zeta} \backslash D, \\
\phi_{\xi}^{\zeta} \cup \phi_{\xi}^{\eta} & \text { if } \xi \in D, \\
\phi_{\xi}^{\eta} & \text { otherwise, }
\end{array} \quad \psi_{\xi}^{p}= \begin{cases}\psi_{\xi}^{\zeta} & \text { if } \xi \in D^{\zeta} \backslash D, \\
\psi_{\xi}^{\zeta} \cup \psi_{\xi}^{\eta} & \text { if } \xi \in D, \\
\psi_{\xi}^{\eta} & \text { otherwise. }\end{cases}\right.
$$

Finally, let $\tau^{p}: X^{p} \times Y^{p} \rightarrow 2$ be such that

$$
\tau^{p}(\alpha, \beta)= \begin{cases}\tau^{\zeta}(\alpha, \beta) & \text { if }\langle\alpha, \beta\rangle \in X^{\zeta} \times Y^{\zeta} \\ \tau^{\eta}(\alpha, \beta) & \text { else if }\langle\alpha, \beta\rangle \in X^{\eta} \times Y^{\eta} \\ 1 & \text { otherwise, }\end{cases}
$$

for all $\langle\alpha, \beta\rangle \in X^{p} \times Y^{p}$. It is easy to see that $p=\left\langle X^{p}, Y^{p}, \tau^{p},\left\langle\phi_{\xi}^{p}, \psi_{\xi}^{p}\right.\right.$ : $\left.\left.\xi \in D^{p}\right\rangle\right\rangle$ is an extendable condition and $p \leq_{1} p^{\zeta}, p^{\eta}$. In particular, (7.22) holds for $p \leq_{1} p^{\zeta}$ and $p \leq_{1} p^{\eta}$ because of (7.30) and the "otherwise" clause of (7.32). By the extension lemma (Lemma 7.4), there is a $q \in \mathbb{P}_{0}$ with $q \leq_{1} p$. Hence, by the second half of the lemma, it follows that $q \leq_{\mathbb{P}_{0}}$ $p^{\zeta}, p^{\eta}$. (Lemma 7.5)

A modification of the $\Delta$-system argument in the proof of Lemma 7.5 is also used to prove the following:

Lemma 7.6. (CH) $\mathbb{P}_{0}$ forces (6.5).

Proof. We show that $\mathbb{P}_{0}$ forces the following:

(7.33) For any stationary $S \subseteq E_{\omega_{1}}^{\omega_{2}}$ and $A_{\zeta}, B_{\zeta} \in\left[\omega_{2}\right]^{\aleph_{0}}$ for $\zeta \in S$, there is a stationary $T \subseteq S$ such that for any $n \in \omega$ and pairwise distinct $\zeta_{i}, \eta_{i} \in T, \quad i \in n$, there is $\xi<\omega_{2}$ such that $\dot{\phi}_{\xi}{ }^{\prime \prime} A_{\zeta_{i}}=A_{\eta_{i}}$ and $\dot{\psi}_{\xi}{ }^{\prime \prime} B_{\zeta_{i}}=B_{\eta_{i}}$ for all $i \in n$.

Note that, by $\sigma$-closedness and $\aleph_{2}$-c.c. of $\mathbb{P}_{0}$ (proved in Lemma 7.5), $\omega_{1}$ and $\omega_{2}$ in generic extensions by $\mathbb{P}_{0}$ remain $\omega_{1}$ and $\omega_{2}$.

Suppose that $\dot{S}$ is a $\mathbb{P}_{0}$-name of a stationary subset of $E_{\omega_{1}}^{\omega_{2}}$. Let $\left\langle\dot{A}_{\zeta}\right.$ : $\zeta \in \dot{S}\rangle$ and $\left\langle\dot{B}_{\zeta}: \zeta \in \dot{S}\right\rangle$ be $\mathbb{P}_{0}$-names of sequences of countable subsets of $\omega_{2}$. Let

$$
\tilde{S}=\left\{\zeta \in E_{\omega_{1}}^{\omega_{2}}: \|_{\mathbb{P}_{0}} " \zeta \notin \dot{S} "\right\}
$$

Then $\models_{\mathbb{P}_{0}}$ " $\dot{S} \subseteq \tilde{S}$ " and hence $\tilde{S}$ is a stationary subset of $E_{\omega_{1}}^{\omega_{2}}$.

Since $\mathbb{P}_{0}$ is $\sigma$-closed, we can find $p_{\zeta} \in \mathbb{P}_{0}$ and $A_{\zeta}, B_{\zeta} \in\left[\omega_{2}\right]^{\aleph_{0}}$ such that

$$
p_{\zeta}=\left\langle X^{\zeta}, Y^{\zeta}, \tau^{\zeta},\left\langle\phi_{\xi}^{\zeta}, \psi_{\xi}^{\zeta}: \xi \in D^{\zeta}\right\rangle\right\rangle,
$$




$$
p_{\zeta} \Vdash_{\mathbb{P}_{0}} " \zeta \in \dot{S}, \dot{A}_{\zeta}=A_{\zeta} \text { and } \dot{B}_{\zeta}=B_{\zeta} "
$$

for all $\zeta \in \tilde{S}$. Without loss of generality, we may assume that

$$
A_{\zeta} \subseteq X^{\zeta} \quad \text { and } \quad B_{\zeta} \subseteq Y^{\zeta} .
$$

By the $\Delta$-system lemma (Theorem 4.1) and the pigeon-hole principle, there are a stationary $\tilde{S}_{0} \subseteq \tilde{S}, X, Y, D \in\left[\omega_{2}\right]^{\aleph_{0}}, \tau: X \times Y \rightarrow 2$ and $\phi_{\xi}: X \rightarrow X$, $\psi_{\xi}: Y \rightarrow Y$ for $\xi \in D$ such that

(7.37) $\quad X^{\zeta}, \zeta \in \tilde{S}_{0}$, form a $\Delta$-system with $\operatorname{root} X$, and $Y^{\zeta}, \zeta \in \tilde{S}_{0}$, form a $\Delta$-system with $\operatorname{root} Y$;

$$
\begin{aligned}
& \sup (Y)<\min \left(X^{\zeta} \backslash X\right) \text { for all } \zeta \in \tilde{S}_{0} \\
& \tau^{\zeta} \uparrow X \times Y=\tau \text { for all } \zeta \in \tilde{S}_{0} ; \\
& D^{\zeta}, \zeta \in \tilde{S}_{0}, \text { form a } \Delta \text {-system with root } D ; \\
& \phi_{\xi}^{\zeta} \uparrow X=\phi_{\xi} \text { and } \psi_{\xi}^{\zeta} \uparrow Y=\psi_{\xi} \text { for all } \zeta \in \tilde{S}_{0} \text { and } \xi \in D ; \\
& \tau^{\zeta} \uparrow\left(X^{\zeta} \backslash X\right) \times Y \equiv 1 \text { for all } \zeta \in \tilde{S}_{0} \text { (this follows from (7.38) and (7.7)); } \\
& X^{\zeta}, \zeta \in \tilde{S}_{0}, \text { are order-isomorphic and } Y^{\zeta}, \zeta \in \tilde{S}_{0} \text {, are order-isomorphic; }
\end{aligned}
$$

Note that the order-isomorphisms of $X^{\zeta}$ 's and $Y^{\zeta}$ 's do not move elements of $X$ and $Y$, respectively.

(7.44) The order-isomorphism sending $X^{\zeta}$ to $X^{\eta}$ sends $\tau^{\zeta} \uparrow\left(\left(X^{\zeta} \backslash X\right) \times Y\right)$ to $\tau^{\eta} \uparrow\left(\left(X^{\eta} \backslash X\right) \times Y\right)$, while the order-isomorphism sending $Y^{\zeta}$ to $Y^{\eta}$ sends $\tau^{\zeta} \uparrow\left(X \times\left(Y^{\zeta} \backslash Y\right)\right)$ to $\tau^{\eta} \uparrow\left(X \times\left(Y^{\eta} \backslash Y\right)\right)$. These order-

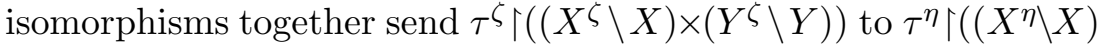
$\left.\times\left(Y^{\eta} \backslash Y\right)\right)$;

(7.45) The order-isomorphism sending $X^{\zeta}$ to $X^{\eta}$ sends $A_{\zeta}$ to $A_{\eta}$, and the order-isomorphism sending $Y^{\zeta}$ to $Y^{\eta}$ sends $B_{\zeta}$ to $B_{\eta}$.

Note that $\bar{p}=\left\langle X, Y, \tau,\left\langle\phi_{\xi}, \psi_{\xi}: \xi \in D\right\rangle\right\rangle$ is a condition in $\mathbb{P}_{0}$ and $p_{\zeta} \leq \mathbb{P}_{0} \bar{p}$ for all $\zeta \in \tilde{S}_{0}$ (the condition (7.8)(e) for $\bar{p}$ and $p_{\zeta}$ holds by (7.42)).

Let $\dot{T}$ be a $\mathbb{P}_{0}$-name such that

$$
\|_{\mathbb{P}_{0}} " \dot{T}=\left\{\zeta \in \tilde{S}_{0}: p_{\zeta} \in \dot{G}\right\} "
$$

where $\dot{G}$ is the standard $\mathbb{P}_{0}$-name of the generic set.

Claim 7.6.1. $\bar{p} \vdash_{\mathbb{P}_{0}}$ " $\dot{T}$ is a stationary subset of $\omega_{2}$ ".

$\vdash$ Since $\mathbb{P}_{0}$ satisfies the $\aleph_{2}$-c.c. by Lemma 7.5 , for any $\mathbb{P}_{0}$-name $\dot{C}$ of a club subset of $\omega_{2}$, there is a club subset $C$ of $\omega_{2}$ (in the ground model) such

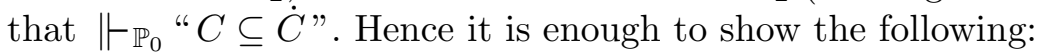

(7.47) For any $q \leq_{\mathbb{P}_{0}} \bar{p}$ and any club subset $C$ of $\omega_{2}$, there are $p \leq_{\mathbb{P}_{0}} q$ and $\zeta \in C \cap \tilde{S}_{0}$ such that $p \leq_{\mathbb{P}_{0}} p_{\zeta}$. 
To show (7.47), let $q=\left\langle X^{q}, Y^{q}, \tau^{q},\left\langle\phi_{\xi}^{q}, \psi_{\xi}^{q}: \xi \in D^{q}\right\rangle\right\rangle$ and let $\zeta \in C \cap \tilde{S}_{0}$ be such that

$$
\left(X^{\zeta} \backslash X\right) \cap X^{q}=\emptyset, \quad\left(Y^{\zeta} \backslash Y\right) \cap Y^{q}=\emptyset \quad \text { and } \quad\left(D^{\zeta} \backslash D\right) \cap D^{q}=\emptyset .
$$

This is possible by (7.37) and since $C \cap \tilde{S}_{0}$ is stationary.

Let $X^{*}=X^{q} \cup X^{\zeta}, Y^{*}=Y^{q} \cup Y^{\zeta}, D^{*}=D^{q} \cup D^{\zeta}$. For $\xi \in D^{*}$, let $\phi_{\xi}^{*}$ and $\psi_{\xi}^{*}$ be partial functions from $X^{*}$ to $X^{*}$ and from $Y^{*}$ to $Y^{*}$ respectively defined by

$$
\phi_{\xi}^{*}=\left\{\begin{array}{ll}
\phi_{\xi}^{q} & \text { if } \xi \in D^{q} \backslash D, \\
\phi_{\xi}^{q} \cup \phi_{\xi}^{\zeta} & \text { if } \xi \in D, \\
\phi_{\xi}^{\zeta} & \text { otherwise }
\end{array} \quad \psi_{\xi}^{*}= \begin{cases}\psi^{q} & \text { if } \xi \in D^{q} \backslash D \\
\psi_{\xi}^{q} \cup \psi_{\xi}^{\zeta} & \text { if } \xi \in D, \\
\psi_{\xi}^{\zeta} & \text { otherwise. }\end{cases}\right.
$$

Finally, let $\tau^{*}: X^{*} \times Y^{*} \rightarrow 2$ be defined by

(7.50) $\tau^{*}(\alpha, \beta)= \begin{cases}\tau^{q}(\alpha, \beta) & \text { if }\langle\alpha, \beta\rangle \in X^{q} \times Y^{q}, \\ \tau^{\zeta}(\alpha, \beta) & \text { else if }\langle\alpha, \beta\rangle \in X^{\zeta} \times Y^{\zeta}, \\ 1 & \text { otherwise, }\end{cases}$

for $\langle\alpha, \beta\rangle \in X^{*} \times Y^{*}$. Then $p^{*}=\left\langle X^{*}, Y^{*}, \tau^{*},\left\langle\phi_{\xi}^{*}, \psi_{\xi}^{*}: \xi \in D^{*}\right\rangle\right\rangle$ is an extendable condition and we have $p^{*} \leq_{1} q, p_{\zeta}:(7.22)$ for $p^{*}$ and $p_{\zeta}$ follows from

$$
\tau^{*} \uparrow\left(\left(X^{*} \backslash X^{\zeta}\right) \times Y^{\zeta}\right)=\tau^{*} \uparrow\left(\left(X^{q} \backslash X\right) \times\left(Y^{\zeta} \backslash Y\right)\right) \cup \tau^{*} \uparrow\left(\left(X^{q} \backslash X\right) \times Y\right) \equiv 1
$$

where we have $\tau^{*} \uparrow\left(\left(X^{q} \backslash X\right) \times\left(Y^{\zeta} \backslash Y\right)\right) \equiv 1$ by the definition (7.50) of $\tau^{*}$ and $\tau^{*} \uparrow\left(\left(X^{q} \backslash X\right) \times Y\right) \equiv 1$ by $q \leq_{\mathbb{P}_{0}} \bar{p}$ (in particular, by the condition $(7.8)(\mathrm{e})$ in the definition of $\left.\leq_{\mathbb{P}_{0}}\right)$.

By the extension lemma (Lemma 7.4) it follows that there is $p \in \mathbb{P}_{0}$ with $p \leq_{1} p^{*}$ and hence $p \leq_{\mathbb{P}_{0}} q, p_{\zeta} . \dashv($ Claim 7.6.1)

Claim 7.6.2. $\bar{p}$ forces that $\dot{T}$ is as in (7.33) for $\left\langle\dot{A}_{\zeta}: \zeta \in \dot{S}\right\rangle$ and $\left\langle\dot{B}_{\zeta}\right.$ : $\zeta \in \dot{S}\rangle$.

$\vdash$ By Claim 7.6.1 it is enough to prove the following:

(7.51) For any $q \leq_{\mathbb{P}_{0}} \bar{p}$ and $n \in \omega$, if $\zeta_{i}, \eta_{i} \in \tilde{S}_{0}$ are pairwise distinct and $q \Vdash_{\mathbb{P}_{0}}$ " $\zeta_{i}, \eta_{i} \in \dot{T}$ for $i \in n$ ", then there is $p \leq_{\mathbb{P}_{0}} q$ with

$$
p=\left\langle X^{p}, Y^{p}, \tau^{p},\left\langle\phi_{\xi}^{p}, \psi_{\xi}^{p}: \xi \in D^{p}\right\rangle\right\rangle
$$

and $\xi_{0} \in D^{p}$ such that $\phi_{\xi_{0}}^{p} \mid X^{\zeta_{i}}: X^{\zeta_{i}} \rightarrow X^{\eta_{i}}$ and $\psi_{\xi_{0}}^{p}\left\lceil Y^{\zeta_{i}}: Y^{\zeta_{i}} \rightarrow\right.$ $Y^{\eta_{i}}$ are order-isomorphisms for all $i<n$.

Without loss of generality, we may assume that

$$
q \leq_{\mathbb{P}_{0}} p_{\zeta_{i}}, p_{\eta_{i}} \quad \text { for all } i<n .
$$


Let

$$
q=\left\langle X^{q}, Y^{q}, \tau^{q},\left\langle\phi_{\xi}^{q}, \psi_{\xi}^{q}: \xi \in D^{q}\right\rangle\right\rangle .
$$

Take $\xi_{0} \in \omega_{2} \backslash\left(D^{q} \cup \sup \left(X^{q}\right) \cup \sup \left(Y^{q}\right)\right)$ and let $D^{*}=D^{q} \cup\left\{\xi_{0}\right\}$. Let $X^{*}=$ $X^{q}, Y^{*}=Y^{q}$ and $\tau^{*}=\tau^{q}$. Let $\phi_{\xi_{0}}^{*}: \bigcup_{i<n} X^{\zeta_{i}} \cup \bigcup_{i<n} X^{\eta_{i}} \rightarrow \bigcup_{i<n} X^{\zeta_{i}} \cup$ $\bigcup_{i<n} X^{\eta_{i}}$ be the involution sending $X^{\zeta_{i}}$ order-isomorphically to $X^{\eta_{i}}$ and vice versa for all $i<n$ and $\psi_{\xi_{0}}^{*}: \bigcup_{i<n} Y^{\zeta_{i}} \cup \bigcup_{i<n} Y^{\eta_{i}} \rightarrow \bigcup_{i<n} Y^{\zeta_{i}} \cup \bigcup_{i<n} Y^{\eta_{i}}$ be the involution sending $Y^{\zeta_{i}}$ order-isomorphically to $Y^{\eta_{i}}$ and vice versa for all $i<n$. Let $\phi_{\xi}^{*}=\phi_{\xi}^{q}$ and $\psi_{\xi}^{*}=\psi_{\xi}^{q}$ for $\xi \in D^{q}$.

Then $p^{*}=\left\langle X^{*}, Y^{*}, \tau^{*},\left\langle\phi_{\xi}^{*}, \psi_{\xi}^{*}: \xi \in D^{*}\right\rangle\right\rangle$ is an extendable condition with $p^{*} \leq_{1} q$. To see this, we have to check $\left\langle X^{*}, Y^{*}, \tau^{*}, \phi_{\xi_{0}}^{*}, \psi_{\xi_{0}}^{*}\right\rangle$ satisfies (7.12) and (7.14). But this follows from (7.42), (7.52) and (7.45).

By the Extension Lemma (Lemma 7.4) there is $p \in \mathbb{P}_{0}$ with $p \leq_{1} p^{*}$. Clearly $p$ forces that $\xi_{0}$ as above satisfies (7.33) together with $\dot{T},\left\langle\dot{A}_{\zeta}: \zeta \in \dot{S}\right\rangle$ and $\left\langle\dot{B}_{\zeta}: \zeta \in \dot{S}\right\rangle . \dashv($ Claim 7.6.2)

Since the argument above can be repeated below arbitrary element of $\mathbb{P}_{0}$, it follows that $\mathbb{P}_{0}$ forces (7.33). - (Lemma 7.6)

\section{A summary of consistency results and some open problems.} The following is a summary of consistency results in connection with the combinatorial principles in Fig. 5 where (1)-(7) below correspond to the separation lines (1)-(7) drawn in Fig. 9.

(1): By adding random reals. More precisely, start from a model $V$ of $\mathrm{CH}$ and force with (the positive elements of) the measure algebra $\mathbb{B}$ of, say, Maharam type $\aleph_{2}$. $\mathbb{B}$ can be seen as a (measure-theoretic) product of random forcing and thus inherits some of the homogeneity property of finite support product. This is used to prove $\mathfrak{d} \mathfrak{o}=\aleph_{1}$ in the generic extension. It is also well-known that the ground model functions from $\omega$ to $\omega$ dominate the functions in a generic extension by a measure algebra. Hence we have $\mathfrak{d}=\aleph_{1}$ in the model. K. Kunen proved that there is a $\kappa$-Lusin gap for an uncountable $\kappa$ in such a model. On the other hand, I. Juhász, L. Soukup and Z. Szentmiklóssy proved in [13] that there is no $\aleph_{2}$-Lusin gap under $\mathrm{C}^{\mathrm{S}}\left(\aleph_{2}\right)$. This proves that $\mathrm{C}^{\mathrm{S}}\left(\aleph_{2}\right)$ does not hold in the generic extension.

This observation may also be interpreted as pinning down of the difference in the extent of homogeneity of product forcing and the forcing by measure-theoretic products in terms of whether the principle $\mathrm{C}^{\mathrm{s}}\left(\aleph_{2}\right)$ holds.

(2): A model constructed by J. Brendle and T. LaBerge in [1] realizes this separation.

(3): By the model in Theorem 3.8 of I. Juhász and K. Kunen [12] in which $\mathrm{C}^{\mathrm{S}}\left(\aleph_{2}\right)$ and $\mathfrak{d o}>\aleph_{1}$ hold. The model is obtained by a finite support 
product of $\aleph_{2}$ posets of cardinality $\aleph_{1}$ starting from a model of CH. From this, it follows easily that $\mathfrak{b}^{*}=\aleph_{1}$ and $\mathfrak{d}=\aleph_{2}$.

(4): By adding Cohen reals. More exactly, start from a model $V$ of $\mathrm{CH}$ and then add, say, $\aleph_{2}$ Cohen reals (by $\operatorname{Fn}\left(\aleph_{2}, 2\right)$ ). Then by Corollary $4.8(\mathrm{c}$ ) we have $\operatorname{IP}\left(\aleph_{2}, \aleph_{1}\right)$ in the generic extension. Just as in (3), we have $\mathfrak{d}=\aleph_{2}$ in such a generic extension and it is shown in S. Fuchino, S. Koppelberg and S. Shelah [8] that WFN holds there.

(5): By a model of Hechler.

(6): By Theorem 6.4.

(7): By Corollary 4.9.

(8): By Theorem 5.2. See [11] for the proof of $\Vdash_{\mathbb{S}^{\kappa}} " \neg \mathrm{WFN".}$

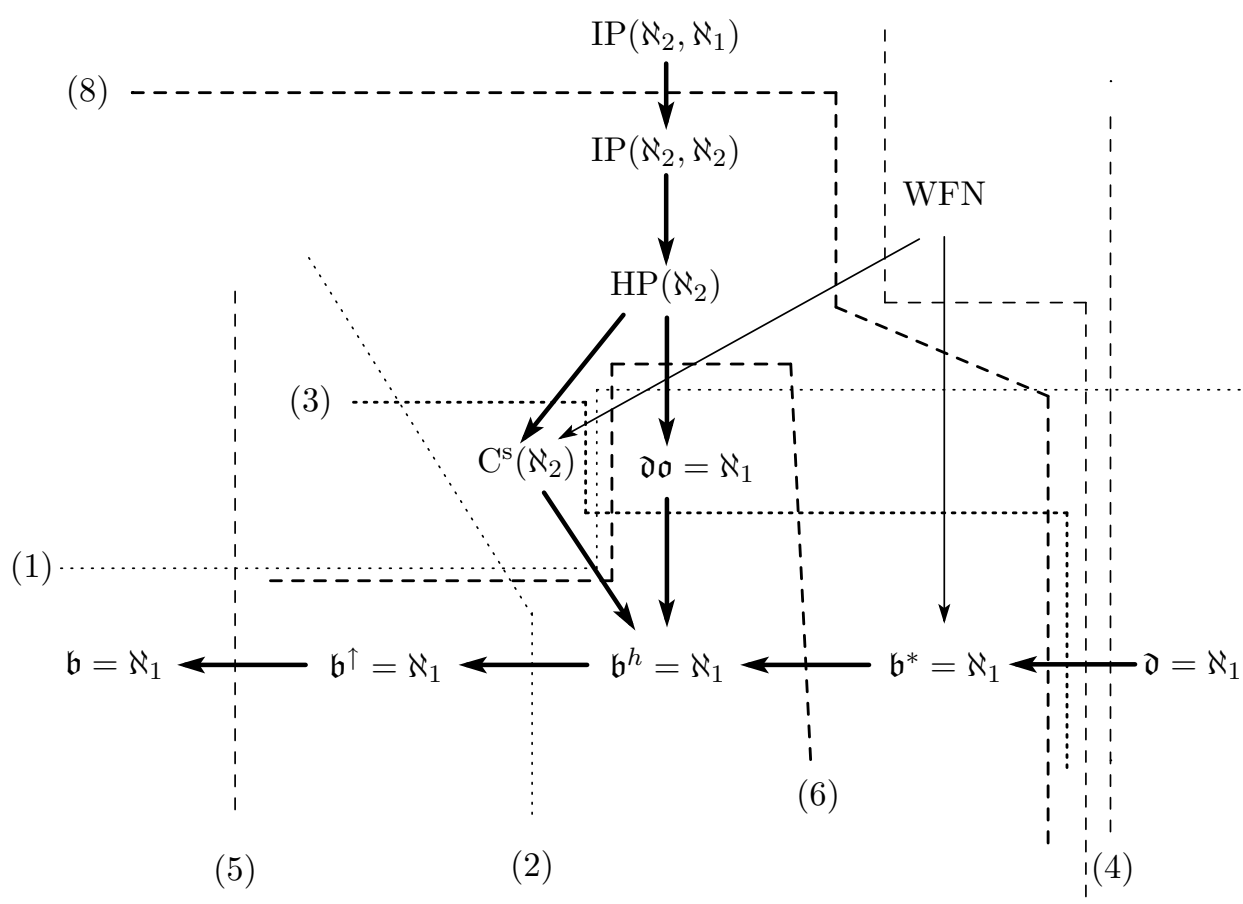

Fig. 9

Finally, we shall mention some open problems. In [6] it is shown that $\mathfrak{a}=\aleph_{1}$ follows from WFN where $\mathfrak{a}$ is the almost disjoint number. In [4], it is then shown that, under some additional assumptions, $\mathfrak{a}=\aleph_{1}$ already follows from SEP which is a weakening of WFN. Therefore, it seems natural to ask the following question:

Problem 1. Does $\mathfrak{a}=\aleph_{1}$ follow from $\operatorname{HP}\left(\aleph_{2}\right)$ or $\operatorname{IP}\left(\aleph_{2}, \lambda\right)$ for $\lambda=$ $\aleph_{1}, \aleph_{2}$ ? 
Problem 2. Does WFN imply $\operatorname{HP}\left(\aleph_{2}\right)$ or $\mathfrak{d} \mathfrak{o}=\aleph_{1}$ ?

The model of $\mathfrak{b}^{*}=\aleph_{2}$ and $\mathfrak{d} \mathfrak{o}=\aleph_{1}$ satisfies a strong form of negation of $\mathrm{C}^{\mathrm{S}}\left(\aleph_{2}\right)$. This suggests the following problem:

Problem 3. Does $\operatorname{HP}\left(\aleph_{2}\right)$ (or even $\mathrm{C}^{\mathrm{S}}\left(\aleph_{2}\right)$ ) imply $\mathfrak{b}^{*}=\aleph_{1}$ ?

At the moment, we do not have any model separating $\operatorname{HP}(\kappa)$ and $\operatorname{IP}(\kappa, \kappa)$ for $\kappa>\omega_{1}$.

Problem 4. Is $\operatorname{HP}(\kappa)+\neg \operatorname{IP}(\kappa, \kappa)$ consistent for some (or any) $\kappa>\aleph_{1}$ ?

In Corollary 4.9 which realizes the separation (7) in Fig. 9, a very strong large cardinal property is assumed.

Problem 5. Can we construct a model realizing (7) in Fig. 9 starting from ZFC without any large cardinal?

The property (A) used in the proof of Theorem 6.4 and proved to be consistent with $\mathrm{CH}$ in Section 7 seems to be of independent interest.

Problem 6. Is $\neg(\mathrm{A})$ consistent with $\mathrm{ZFC}+\mathrm{CH}$ (or even with $\mathrm{ZFC}+$ $\mathrm{GCH})$ ?

\section{References}

[1] J. Brendle and T. LaBerge, Forcing tightness in products of fans, Fund. Math. 150 (1996), 211-226.

[2] K. Ciesielski and J. Pawlikowski, The Covering Property Axiom, CPA, Cambridge Univ. Press, 2004.

[3] K. Eda, M. Kada and Y. Yuasa, The tightness about sequential fans and combinatorial properties, J. Math. Soc. Japan 49 (1997), 181-197.

[4] S. Fuchino and S. Geschke, Some combinatorial principles defined in terms of elementary submodels, Fund. Math. 181 (2004), 233-255.

[5] S. Fuchino, S. Geschke, S. Shelah and L. Soukup, On the weak Freese-Nation property of complete Boolean algebras, Ann. Pure Appl. Logic 110 (2001), 89-105.

[6] S. Fuchino, S. Geschke and L. Soukup, On the weak Freese-Nation property of $\mathcal{P}(\omega)$, Arch. Math. Logic 40 (2001), 425-435.

[7] - - - - Principles capturing features of generic extensions by almost side-by-side product, in preparation.

[8] S. Fuchino, S. Koppelberg and S. Shelah, Partial orderings with the weak FreeseNation property, Ann. Pure Appl. Logic 80 (1996), 35-54.

[9] S. Fuchino, S. Shelah and L. Soukup, Sticks and clubs, ibid. 90 (1997), 57-77.

[10] S. Fuchino and S. Soukup, More set-theory around the weak Freese-Nation property, Fund. Math. 154 (1997), 159-176.

[11] S. Geschke, On $\sigma$-filtered Boolean algebras, Dissertation, Freie Univ. Berlin, 1999.

[12] I. Juhász and K. Kunen, The power set of $\omega$, elementary submodels and weakenings of CH, Fund. Math. 170 (2001), 257-265.

[13] I. Juhász, L. Soukup and Z. Szentmiklóssy, Combinatorial principles from adding Cohen reals, in: Logic Colloquium '95 (Haifa), Lecture Notes in Logic 11, Springer, 1998, 79-103. 
[14] M. Kada and Y. Yuasa, Cardinal invariants about shrinkability of unbounded sets, Topology Appl. 74 (1996), 215-223.

[15] K. Kunen, Set Theory, North-Holland, 1980.

[16] A. Miller, Infinite combinatorics and definability, Ann. Pure Appl. Logic 41 (1989), 179-203.

[17] S. Shelah, an unpublished note for I. Juhász, 2002.

Department of Computer Science

and Systems Engineering

Graduate School of Engineering

Kobe University

Rokko-dai 1-1

Nada, Kobe 657-8501, Japan

E-mail: brendle@kurt.scitec.kobe-u.ac.jp
Department of Natural Science and Mathematics College of Engineering, Chubu University Kasugai, Aichi 487-8501, Japan E-mail: fuchino@isc.chubu.ac.jp

Received 23 March 2007;

in revised form 16 July 2007 